\title{
Annual Meeting of the Royal Belgian Society of Laboratory Medicine: “Women's health: from puberty to menopause"
}

\author{
Brussels, Belgium \\ November 19, 2021 \\ Congress President \\ Prof. Dr. Etienne Cavalier \\ Scientific Committee \\ Etienne Cavalier \\ Matthijs Oyaert \\ Michel Langlois \\ Pieter Vermeersch \\ Joris Delanghe
}

\section{Organizing Committee}

Xavier Bossuyt

Etienne Cavalier

Frederic Cotton

Anne Demulder

Nathalie De Vos

Joris Delanghe

Damien Gruson

Beatrice Gulbis

Michel Langlois

Caroline Legoff

Laurence Lutteri

Hugo Neels

Matthijs Oyaert

Florent Vanstapel

Martine Vercammen

Pieter Vermeersch

Alain Verstraete

Ilse Weets

Fleur Wolff

${ }^{*}$ These abstracts have been reproduced directly from the material supplied by the authors, without editorial alteration by the staff of this Journal. Insufficiencies of preparation, grammar, spelling, style, syntax, and usage are the authors. 


\title{
SP1
}

\section{HORMONES DURING MENSTRUAL CYCLE AND OVARIAN STIMULATION}

\author{
$\underline{\text { O. Goldrat }}^{1}$
}

${ }^{1}$ Fertility Clinic, Department of Obstetrics and Gynecology, CUB-hôpital Erasme, ULB, Brussels, Belgium

During the menstrual cycle, follicular recruitment occurs at early follicular phase, followed by dominancy of usually one follicle that reaches ovulation. This process is sequential and involves a 2cells- 2 gonadotropins mechanism.

In IVF, controlled ovarian stimulation (COS) is done, followed by artificial ovulation triggering, allowing to recruit several mature oocytes to be fertilized. Obtained embryos can be transferred to achieve pregnancy and surplus embryos are frozen for future use.

Follicular and hormonal monitoring are performed before and after egg retrieval and allow to depict any variations that might modify the course of the cycle. These include, among others, cases with OHSS risk, low estradiol level or increased progesterone level during follicular phase. Correct management of patients depends on analysis of all parameters in these situations.

\section{SP2}

\section{ORAL CONTRACEPTIVES AND THROMBOSIS}

\author{
J. Douxfils ${ }^{1,2,3}$
}

${ }^{1}$ Namur Thrombosis and Hemostasis Center, Namur, Belgium, ${ }^{2}$ Namur Research Institute for Life Sciences, Namur, Belgium, ${ }^{3}$ Department of Pharmacy, Faculty of Medicine, University of Namur, Namur, Belgium

Combined oral contraceptives (COCs) induce several changes in the levels of coagulation factors. Procoagulant factors are often increased, while anticoagulant factors are decreased. These effects are more pronounced with COC containing ethinylestradiol in association with desogestrel, gestodene, drospirenone, dienogest or cyproterone acetate compared with $\mathrm{COC}$ containing ethinylestradiol in combination with levonorgestrel. More recently, estradiol and estetrol has been used to replace ethinylestradiol and demonstrated a better hemostatic profile compared to ethinylestradiol.

Venous thromboembolism (VTE) risk increases when multiple risk factors, including genetic and environmental, are present simultaneously. COC use causes changes in coagulation that modify the prothrombotic state induced by preexisting hemostatic alterations in a supra-additive manner. Therefore, testing appears to be of importance not only before implementing COC but also to monitor any potential thrombogenicity induced by COC therapy. Inherited genetic factors, such as factor V Leiden, G20210A prothrombin mutation, antithrombin, protein C or protein S deficiencies, non-O blood group, as well as CYP2C9 2 and the rs 4379368 mutations, have all been identified as genetic predictive risk factors of VTE in women.

Specific testing such as the endogenous thrombin potential based activated protein $\mathrm{C}$ resistance could permit the assessment of the thrombogenicity induced by hormonal therapies and inherited or acquired thrombophilia. 


\title{
SP3
}

\section{EPIDEMIOLOGICAL CHA(LLE/N)GES WITHIN HERPESVIRUSES DURING PUBERTY AND CHILD-BEARING AGE}

\author{
$\underline{\text { E. Padalko }}^{1}$ \\ ${ }^{1}$ Laboratory Medicine Department, Ghent University Hospital, Ghent, Belgium
}

The main objective of the current presentation is to describe the epidemiological evolutions and to highlight analytical challenges for serological laboratory tests for herpesviruses.

Within herpesviruses the accent in the current presentation will be given to epidemiology and related laboratory challenges within Herpes Simplex Viruses (HSV). There are clear trends with at the moment still not fully understood reasons for in decreasing HSV-1 seroprevalence in adolescents in various high income countries. HSV seroprevalence studies normally use type-specific serological assays: this approach is error prone and in particular from the sensitivity point of view as type-specific glycoprotein $\mathrm{G}$ assays are in the rule $+/-85 \%$ sensitive for $\mathrm{HSV}-2$ and even less for HSV-1. Lower HSV seroprevalence in the child-bearing age is putting young pregnant women at higher risk for primary HSV during pregnancy and by its turn also for neonatal herpetic infections. Following changing epidemiological trends within genital herpes as well as laboratory analytical challenges, caution in interpreting the results is needed.

Next to the epidemiological trends in HSV seroprevalence, also epidemiology of other relevant during pregnancy herpesviruses as Varicella-Zoster Virus (VZV) and Cytomegalovirus will be discussed.

\section{References}

Balaeva T. et al. Seroprevalence and correlates of herpes simplex virus type 2 infection among young adults in Arkhangelsk, Northwest Russia: a population-based cross-sectional study. BMC Infect Dis 2016 Oct 28;16(1):616.

Korr G. et al. Decreasing seroprevalence of herpes simplex virus type 1 and type 2 in Germany leaves many people susceptible to genital infection: time to raise awareness and enhance control. BMC Infect Dis 2017 Jul 6;17(1):471.

Woestenberg PJ et al. Herpes simplex virus type 1 and type 2 in the Netherlands: seroprevalence, risk factors and changes during a 12-year period. BMC Infect Dis. 2016 Aug 2;16:364.

Yousuf W. et al. Herpes simplex virus type 1 in Europe: systematic review, meta-analyses and meta-regressions. BMJ Glob Health 2020 Jul;5(7):e002388.

\section{SP4}

\section{THE FEMALE UROGENITAL AND GUT MICROBIOME: KEY PLAYERS IN HEALTH AND FERTILITY ACROSS LIFE}

\section{$\underline{\text { T. Demuyser }}{ }^{1,2}$}

${ }^{1}$ Department of Microbiology and Infection Control, UZ Brussel, Brussels, Belgium, ${ }^{2}$ Center for Neurosciences, Vrije Universiteit Brussel, Brussels, Belgium

Micro-organisms can be found virtually anywhere, including in and on the human body. Throughout life, you are host to a dynamic ecosystem of microbes, your so-called microbiota. Bacteria and other micro-organisms interact with certain crucial physiological functions. In my talk, I will introduce you to these microscopic companions that are of major importance through a woman's lifetime.

From conception onwards, hormones in a mother's circulation interact with her microbiota, preparing the microbial niches of the genital tract for pregnancy and childbirth. At birth, neonates are primed with their mother's distinct vaginal 
and faecal microbiota, jump-starting their immune system. As such, the vagina is an underappreciated organ that is a lot more than just a passageway for neonates. It can affect the health of women and their offspring for an extent that only recently is acknowledged.

Post-partum, the neonate's microbiota undergo a rapid change, guided by the rich and nutritious properties of the mother's breast milk. Packed with pre- and probiotic compounds, antibodies, and leucocytes, the colostrum is a natural "super-kefir", acting as a kind of immune system transplant.

From toddler years onwards, the microbiota ripen and adapt to outside signals, dietary changes etc. During childhood and adolescence, hormonal changes, life stressors, and dietary habits can have a lasting impact on mental and physical well-being. At this point in a woman's lifespan, apart from the maturation of the microbiotagut-brain axis, the evolution in the urogenital microbiota becomes crucial in her reproductive cycle. Upon increased oestrogen and progesterone exposure, the genital microbial niche shifts towards a community mainly including lactobacilli.

Throughout life, microbial communities are dynamic and very susceptible to potential beneficial or harmful factors. An imbalance in the composition of the vaginal microbiota, called dysbiosis, may result in bacterial or fungal vaginosis. In postmenopausal women however, a "physiological dysbiosis" occurs, with a decrease in lactobacilli. Vaginal microbiota remain crucial at this moment in life, because they can exert profound effects on the development of vulvovaginal atrophy, vaginal dryness, and impaired sexual health.

\section{SP6}

\section{PRE-ECLAMPSIA RISK ASSESSMENT BY SFLT1/PLGF TESTING}

\section{J. Van Keirsbilck ${ }^{1}$}

${ }^{1}$ Department of Gynaecology, AZ Sint Jan, Brugge, Belgium

Pre-eclampsia (PE) is a leading cause of maternal and fetal/neonatal morbidity and mortality. The clinical diagnosis is generally based on the presence of hypertension and signs of maternal organ dysfunction. The clinical presentation and course of $\mathrm{PE}$ is variable, ranging from mild to severe and from early-onset PE, less than 34 weeks gestational age (GA), to late-onset PE at term. Pre-eclampsia may also be associated with intrauterine growth restriction (IUGR), causing neonatal morbidity and mortality. The prediction of the development of pre-eclampsia and the related adverse outcomes has always been poor. Early detection, on the other hand, enables optimal fetal and maternal management, and so reducing overall morbidity.

The literature demonstrated that disturbances in the angiogenic factors soluble fms-like tyrosine kinase receptor-1 (sFlt-1) and placental growth factor (PIGF) are implicated in the pathogenesis of PE and therefore can be of additional value in its diagnosis. In this regard, other placenta-related disorders like IUGR of stillbirth are also involved.

First trimester screening of pre-eclampsia by combining maternal factors, maternal blood pressure, uterine artery dopplers and PlGF measurement is highly accurate, especially for early PE. Prevention of PE can be enabled by using aspirin (150mg) prophylaxis in the high-risk group.

After 24 weeks GA, an increased sFlt-1/PIGF ratio, can not only be used to detect pre-eclampsia, but also those who are at high risk for developing the disease. Establishing a high-risk group makes close follow up and monitoring more efficient and cost-effective. Repeated sFlt-1/PlGF measurements can also be helpful in predicting its course.

So the use of sFlt-1, PlGF or the sFlt-1/PlGF ratio can be an additional tool in the prediction, diagnosis of PE in the first and second trimester and therefore in the overall management of PE. 


\title{
SP7
}

\section{CLINICAL VALUE AND INTERPRETATION OF AMH DURING REPRODUCTIVE YEARS}

\author{
$\underline{\text { A. Delbaere }^{1}}$ \\ ${ }^{1}$ Fertility Clinic, Department of Obstetrics and Gynecology, Hôpital Erasme, Université Libre de Bruxelles, Brussels, \\ Belgium
}

Anti-Müllerian Hormone (AMH) formerly known as Müllerian inhibiting substance is a homodimeric glycoprotein belonging to the Transforming Growth Factor ß superfamily and mainly secreted by the gonads. AMH expression is sexually differentiated and plays a key role in sexual differentiation as its abundant production by the fetal testis leads to irreversible Müllerian duct regression. Besides its classical role in male sexual differentiation, AMH secretion has been detected in the fetal ovary as of mid-gestation and is produced by the granulosa cells of pre-antral and antral follicles. AMH is implicated in the initial recruitment of primordial follicles and modulates the follicular sensitivity to FSH. In women, AMH levels peak in young adulthood and decline over time until menopause. AMH is rather stable across the menstrual cycle although a slight peak has been reported in the mid-follicular phase with a drop at the beginning of the luteal phase. In clinics, AMH has been used as a marker of treatment efficacy and tumor progression in granulosa cells tumors. Serum AMH has been proposed as a marker of ovulatory dysfunction in patients with polycystic ovarian syndrome (PCOS). However, due to heterogeneity between studies, it is not recommended yet as a diagnostic test of PCOS. Over the last decades, AMH has emerged as a key marker of the ovarian reserve. Its serum levels are strongly correlated with the antral follicle count and are used in routine to individualize the ovarian stimulation treatment in assisted reproductive techniques. High AMH levels have been correlated to a high oocyte yield and a higher risk of developing an ovarian hyperstimulation syndrome. AMH level is however a poor predictor of fecundability and live birth rates. As a marker of the ovarian reserve, AMH has been used to counsel candidates for fertility preservation in situations at risk of premature ovarian insufficiency and for longitudinal follow-up of the ovarian function of young female cancer survivors. Although AMH levels have been related to age at menopause, they are not sufficiently indicative for long-term predictions of the reproductive lifespan.

\section{SP8}

\section{CLINICAL UTILITY OF BIOMARKERS IN THE DIAGNOSIS OF OVARIAN CANCER}

\section{$\underline{\text { D. Timmerman }}^{1}$}

${ }^{1}$ Department of Gynaecology, University Hospitals KU Leuven, Leuven, Belgium

The Trans-IOTA study is a prospective multicenter study on patients presenting with ovarian tumours.

\section{Main objective}

To investigate which proteins have added diagnostic value over demographic and ultrasound variables (from ADNEX model) to distinguish between benign vs. malignant tumours

\section{Secondary objectives}

To investigate which proteins play a role in the differentiation between different types of ovarian masses and to investigate which proteins may have added diagnostic value over demographic and ultrasound variables (from ADNEX model) to distinguish between different subtypes

\section{Tertiary objectives}

To assess the diagnostic accuracy of serum CA125 and HE4

\section{Results}

data from 932 patients have been analysed with univariable and multivariable analaysis. 


\title{
C1
}

\section{INTERNAL QUALITY CONTROL OF SERUM INDICES: FRESH OF FROZEN?}

\author{
M. Olbrecht ${ }^{1}$, A. van Dalem², F. Winnock ${ }^{1}$, M. Martin², P. Heyvaert ${ }^{2}$, T. Cluysen², I. Weets ${ }^{2}$ \\ ${ }^{1}$ Algemeen Stedelijk Ziekenhuis (ASZ), Aalst, Belgium, ${ }^{2}$ Vrije Universiteit Brussel (VUB), Universitair Ziekenhuis Brussel \\ (UZ Brussel), Department of Clinical Biology, Brussels, Belgium

\section{Objective}

The presence of hemolysis (H), icterus (I) and lipemia (L) in diagnostic samples are important preanalytical interferences. While the testing of the HIL indices is important for sample quality in routine practice in most clinical laboratories, there are no clear practical guidelines about the quality assurance of these serum indices.

The Biorad Liquichek Serum Indices control is intended to monitor and provide an objective assessment of the instrument's response in detecting hemolyzed, icteric or lipemic samples. With this study, we evaluate 2 internal quality control (IQC) protocols for HIL indices, using fresh or frozen aliquots in 2 different laboratories.

\section{Material and Methods}

To evaluate the difference between frozen aliquots and fresh non-frozen aliquots we did a 1-month evaluation. At the start, we aliquoted the original control bottle in 60 capped vials and stored them at $-20^{\circ} \mathrm{C}$, at the time of measurement the vial was thawed $1 \mathrm{~h}$ before use. For the fresh non-frozen control, the original bottle was thawed on Monday and stored refrigerated for one week of measurements. In total, we measured 6 indices (Hemolysis vial $\mathrm{H}$ index, Icterus vial I index, Lipemia vial L index, Non-Interfered vial H index, Non-Interfered vial I index and Non-Interfered vial L index) twice a day during 1 month on one 502 and four c702 modules (Roche Cobas 8000 ) allocated to 2 hospital sites (ASZ Aalst and UZ Brussel). We evaluated the difference for the 6 indices on each module between frozen aliquots and fresh non-frozen aliquots with a paired t-test.

\section{Results}

In $76 \%$ the $p$-value of the paired t-test is less than 0.05 , there is a statistically significant difference between the use of the frozen and fresh aliquots. Nevertheless, the difference in means between the frozen and fresh aliquots was always less than 2SD, even more in $88 \%$ the difference in means was even less than $1 \mathrm{SD}$.

\section{Conclusion}

An IQC protocol for HIL indices using the Biorad Liquichek Serum Indices as based on fresh non-frozen aliquots or frozen aliquots can both be used.

\section{C2}

\section{OVERDOSE OF EMTRICITABINE/ELVITEGRAVIR/TENOFOVIR ALAFENAMIDE/COBICISTAT IN TWO AUTO-INTOXICATIONS}

Olivier Mortelele ${ }^{\dagger}, 1, \star$, Celine Gys ${ }^{\dagger, 2}$, Babette Van Rafelghem ${ }^{3}$, Eleanor Berry ${ }^{2}$, Alexander L. N. van Nuijs ${ }^{2}$, Willy Lambert ${ }^{4}$, Werner Jacobs ${ }^{3}$, Sofie Schouwers ${ }^{1}$, Ellen Van Hemeldonck ${ }^{5}$, Luc Heytens ${ }^{5}$, Anissa Meskal ${ }^{1}$, Hugo Neels ${ }^{2}$, Adrian Covaci ${ }^{2}$

${ }^{\dagger}$ Joint first authors

${ }^{1}$ Laboratorium for Clinical Biology, GZA Sint-Augustinus, Oosterveldlaan 24, 2610 Antwerp, Belgium, ${ }^{2}$ Toxicological Center, University of Antwerp, Universiteitsplein 1, 2610 Antwerp, Belgium, ${ }^{3}$ Department of Forensic Medicine, Antwerp 
University Hospital, University of Antwerp, Edegem, Belgium, ${ }^{4}$ Laboratory of Toxicology, Department of Bioanalysis, Faculty of Pharmaceutical Sciences, Ghent University, Ottergemsesteenweg 460, 9000 Ghent, Belgium, ${ }^{5}$ Department of Intensive Care, GZA Sint-Augustinus, Oosterveldlaan 24, 2610 Antwerp, Belgium

*corresponding author: olivier.mortele@gmail.com

\title{
Objectives
}

Data on overdose with HIV-inhibitors are scarce $(1,2)$, and reports including measurements of the levels of these compounds are inexistent. We present a case report of two independent auto-intoxications by self-administered overdose with the combination drug emtricitabine/elvitegravir/tenofovir alafenamide/cobicistat (F/E/TAF/C).

\section{Material and methods}

Case 1 involves a 40-year-old HIV seropositive male who stated to have voluntarily taken 60 tablets of $200 \mathrm{mg} \mathrm{F} / 150 \mathrm{mg}$ $\mathrm{E} / 10 \mathrm{mg} \mathrm{TAF} / 150 \mathrm{mg}$ C. Case 2 concerns an 18-year-old male who stated to have voluntarily ingested 30 tablets of the same formulation during the night. Emtricitabine was quantified in seven serum samples obtained during hospitalization (case 1) and post-mortem serum and urine (case 2) respectively (3).

\section{Results}

The measured emtricitabine serum concentrations (case $1: 17.6 \mathrm{mg} / \mathrm{L}$, case 2: $14.4 \mathrm{mg} / \mathrm{L}$, therapeutic range: 0.1 to $3.0 \mathrm{mg} / \mathrm{L}$ (4)), confirmed the self-declared overdosages. Both patients presented with a loss of consciousness, hypotensive crisis, metabolic acidosis and increased hepatic transaminases upon admission at the emergency department. Furthermore, a reversible moderate renal impairment during hospitalization was observed in case 1 . The patient was discharged from the hospital after 7 days. Minutes after admission; patient 2 developed a junctional heart rhythm of 40 beats/min without functional output. Advanced life support was performed without success, after which the patient died.

\section{Conclusions}

Two auto-intoxications with the HIV-inhibiting medication F/E/TAF/C resulted in different outcomes. For the first time, emtricitabine levels following overdose are reported.

\section{References}

1. Álvarez H et al. Infection. 2019;47(1):115-9.

2. Lee $\mathrm{M}$ et al. AIDS Res Ther. 2015;12:18.

3. Kromdijk W et al. Journal of chromatography B. 2013;919-920:43-51.

4. Baselt RC. Disposition of Toxic Drugs and Chemicals in Man. Eleventh ed. Seal Beach, CA, USA: Biomedical Publications; 2017.

\section{C3}

\section{THE FREE CORTISOL INDEX: CORRELATION WITH THE FREE FRACTION DETERMINED BY LIQUID CHROMATOGRAPHY-TANDEM MASS SPECTROMETRY AFTER EQUILIBRIUM DIALYSIS AND ESTABLISHMENT OF REFERENCE INTERVALS}

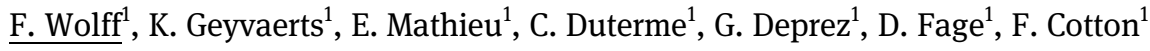 \\ ${ }^{1}$ LHUB-ULB, department of clinical chemistry, Brussel, Belgium

\section{Objectives}

Changes in cortisol binding globulin (CBG) impact the total serum cortisol concentration and affect the accurate assessment of adrenal function. Free biologically cortisol can be calculated using different equations or directly 
measured after complicated procedures. In this study, the correlation between the calculated free cortisol index (FCI) and the free fraction measured was assessed. Reference limits for FCI were then determined.

\title{
Material and methods
}

The FCI was first calculated for 45 healthy workers using the Bonte formula: [cortisol (nmol/L)]/[CBG (nmol/L)])x100 [1]. The CBG level was determined by a competitive radioimmunoassay (Diasource) and the total cortisol concentration, measured with an electrochemiluminescent assay (Cortisol Gen II, Roche Diagnostics). The correlation between FCI and the free fraction measured after equilibrium dialysis and liquid chromatography-tandem mass spectrometry (LC-MS/MS) was studied using the Spearman coefficient. Reference limits for FCI defined as the 2.5 and the 97.5 percentiles were established on 141 healthy hospital workers with serum samples collected between 7:30 am and $12 \mathrm{pm}$.

\section{Results}

The free cortisol index calculated with the Bonte formula on the 45 samples correlated significantly with the free cortisol measured with LC/MS-MS after equilibrium dialysis with a Spearman coefficient of $0.6328(\mathrm{p}<0.0001)$. Although the cortisol and CBG levels were statistically higher in women under contraceptive uses compared with women not taking them as well as men, the calculated FCI did not differ between these groups (Kruskall-Wallis test, $\mathrm{p}>0.05$ ). The median FCI (P2.5-P97.5) obtained for women (n: 94) and men (n:47) were respectively 21.5\% (10.7-44.5\%) and 24.8\% (13.8-38.3\%).

\section{Conclusion}

This study highlighted a significant correlation between the FCI and the free cortisol measured, it has also allowed the establishment of reference intervals for FCI on 141 healthy workers.

\section{Reference}

[1] Bonte HA, van den Hoven RJ, van der Sluijs Veer G, Vermes I. The use of free cortisol index for laboratory assessment of pituitary-adrenal function. Clin Chem Lab Med. 1999.

\section{C4}

\section{ON THE NATURE OF PECULIAR EXPECTORATED BRONCHIAL CASTS: CAN INFRARED SPECTROSCOPY ENLIGHTEN US?}

\author{
$\underline{\text { S. De Bruyne }}{ }^{1}$, D. Ommeslag ${ }^{2}$, J. Himpe ${ }^{3}$ J. Delanghe ${ }^{3}$, A.-M. van den Abeele ${ }^{1}$ \\ ${ }^{1}$ Department of Laboratory Medicine, AZ Sint-Lucas, Ghent, Belgium, ${ }^{2}$ Department of Pulmonary and Infectious Diseases, \\ AZ Sint-Lucas, Ghent, Belgium, ${ }^{3}$ Department of Diagnostic Sciences, Ghent University, Ghent, Belgium
}

Introduction

Bronchial casts can be defined as gelatinous structures originating from the airways. While several cases of bronchial cast formation have been reported in literature, unravelling its nature is often a difficult task. We applied for the first time infrared (IR) spectroscopy on a bronchial cast fragment originating from a patient who suffered from a 2-year history of frequent coughing accompanied by the occasional expectoration of viscous and thick white-yellow bronchial-like structures.

\section{Material and methods}

Analysis was performed using a Perkin Elmer Spectrum Two Attenuated total-reflectance Fourier transformed infrared (ATR-FTIR) spectrometer. Fatty acid chain length $\left(\mathrm{CH}_{3}\left(\mathrm{CH}_{2}\right)_{\mathrm{x}} \mathrm{COOH}\right)$ was calculated by taken the absorbance ratio of the $\mathrm{CH}_{2}$ symmetric stretching band at $2853 \mathrm{~cm}^{-1}$ and the $\mathrm{C}=\mathrm{O}$ stretching band from lipid esters at $1744 \mathrm{~cm}^{-1}$. 


\title{
Results
}

Intense bands were observed for $\mathrm{CH}_{2}$ asymmetric stretching vibrations at $2927 \mathrm{~cm}^{-1}$ and symmetric stretching vibrations at $2853 \mathrm{~cm}^{-1}$ originating from lipid chains. Furthermore, asymmetric $\mathrm{CH}_{3}$ stretching at $2954 \mathrm{~cm}^{-1}$ and symmetric stretching at $2866 \mathrm{~cm}^{-1}$ could be seen as shoulders in the spectrum. Moreover, a prominent peak corresponding to $\mathrm{C}=\mathrm{O}$ stretching band from lipid esters was found at $1744 \mathrm{~cm}^{-1}$. While amide I $\left(1640 \mathrm{~cm}^{-1}\right)$ and amide II $\left(1532 \mathrm{~cm}^{-1}\right)$ protein peaks are often the most abundant vibrational bands in the IR spectrum of a biological sample, the intensities in the spectrum of the bronchial cast were relatively low compared to the bands attributed to lipids, which indicated a high lipid to protein ratio. The mean acyl chain length was found to be 18 carbon atoms, suggesting the presence of longchain fatty acids ( $>$ C12). The markedly high lipid to protein ratio and presence of long-chain fatty acids derived from the bronchial cast's IR spectrum were very suggestive for chyloptysis. Taking into account the patient's prior history of radiation therapy for a lymphoma complicated by congestive heart failure, we hypothesized that an impairment of adequate lymphatic flow into the venous system due to a combination of lymphatic obstruction and high venous pressures was the most plausible culprit.

\section{Conclusion}

IR spectroscopy has the ability to provide the keys to unlock a longstanding medical mystery and should be considered as a valuable alternative tool in the exploration of a bronchial cast's nature.

\section{C5}

\section{NEW FAECAL CALPROTECTIN ASSAY BY IDS: VALIDATION AND COMPARISON TO DIASORIN METHOD}

\author{
$\underline{\text { V. Castiglione }}{ }^{1}$, M. Berodes ${ }^{1}$, P. Lukas ${ }^{1}$, E. Cavalier ${ }^{1}$, L. Lutteri ${ }^{1}$ \\ ${ }^{1}$ Department of Clinical Chemistry, University of Liège, CHU Sart-Tilman, 4000 Liège, Belgium, Liège, Belgium

\section{Background}

Faecal Calprotectin measurement is a non-invasive test used for the diagnosis and follow-up of inflammatory bowel disease (IBD), and its differentiation with irritable bowel symptom (IBS). IDS developed a new kit for stool extraction and Calprotectin measurement.

\section{Objective}

The aim of this study was to validate the IDS Calprotectin extraction device and immunoassay kit, and to compare it with the DiaSorin Liaison test in patients with known clinical status.

\section{Methods}

Precision was evaluated on 5 native samples measured 5 days in pentaplicate after extraction. Stability at $2-8^{\circ} \mathrm{C}$ and $-20^{\circ} \mathrm{C}$ were assessed for 7 days and 14 weeks, respectively. A lot to lot comparison was also performed. Finally, a total of 379 stool extracts were analysed with the IDS kit on iSYS, and compared with DiaSorin Liaison assay. Clinical data of these patients about IBD/IBS were collected.

\section{Results}

Intra and inter-assays CV ranged from $1.8 \%$ to $3.0 \%$, and $2.5 \%$ to $4.1 \%$ respectively, for the tested ranges (39.6 to 1545.8 $\mu \mathrm{g} / \mathrm{g}$ ). Samples could be stored for up to 4 days at $2-8^{\circ} \mathrm{C}$, and up to 4 weeks at $-20^{\circ} \mathrm{C}$. Lot to lot comparison showed a good correlation (Lot1 $=1,0$ Lot $2+31.8 ; p>0.05)$. The Passing and Bablok regression showed no significant deviation from 
linearity between the two methods (IDS $=1.06$ DiaSorin $-0.6 ; p>0.05)$. According to the recommended cut-offs $(<50 \mu \mathrm{g} /$ g:negative; 50-120:equivocal; >120:positive), the IDS assay identified more IBD and IBS patients than DiaSorin, which had more borderline results (17 vs 20\%, respectively). Similarly, using a $250 \mu \mathrm{g} / \mathrm{g}$ cut-off, widely used among physicians, the IDS method detected more patients with IBD.

\title{
Conclusion
}

The IDS fecal Calprotectin had good analytical validation parameters. Compared to the DiaSorin method, IDS calprotectin assay showed comparable results, but outperformed it in the identification of more IBD patients.

\section{Disclosures}

EC is an accountant for both IDS and Diasorin.

\section{C6}

\section{IMPACTS OF VARIOUS PRE-ANALYTICAL CONDITIONS ON NEUROLOGICAL BLOOD-BASED BIOMARKERS}

\author{
A. Ladang ${ }^{1}$, L. Rigaud ${ }^{1}$, G. Sqalli ${ }^{1}$, E. Cavalier ${ }^{1}$ \\ ${ }^{1}$ Clinical chemistry / CHU de Liège, Liège, Belgium

\section{Objectives}

New technologies allowing the measurement of neurological biomarkers in the blood are emerging. Pre-analytical conditions are known to have an impact on the dosage of amyloid- $\beta$ (A $\beta$ ) and total Tau (t-Tau) when measured in cerebrospinal fluid (CSF). Therefore, pre-analytical studies on blood-based measurement of A $\beta$ and t-Tau are required. In this study, we are assessing the impact of homogenization, delayed centrifugation and tube handling on plasma collected with three different EDTA tubes for $A \beta_{1-40}, A \beta_{1-42}$, ratio $A \beta_{1-42} / A \beta_{1-40}$ and $t-T a u$.

\section{Methods}

$A \beta_{1-42}, A \beta_{1-40}$ and $t$-Tau were measured on plasma with the $S i M o A^{\circ}$ technology following various pre-analytical treatment. Relative and absolute concentrations as well as $A \beta_{1-42} / A \beta_{1-40}$ ratio were calculated and an analytical coefficient of variation was defined.

\section{Results}

The intra-run coefficient of variation were $7.1 \%, 5.3 \%$ and $7.4 \%$ for $A \beta_{1-42}, A \beta_{1-40}$ and t-Tau, respectively. Unexpectedly, we found an increased $A \beta$ concentration in polyethylene (PET) collection tubes when samples were processed right away. Yet, these higher levels in PET collection tubes rapidly disappeared after 1 hour at room temperature. The observed effects were corrected by the use of $A \beta_{1-42} / A \beta_{1-40}$ ratio. When polypropylene (PP) collection tubes were used, the homogenization, delayed centrifugation or tube handling had no impact on $\mathrm{A} \beta$ and t-Tau concentrations.

\section{Conclusions}

This study highlights the importance of a standardized pre-analytical procedure. The use of an EDTA polypropylene tube seems the most appropriate for $A \beta$ blood dosage. In case the use of PP collection tubes cannot be ensured, $\mathrm{A} \beta_{1-42} / \mathrm{A} \beta_{1-40}$ ratio should be favored. 


\title{
C7
}

\section{NEUROFILAMENT LIGHT CHAIN CONCENTRATION IN SERUM OF AGING POPULATION}

\author{
$\underline{\text { A. Ladang }}{ }^{1}$, S. Kovacs ${ }^{1}$, L. Lengelé2, M. Locquet ${ }^{2}$, J.-Y. Reginster ${ }^{2}$, O. Bruyère ${ }^{2}$, E. Cavalier ${ }^{1}$ \\ ${ }^{1}$ Clinical chemistry / CHU de Liège, Liège, Belgium, ${ }^{2}$ WHO Collaborating Centre for Public Health Aspects of Musculo- \\ skeletal Health and Aging, Division of Public Health, Epidemiology and Health Economics, University of Liège, Belgium, \\ Liège, Belgium
}

Neurology blood-based biomarkers are now emerging at an impressive speed. Among them, neurofilament light chains (NF-L) are one of the most promising and studied blood-based neurological biomarkers. Indeed, NF-L expression is now recognized to be modified in a broad spectrum of neurological diseases and traumatic brain injuries. Because many neurological diseases are also aging diseases, it is now urgent to understand influencing factors of NF-L expression in normal aging population.

In this study, we are assessing NF-L concentration in sera of a large cohort of community-dwelling adults aged over 65 years old. We studied the link between NF-L and various physiological factors (age, sex, BMI, GFR,...) but also with selfreported comorbidities or life-style habits.

Importantly, we showed that NF-L concentrations in serum are tightly linked to cystatin C concentrations ( $\mathrm{r}=0.5007$, $\mathrm{p}<0.0001)$ and consequently to estimated glomerular filtration rate and renal function $(\mathrm{r}=-0.492 ; \mathrm{p}<0.0001)$. We also observed that NF-L expression is dependent on age and BMI but not sex in both univariate and multivariate analysis. Among the self-reported comorbidities, subjects having reported neurological disorders, cardiovascular diseases or history of fracture had higher NF-L concentrations in univariate analysis. In the multivariate model, only subjects with self-reported neurological disorders showed higher on NF-L concentrations. NF-L concentrations were also increased when mini-mental state examination (MMSE) was decreased below 26 points but not when geriatric depression score (GDS) was increased above 5 points in both univariate and multivariate analysis. According to these results, we established reference ranges by age categories for subjects with or without altered renal function and we showed that renal status has more impact on reference ranges than age.

Our data highlight that NF-L concentrations in aging population are not driven by increasing number of comorbidities or depression. Yet, as many other blood biomarkers, NF-L blood concentrations are dependent on a proper kidney function and NF-L interpretation in patients suffering from renal failure should be taken with caution.

\section{C8}

\section{EVALUATION OF PRE-ANALYTICAL STABILITY AND DETERMINATION OF A CUTOFF VALUE FOR UMBILICAL CORD TOTAL BILIRUBIN TO PREDICT SIGNIFICANT NEONATAL HYPERBILIRUBINEMIA}

\author{
L. Vandervore $^{1}$, K. Eeckhout ${ }^{1}$, S. Weekx ${ }^{1}$, K. Ver Elst ${ }^{1}$, A. Meskal ${ }^{1}$, K. Plaskie ${ }^{2}$, S. Vermeiren ${ }^{1}$ \\ ${ }^{1}$ Department of Laboratory Medicine/GZA hospitals, Wilrijk, Belgium, ${ }^{2}$ Department of Neonatology/GZA hospitals, \\ Wilrijk, Belgium
}

\section{Objective(s)}

Post-natal monitoring of total bilirubin is important to prevent clinical complications of neurotoxic hyperbilirubinemia. However, neonates without risk factors may be discharged before 72 hours when potential hyperbilirubinemia might still develop prior to planned follow-up. Especially during the COVID-19 pandemic, early discharge was seen more often, 
underlining the need for an early biomarker. Here, neonatal umbilical cord bilirubin (UCB) was studied to predict development of significant neonatal hyperbilirubinemia.

\title{
Material and Methods
}

Venous UCB was systematically gathered during 4 months at the maternity wards of GZA hospitals. For each case, two lithium heparin tubes were filled and total bilirubin was determined (Alinity c, Abbott, USA). 1003 neonates were included and patient data were retrospectively analyzed. Pre-analytical stability was evaluated between different subgroups. Predictive value of UCB was studied with ROC-analysis.

\section{Results}

UCB results were significantly decreased in the total cohort when measured in light-exposed (H1) versus dark (HD) lithium heparin $(\mathrm{P}=0.0258)$. Significant photolysis depended on average UCB concentration, with lower concentrations being more susceptible (a term, $\mathrm{P}=0.0224$; $\mathrm{ABO}$ compatible, $\mathrm{P}=0.0231$; DAT negative, $\mathrm{P}=0.0207$ ). However, light exposure only led to a mean degradation of $-0.1 \%$ and did not seem to affect predictive value significantly (AUC UCB H1=0.721 versus AUC UCB $\mathrm{HD}=0.726 ; \mathrm{P}=0.2542$ ). $\mathrm{UCB}$ proved to be a significantly better predictive marker of phototherapy than ABO-incompatibility (AUC $=0.597$ ) or DAT positivity (AUC $=0.568$ ). $\mathrm{UCB}$ screening cutoff was $1.6 \mathrm{mg} / \mathrm{dL}$ in dark conditions and $1.5 \mathrm{mg} / \mathrm{dL}$ when exposed to light. Post-test probability at these cutoffs were 11.4 and 11.0\%. Diagnostic cutoff was not altered by light-exposure $(2.6 \mathrm{mg} / \mathrm{dL}$ ) and post-test probabilities were $33.3 \%$ (H1) and $36.1 \%$ (HD). Prevalence of significant hyperbilirubinemia was $9.57 \%$ within our total cohort.

\section{Conclusions}

This study shows that UCB measurement could aid in the prediction of neonatal hyperbilirubinemia and should be included in standard post-natal monitoring guidelines. Although total bilirubin is known to be photo-sensitive, we showed that light exposure did not affect predictive value of UCB.

\section{C9}

\section{QUANTIFICATION OF 25-HYDROXYVITAMIN D3 ON DRIED BLOOD SPOT BY LC-MS/MS}

\author{
C. Le Goff ${ }^{1}$, A. Coeme ${ }^{1}$, S. Peeters ${ }^{1}$, E. Cavalier ${ }^{1}$ \\ ${ }^{1}$ Clinical Chemsitry Departement, University Hospital of Liege, Liege, Belgium
}

\section{Objectives}

Vitamin D determination is of great interest because of its many health benefits (bone, immune, muscle, cardiovascular...). However, its analytical determination is far from an easy task. In the dried blood spot (DBS), the blood sample is directly soaked on to a paper. After drying, it can be analyzed by liquid chromatography coupled to tandem mass spectrometry.

This technique of sampling by dried blood spot (DBS) has many advantages such as low blood volume requirement, transportation and storage without special treatment, better analytes stability, enhanced clinical cooperation for the clinical trials, make it a very good techniques for the future. The aim of our work was to develop a sensitive and efficient method to measure 25-hydroxyvitamin $\mathrm{D}_{3}$ (25OH VitD3) on DBS.

\section{Materials and Methods}

DBS are cut from the paper support $(6 \mathrm{~mm})$. Then, samples are derivatized by a Cookson reagent, the DMEQ-TAD and analyzed by liquid chromatography coupled with tandem mass spectrometry (LC-MS/MS) method ((QTrap 6500 (Sciex, USA) coupled with LC-30A Nexera UHPLC system (Shimadzu,Japan)). After method development, a validation was 
carried out as well as uncertainty measurement and a method comparison between serum 25OH VitD3 and DBS after correction by hematocrit.

\title{
Results
}

The calibration curve was linear with a maximum bias $8 \%$ and a maximum CV 6.1\% for the range 5.7-69.2 ng/mL. The intra-run precision (CV) was 6 to 10.3\% (range: $6-53.2 \mathrm{ng} / \mathrm{mL}$ ) and total-run precision (CV) was 1.7-5.8\%. The trueness showed a maximum bias of $8.3 \%$ for an acceptable trueness of $10.5 \%$ according the EFLM data base. LOQ was $2.45 \mathrm{ng} / \mathrm{mL}$. The uncertainty measurement was maximum: $10.7 \%$ for our lowest concentration $(6 \mathrm{ng} / \mathrm{mL})$. The comparison between the DBS and serum showed a regression curve: DBS=1.17+0.938.25ViTD serum.

\section{Conclusion}

Results of this study have led to the development of a very promising assay method. An excellent correlation between the serum assay conventionally used and this new assay method was observed indicating the potential of this technique.

\section{C10}

\section{NECESSITY OF HARMONIZATION OF TISSUE TRANSGLUTAMINASE IGA ANTIBODY TESTS TO ALIGN CLINICAL DECISION MAKING IN COELIAC DISEASE}

\author{
$\underline{\text { L. Nevejan }}{ }^{1}$, P. Dobbels ${ }^{2}$ X. Bossuyt ${ }^{3}$, L. van Hoovels ${ }^{4}$
}

${ }^{1}$ Deparment of Laboratory Medicine, OLV Hospital, Aalst, Belgium \& Department of Laboratory Medicine, University Hospitals Leuven, Leuven, Belgium, ${ }^{2}$ Department of Gastroenterology, OLV Hospital, Aalst, Belgium, Aalst, Belgium, ${ }^{3}$ Department of Laboratory Medicine, University Hospital Leuven, Leuven, Belgium \& Department of Microbiology, Immunology \& Transplantion, KU Leuven, Leuven, Belgium, 'Department of Laboratory Medicine, OLV Hospital, Aalst, Belgium \& Department of Microbiology, Immunology \& Transplantation, KU Leuven, Leuven, Belgium

\section{Introduction}

The usefulness of serologic assays in monitoring coeliac disease (CD) is less evidence-based. Our study aimed to investigate the antibody ( $\mathrm{Ab}$ ) kinetics of tissue transglutaminase (tTG) and deamidated gliadin peptides (DGP) assays from two different manufacturers.

\section{Materials and Methods}

Newly diagnosed CD patients presenting at the OLV Hospital Aalst from November 2016 to March 2020 were prospectively included. They consulted the gastroenterologist 0, 3, 6, 12 and 24 months post-gluten-free diet (GFD). Eight CD serological assays were performed: tTG IgA/IgG and DGP IgA/IgG from Thermo Fisher (EliA ${ }^{\mathrm{TM}}$ assays on Phadia 200; cut-off 7 EliA $\mathrm{U} / \mathrm{mL}$ ) and Werfen (QUANTA Flash ${ }^{\circledR}$ assays on BIO-FLASH®; cut-off $\left.20 \mathrm{CU}\right)$.

\section{Results}

Thirteen CD patients were enrolled (median age 41 years; 85\% female). The trends of kinetic profiles obtained for the corresponding serological assays of the two manufacturers were similar. Fully $(n=7 / 13)$ - and partially $(n=2 / 13)$ GFD compliant patients showed a rapid decline in all Ab titers. The strongest decline (76-80 fold decrease) was observed for tTG IgA, the weakest for tTG IgG (6-fold decrease). Non-compliant patients $(n=2 / 13)$ continued to have elevated tTG and DGP Ab titers. At $t=6$ months, significantly more patients had tTG IgA values below the upper limit of normal (ULN) with the Thermo Fisher assay compared to the Werfen assay (10/12 vs. 3/12; $p=0.005)$. After harmonization of the cut-offs for both tTG IgA assays at a predefined specificity of $100 \%$ (i.e. EliA ${ }^{\mathrm{TM}}$ assays $14 \mathrm{EliA} \mathrm{U/mL;} \mathrm{QUANTA} \mathrm{Flash}{ }^{\circledR}$ assays $\left.100 \mathrm{CU}\right)^{1}$, the clinical interpretation for both manufacturers entirely synchronized from $t=3$ months, with a mean time to serological normalization of 11.9 months for fully and partially compliant patients. 


\section{Conclusion}

Clinicians must be aware of the differences in interpretation of serological assays between manufacturers and should rely on cut-offs that are harmonized based on pre-defined specificity.

\section{References}

1. Bogaert, L. et al. Autoimmun. Rev. 19, 102513 (2020).

\section{C11}

\section{PRE-ANALYTICAL RECOMMENDATIONS AND REFERENCE VALUES FOR CIRCULATING CALPROTECTIN ARE SAMPLE TYPE AND ASSAY DEPENDENT}

$\underline{\text { L. Nevejan }^{1}, \text { M. Myelemans }}{ }^{1}$, B. Vander Cruyssen ${ }^{2}$, M. Stubbe ${ }^{2}$, S. van den Bremt ${ }^{3}$, L. Hofman $^{3}$, M. Infantino $^{4}$, M. Manfredi $^{4}$, X. Bossuyt ${ }^{5}$, L. van Hoovels ${ }^{6}$

${ }^{1}$ Department of Laboratory Medicine, OLV Hospital, Aalst, Belgium \& Department of Laboratory Medicine, University Hospital Leuven, Leuven, Belgium, ${ }^{2}$ Department of Rheumatology, OLV Hospital, Aalst, Belgium, ${ }^{3}$ Department of Laboratory Medicine, OLV Hospital, Aalst, Belgium, ${ }^{4}$ Immunology and Allergy Laboratory Unit, San Giovanni di Dio Hospital, Florence, Italy, ${ }^{5}$ Department of Laboratory Medicine, University Hospital Leuven, Leuven, Belgium \& Department of Microbiology, Immunology and Transplantation, KU Leuven, Leuven, Belgium, ${ }^{6}$ Department of Laboratory Medicine, OLV Hospital, Aalst, Belgium \& Department of Microbiology, Immunology and Transplantation, KU Leuven, Leuven, Belgium

\section{Introduction}

Circulating calprotectin (cCLP) is a promising biomarker for the evaluation of neutrophil-related inflammation. Our aim was to compare reference values and the effect of pre-analytical variables for cCLP between different sample types and assays.

\section{Materials and methods}

Reference values were determined in 100 healthy individuals. Pre-analytical variables were evaluated in two healthy individuals and two rheumatoid arthritis patients and covered sample type (serum-gel, heparin, EDTA and citrate plasma), pre-centrifugation time $(<2 \mathrm{~h}, 6 \mathrm{~h}, 24 \mathrm{~h})$, storage condition $\left(2-8^{\circ} \mathrm{C}, 18-25^{\circ} \mathrm{C}, 30^{\circ} \mathrm{C}\right)$ and storage time $(24 \mathrm{~h}, 72 \mathrm{~h}$, 7 days). cCLP measurements were performed with the EliA ${ }^{\text {TM }}$ Calprotectin 2 assay (Thermo Fisher), MPR8/14 ELISA (Bühlmann Laboratories), QUANTA Flash® Circulating Calprotectin CIA (Werfen) and DiaSorin Calprotectin assay (DiaSorin S.P.A).

\section{Results}

Reference values were sample type dependent with the highest 95\% upper reference limit in serum $(1.730-2.260 \mu \mathrm{g} / \mathrm{mL})$, followed by heparin (1.474-1.854 $\mu \mathrm{g} / \mathrm{mL})$, EDTA $(0.750-0.860 \mu \mathrm{g} / \mathrm{mL})$ and citrate $(0.757-1.513 \mu \mathrm{g} / \mathrm{mL})$. For the Bühlmann ELISA, cCLP concentrations in EDTA for most samples were lower than the limit of quantification. cCLP method comparison revealed a very good correlation (spearman rank $r=0.941-0.994$ ) for Thermo Fisher, Werfen and DiaSorin for all sample types. For Bühlmann ELISA, moderate (0.710-0.732) to bad correlations (0.438-0.597) were obtained with serum and heparin/EDTA/citrate samples, respectively. Pre-analytically, the same trends were observed for all matrices for the assay from Thermo Fisher, Werfen and DiaSorin: (i) serum tubes need to be centrifuged $<2$ hours, (ii) plasma tubes need to be centrifuged $<6$ hours, (iii) all sample types can be stored up to 7 days refrigerated and up to $24 \mathrm{~h}$ at room temperature. However, significant deviations in pre-analytics were found for the Bühlmann assay.

\section{Conclusion}

Reference values and pre-analytical recommendations for cCLP not only depend on the sample type, but also on the cCLP assay used. 


\title{
C12
}

\section{PROGNOSTIC VALUE OF CIRCULATING CALPROTECTIN LEVELS ON THE CLINICAL COURSE OF COVID-19 DIFFERS BETWEEN SERUM, HEPARIN, EDTA AND CITRATE SAMPLE TYPES}

\author{
$\underline{\text { L. Nevejan }}^{1}$, T. Strypens ${ }^{1}$, M. van Nieuwenhove ${ }^{2}$, A. Boel ${ }^{3}$, L. Cattoir ${ }^{3}$, P. Meeus ${ }^{3}$, N. De Neve ${ }^{2}$, L. van Hoovels ${ }^{4}$ \\ ${ }^{1}$ Department of Laboratory Medicine, OLV Hospital, Aalst, Belgium \& Department of Laboratory Medicine, University \\ Hospital Leuven, Leuven, Belgium, ${ }^{2}$ Department of Intensive Care, OLV Hospital, Aalst, Belgium, ${ }^{3}$ Department of Lab- \\ oratory Medicine, OLV Hospital, Aalst, Belgium, ${ }^{4}$ Department of Laboratory Medicine, OLV Hospital, Aalst, Belgium \& \\ Department of Microbiology, Immunology and Transplantation, KU Leuven, Leuven, Belgium
}

\section{Introduction}

Circulating calprotectin (cCLP) is proposed as a promising biomarker to predict the severity of pathogen-associated tissue damage in COVID-19. We aimed to investigate the prognostic value of cCLP in serum, heparin, EDTA and citrate plasma in comparison to other inflammatory parameters.

\section{Materials and Methods}

COVID-19 patients requiring hospitalization between November 2020 and May 2021 presenting at the OLV Hospital Aalst were included as study population. Two SARS-CoV-2 negative control populations were included: i) patients requiring intensive care unit (ICU) post cardiovascular surgery $(\mathrm{n}=20)$ and ii) patients requiring non-elective hospitalisation (nonICU) ( $n=20)$. Following prognostic outcomes were investigated: i) severe or critical disease presentation; ii) requiring ICU admission; iii) need for mechanical ventilation or extra corporeal membrane oxygenation (ECMO) and iv) death after 30 days. These were compared between cCLP (EliA ${ }^{\mathrm{TM}}$ Calprotectin 2 assay, Thermo Fisher), IL-6, CRP, LDH, procalcitonin, and the 4C-mortality score by AUROC analysis.

\section{Results}

136 COVID-19 patients were included, in whom cCLP levels were higher compared to the respective control populations. cCLP levels in serum and heparin were significantly higher than in EDTA or citrate. cCLP levels were significantly higher in COVID-19 patients with i) severe/critical illness ( $n=70)$, ii) ICU admission ( $n=66)$ and iii) need for mechanical ventilation/ECMO ( $\mathrm{n}=25)$, but iv) not in patients who deceased after 30 days $(\mathrm{n}=41)$. The highest discriminatory power (AUC [95\% CI]) was respectively obtained by i) CRP (0.835 [0.755-0.914]); ii) EDTA cCLP (0.780 [0.688-0.873]); iii) EDTA cCLP (0.842 [0.758-0.925]) and iv) the 4C-mortality score (0.713 [0.608-0.818]).

\section{Conclusion}

Measuring cCLP in COVID-19 patients helps the clinician to predict having severe or critical illness at presentation, the requirement of ICU admission and the need for mechanical ventilation or ECMO, but not to predict mortality after 30 days. The discriminatory power of EDTA and citrate plasma CLP levels often outperformed serum and heparin plasma CLP levels.

\section{C13}

\section{PROZONE EFFECT IN AUTOIMMUNITY: BE WARY OF ISLET CELL ANTIBODIES SCREENING DILUTION RECOMMENDED BY MANUFACTURERS}

\author{
$\underline{\text { V. Castiglione }}^{1}$, M. Pesser ${ }^{1}$, P. Watrin ${ }^{1}$, E. Cavalier ${ }^{1}$, L. Lutteri ${ }^{1}$ \\ ${ }^{1}$ Department of Clinical Chemistry, University of Liège, CHU Sart-Tilman, 4000 Liège, Belgium, Liège, Belgium

\section{Background}

Islet cells antibodies (ICA), tested by indirect immunofluorescence (IIF) on monkey pancreas, is a marker for Type 1 diabetes. However, the sensitivity of this method is weak, its results might be inconsistent with other assays 
(anti-GAD or anti-IA2 antibodies) and its recommended screening dilution varies according to references, leading to false negative results.

\title{
Objective
}

We sought to test different screening dilutions for ICA IIF testing, and to compare it with anti-GAD65kd method.

\section{Methods}

A total of 188 samples were prospectively collected during 15 months. All sera were tested for ICA positivity by IIF (Menarini, ICAb Primate Pancreas) at 1:5 dilution according to the manufacturer recommendations. In addition, the samples were also tested at 1:20, 1:50 and 1:100 dilutions to verify prozone (Hook) effect. Anti-GAD65kd was measured by ELISA (anti-GAD, IgG, Euroimmun) to confirm the results.

\section{Results}

Amongst the 188 samples, 33 (17.5\%) samples were positive with both ICA and anti-GAD tests. However, only 16 of them $(48,5 \%)$ were positive at the 1:5 screening dilution recommended by the manufacturer. The other 17 (51,5\%) sera showed negative ICA at the 1:5 screening dilution, while being positive with 1:100 (or sometimes at 1:20 or 1:50). This is due to a prozone effect at low dilution which is avoided at higher dilutions, and was confirmed by anti-GAD positivity. One hundred fifty-five samples (80\%) were negative with both anti-GAD and ICA at 1:5 dilution. Finally, four patients had positive anti-GAD antibodies but no positive ICA at any dilution, which is in accordance with previously published data.

\section{Conclusion}

We showed that the 1:5 screening dilution recommended by the manufacturer leads to high false negative rate. Prozone effect is a well-known drawback of assays using antibodies, however it is still underestimated. Hence, we recommend to use a 1:100 screening dilution for ICA IIF testing.

\section{C14}

\section{COMPARISON OF LUMIPULSE G1200 AND SIMOA TECHNOLOGY FOR THE QUANTIFICATION OF ALZHEIMER'S BIOMARKERS}

\author{
G. Sqalli ${ }^{1}$, S. Kovacs ${ }^{1}$, F. Watar ${ }^{1}$, L. Lutteri ${ }^{1}$, E. Cavalier ${ }^{1}$, A. Ladang ${ }^{1}$ \\ ${ }^{1}$ Departement of Clinical Chemistry, University of Liège, CHU Sart-Tilman, Liège, Belgium, Liege, Belgium
}

\section{BACKGROUND-AIM}

The exploration of Amyloid beta peptides and tau protein in cerebrospinal fluid (CSF) is a key element in the prognostic and diagnostic evaluation, particularly in Alzheimer's disease.

\section{METHODS}

In this study, we compared LUMIPULSE® G1200 from Fujirebio and SR-X with SimoA technology from Quanterix for the determination of A $\beta 1-42$, A $\beta 1-40$, P-tau181, and T-tau. For the former, the Lumipulse G " $\beta$-Amyloid 1-42", " $\beta$-Amyloid 140", "Total Tau", "Ptau 181"assays were used. For the second, the "Neurology 3-Plex A Advantage kit" and the "pTau-181 Advantage V2 kit" were used. In order to realize this comparison, $56 \mathrm{CSF}$ for which these analysis were prescribed, were systematically measured on both devices. 


\title{
RESULTS
}

For the A $\beta 1-40$, the Passing-Bablok regression equation gave SIMOA $=-756.6(95 \%$ confidence interval (95\% $\mathrm{CI})$ is $-1,723.6$ to -8.97$)+0.97$ (0.89 to 1.10) LUMIPULSE. For the A $\beta 1-42$, the Passing-Bablok regression equation gave SIMOA $=-59.9$ (95\%CI: -110.6 to -23.3$)+0.79$ (0.73 to 0.86) LUMIPULSE. Therefore, a systematic bias for A $\beta 1-40$ and a systematic and proportional bias for A $\beta 1-42$ were observed. For T-tau and the P-tau181, the Passing-Bablock gave respectively SIMOA $=10.0(95 \%$ CI: -5 to 26.8$)+0.23(0.18$ to 0.28$)$ LUMIPULSE and SIMOA $=-1.18(95 \%$ CI: -4.96 to 1.45$)+0.59$ LUMIPULSE ( 0.48 to 0.71 ). A proportional bias was observed for these two analyses.Interestingly, it was noticed that the ratio of median T-tau/P-tau181 concentrations of Simoa and Lumipulse was different (0.22, 0.13 respectively). Although the individual measurements may differ, the ratio should be similar. This suggests that both methods are measuring different isoforms or that SimoA technol ogy may not measure all the T-tau.

\section{CONCLUSION}

In conclusion, the methods are not interchangeable and papers on SimoA technology should provide reference values. Caution should be particularly applied to the measurement of T-tau and P-tau181, due to the discrepancy observed between the two devices. In the future, a standardization will likely be needed.

\section{C15}

\section{THE NEW 2020 ESC GUIDELINES FOR THE MANAGEMENT OF ACUTE CORONARY SYNDROMES IN PATIENTS WITHOUT PERSISTENT ST-SEGMENT ELEVATION: ARE THEY APPLICABLE IN A CLINICAL BIOLOGY EMERGENCY LAB?}

\author{
$\underline{G . ~ S q a l l i ~}^{1}$, M. Lamtiri Laarif ${ }^{1}$, L. Vranken ${ }^{1}$, R. Gadisseur ${ }^{1}$, C. Le Goff ${ }^{1}$, E. Cavalier ${ }^{1}$ \\ ${ }^{1}$ Departement of Clinical Chemistry, University of Liège, CHU Sart-Tilman, Liège, Belgium, Liege, Belgium
}

\section{BACKGROUND-AIM}

In the context of the clinical presentation of acute coronary syndrome diagnosis in the emergency department, new guidelines regarding decisional algorithms have been published by the European Society of Cardiology (ESC) in 2020. Based mostly on ultrasensitive troponin laboratory assay, it is necessary to check their applicability in an emergency laboratory according to the analytical performance of the different devices used.

\section{METHODS}

The laboratory of the University Hospital of Liège has 5 Alinity (Abbott) performing the ultrasensitive troponin I (HsTnI) assay. The ESC guidelines recommend, depending on whether the value of HsTnI at admission is $<5 \mathrm{ng} / \mathrm{L}$ or between 5 and $64 \mathrm{ng} / \mathrm{L}$, a delta of 2 and 6 respectively for the management of the patient care. The potentiel use of a different device for the determination of the second sample for the same patient ( $\mathrm{T}+1$ hour) implies the notion of interanalyzer variability. It is therefore necessary to ensure that the impact of the analytical variability on the result obtained does not exceed the announced delta. Thus, it is needed to ensure the suitability of the algorithm regarding the inter-analytical CV. For the comparison, we analysed successively 24 samples (dry tube) on the 5 Alinity in order to calculate the inter-analyzer analytical CV (CVin).

\section{RESULTS}

Considering the delta announced by the ESC as the biologically significant difference and with the intra-individual biologic variability of HsTnI described in the literature, the theoretical maximum analytical CV (CVa) that should not be exceeded was calculated using the reference change value formula. The CVin of 7,8\% was obtained and compared with the different theoretical maximum CVa depending on the baseline value of HsTnI. Starting from of HsTnI concentration of $27 \mathrm{ng} / \mathrm{L}$ at TO, we demonstrated that the maximum CVa is exceeded by the CVin. 


\title{
CONCLUSION
}

The applicability of the ESC guidelines, in the light of the analytical conditions of the emergency laboratory, is challenging. In our laboratory, the observed CVin allows only a partial use of the algorithm. A solution would be to adapt the delta of the algorithm proposed by the ESC according to each emergency laboratory.

\section{C16}

\section{SUSPICION OF ASPECIFIC TROPONIN I ELEVATION: A CASE REPORT OF TWO INTENSIVE CARE PATIENTS}

\author{
G. Sqalli $^{1}$, V. Huberty ${ }^{2}$, J. De Marchin ${ }^{3}$, E. Cavalier ${ }^{1}$, C. Le Goff ${ }^{1}$ \\ ${ }^{1}$ Departement of Clinical Chemistry, University of Liège, CHU Sart-Tilman, Liège, Belgium, Liege, Belgium, ${ }^{2}$ Department \\ of Laboratory Medicine, CHR of Verviers, Verviers, Belgium, Verviers, Belgium, ${ }^{3}$ Department of Laboratory Medicine, CHR \\ Citadelle, Liège, Belgium, Liège, Belgium
}

\section{BACKGROUND-AIM}

Although the ESC has recommended the use of ultrasensitive Troponin (Tn) I or T, the assay is curently debated because of potentiel lack of specificity. Our work illustrates the biological exploration of two patients from the intensive care unit of the University Hospital of Liege (Belgium) whose Tn results were concerning.

\section{METHODS}

In 2021, a 70-year-old man was admitted to the emergency department (ED) for dyspnea. He later underwent surgery for thromboendarterectomy, triple bypass surgery and aortic valve replacement. Following it, the patient displayed a Tn concentration increase with a maximum of $42,524 \mathrm{ng} / \mathrm{L}$. The second patient was 47 years old and was admitted to the ED for cardiorespiratory arrest. One week later, an acute peripheral ischaemia (API) was diagnosed. TnI results of the patient increased up to $12.639 \mathrm{ng} / \mathrm{L}$ following the embolectomy procedure. In our laboratory, the Alinity Abbott ${ }^{\circledR}$ analysers are used for this assay. Atellica from Siemens ${ }^{\circledR}$, Cobas 8000 from Roche ${ }^{\circledR}$ and Vitros from Ortho clinical Diagnostic $®$ were used for external analysis.

\section{RESULTS}

A significant Tn rise is expected after cardiac surgery and depends on the type of surgery performed. As the value was considered inadequate by the intensivists, it was explored. The result was confirmed by others methods, except the Vitros analyser which showed lower values $(22,300 \mathrm{ng} / \mathrm{L})$. In order to determine if this elevation should be considered abnormal, all the causes of Tn elevations observed in our hospital have been checked during a month $(n=66)$. The median of postoperative value was about 20,000ng/L. Then, a test for anti-heterophilic antibodies was performed and found negative. The elevated Tn T value $(2,391 \mathrm{ng} / \mathrm{L})$ is an additional argument in favour of a mycoardial injury. In conclusion, the exploration leads us to believe that this Tn increase was the consequence of the postoperative period.

As with the first patient, the biological investigation confirmed the initial result for the second one. The hypothesis is that the API which is responsible of rhabdomyolysis also caused myocardial injury on a heart already weakened.

\section{CONCLUSION}

There are still some pitfalls in the interpretation of ultrasensitive Tn. In our cases, the Tn elevation was specific to the heart muscle but the cause was either surgery or rhabdomyolysis. 


\title{
C17
}

\section{COMPARISON OF S-MONOVETTE ® LITHIUM-HEPARIN GEL AND S-MONOVETTE ® LITHIUM-HEPARIN GEL+ ON ALINITY ABBOTT @}

\author{
G. Sqalli $^{1}$, R. Gadisseur ${ }^{1}$, B. Onkelinx ${ }^{1}$, E. Cavalier ${ }^{1}$ \\ ${ }^{1}$ Departement of Clinical Chemistry, University of Liège, CHU Sart-Tilman, Liège, Belgium, Liege, Belgium
}

\section{BACKGROUND-AIM}

Centrifugation time is a limiting step in reducing the Turn-Around-Time (TAT). However, reducing TAT is of capital importance in the clinical course, especially in emergency department (ED). Thus development of methods able to reduce centrifugation time is a great deal of interest. The implementation of the new tubes S-Monovette ${ }^{\circledR}$ Lithium-Heparin Gel+ ( $\mathrm{LH} \mathrm{Gel+)} \mathrm{could} \mathrm{be} \mathrm{a} \mathrm{solution} \mathrm{as} \mathrm{these} \mathrm{require} \mathrm{half} \mathrm{the} \mathrm{centrifugation} \mathrm{time} \mathrm{than} \mathrm{previous} \mathrm{LH} \mathrm{Gel.} \mathrm{The} \mathrm{aim} \mathrm{of} \mathrm{the} \mathrm{study} \mathrm{is} \mathrm{to}$ prove the clinical equivalence between both of these tubes. Therefore, we compared 64 frequently requested clinicalchemical and immunological parameters in plasma by Alinity Abbott ${ }^{\circledR}$ analyzers and the commutability of samples.

\section{METHODS}

To evaluate the commutability of samples, we selected 42 patients coming from different departments: 7 patients from ED, intensive care unit (ICU), dialysis, oncology, 7 pregnant women and we included 7 healthy individuals (HI). For each individual, 4 blood sample tubes were drawn: 2 tubes LH Gel+ and 2 tubes LH Gel. All the samples were immediately transported to the laboratory. The LH Gel tubes were centrifuged in the "Centrifuge 5810" (Eppendorf) for 10 minutes at 3000g. At the same moment, the LH Gel+ tubes were centrifuged in the "Dash Apex Series" (Drucker Diagnostics) for 5 minutes at 3000g. Then, we measured 64 parameters on each plasma matrix, the same day, in the same run. The measurements were plotted against each other and Passing-Bablok regressions was calculated.

\section{RESULTS}

In all patient groups and among all parameters, 43 parameters did not show any statistically significant difference (Confidence Interval 95\% (CI95) of the slope included 1 and CI95 of intercept included 0). For the parameters which results displayed proportional and/or systematic errors, the mean difference was calculated and compared to the acceptable change limit (ACL) and the total change limit (TCL) as described by EFLM. CK and insulin results exceeded the ACL, however none of the biases exceeded the TCL.

\section{CONCLUSION}

The LH Gel+ with a short centrifugation time of 5 minutes is suitable for a medical use as it showed clinical equivalence with the LH Gel; especially for the ED. The LH Gel and LH Gel+ are commutable.

\section{C18}

\section{VERIFICATION OF THE FULLY AUTOMATED LC-MS/MS CASCADION INSTRUMENT FOR THE ANALYSIS OF BLOOD IMMUNOSUPPRESSANTS}

\author{
E. Mathieu $^{1}$, D. Fage ${ }^{1}$, C. Duterme ${ }^{1}$, F. Cotton ${ }^{1}$ \\ ${ }^{1}$ LHUB-ULB, Brussels, Belgium

\section{Objectives}

The goal of this study was to perform the verification of the Cascadion SM Immunosuppressants Panel (tacrolimus, cyclosporin, everolimus and sirolimus) and to assess the general workflow on this first fully automated LC-MS/MS instrument. 


\title{
Material and methods
}

After assessment of the precision (repeatability and intermediate fidelity on both channels) and the linearity (data not shown), a comparison was done on clinical samples ( $\mathrm{n}=30$ - 49 depending on the immunosuppressant drug (ISD)) between 2 Cascadion SM Clinical Analyzers (ThermoFisher, Vantaa, Finland) and the MassTox ISD assay (Chromsystems, Munich, Germany) run on a 6460 LC-MS/MS Agilent system. External quality controls (EQC) from LGC were analyzed on one Cascadion to assess the accuracy.

The acceptability criteria used were issued from the IATDMCT ( $\mathrm{CV}<6 \%$, bias between 2 LC-MS/MS methods $\leq 10 \%$ ). Additional experiments were performed to challenge the limits of the instrument (e.g. carry-over, impact of haematocrit or sedimentation) and to assess its integration into a core lab. The turnaround-time (TAT) impact of this automated method was estimated after a routine day.

\section{Results}

The bias between the 2 Cascadion was $<3.2 \%$ for the 4 ISD. The comparison with the MassTox ISD assay showed an unacceptable bias (-11.6\%) for cyclosporin only. However, the external quality controls results on Cascadion presented a very low bias $(0.8 \%, n=10)$, suggesting a better accuracy of this method.

Additional experiments showed good performances. The random accessibility and the ease of use by nonspecialized allowed a wider working time range and a reduction of TAT of about $65 \%$.

\section{Conclusion}

Our study showed that the Cascadion SM Immunosuppressants Panel held its promises in term of analytical performances, workflow aspects and ease of use by non-specialized lab technicians.

\section{C19}

\section{VALUATION OF UPDATED THYROID ELECSYS® IMMUNOASSAYS WITH DECREASED SUSCEPTIBILITY FOR BIOTIN INTERFERENE}

\author{
$\underline{\text { A. Evenepoel }}^{1}$, J. Schiettecatte ${ }^{1}$, A. van Dalem ${ }^{1}$, E. Anckaert ${ }^{1}$
}

${ }^{1}$ Vrije Universiteit Brussel (VUB), Universitair Ziekenhuis Brussel (UZ Brussel), Departement of Clinical Biology, Laarbeeklaan 101, 1090 Brussel, Belgium

\section{Objective}

To evaluate four newly updated Elecsys ${ }^{\circledR}$ immunoassays for thyroid stimulating hormone (TSH), TSH receptor antibodies (TRAb), thyroglobulin (Tg) and free triiodothyronine (FT3).

\section{Materials and methods}

The evaluation of four Elecsys ${ }^{\circledR}$ ECLIA immunoassays (TRAb, TSH, Tg and FT3) was executed on the Cobas 8000 e801 system (Roche Diagnostics, Switzerland) by verification of the precision (CV\%; low \& high), accuracy (bias\%; EQAS Immunoassay), susceptibility to biotin interference and the comparison between old and new assay results.

\section{Results}

For each assay, we found that both bias and imprecision were acceptable when compared to the Ricos criteria (table 1). Non-significant differences between the biotin sensitive and resistant version were observed using the Ricos criteria for acceptable bias between methods (data not-shown). Biotin spiking experiments revealed that the manufacturers' claims on the new biotin sensitivity threshold were valid (table 1). 
Table 1: Analytical performance of the updated thyroid markers

\begin{tabular}{|c|c|c|c|c|c|c|c|c|c|}
\hline & $\begin{array}{l}\text { Wit } \\
\text { day }\end{array}$ & $\begin{array}{l}\text { hin- } \\
\text { CV\% }\end{array}$ & Tota & CV\% & $\begin{array}{r}\text { CV\% } \\
\text { criterion }\end{array}$ & $\begin{array}{r}\text { Assay bias\% } \\
\text { (EQC) }\end{array}$ & $\begin{array}{r}\text { Bias\% } \\
\text { criterion }\end{array}$ & $\begin{array}{r}\text { Experimentally validated biotin } \\
\text { threshold }(\mathrm{ng} / \mathrm{mL})\end{array}$ & $\begin{array}{r}\text { Insert biotin threshold } \\
(\mathrm{ng} / \mathrm{mL})\end{array}$ \\
\hline Level & Low & High & Low & High & & & & & \\
\hline TRAb & 3.97 & 1.42 & 4.22 & 1.44 & 2.40 & I & I & 1000 & 600 \\
\hline TSH & 1.04 & 1.20 & 2.01 & 1.41 & 9.70 & -2.17 & 7.80 & 1300 & 1200 \\
\hline $\mathrm{Tg}$ & 1.02 & 0.92 & 1.97 & 2.33 & 7.00 & -7.48 & 10.40 & 1560 & 1200 \\
\hline FT3 & 1.50 & 1.90 & 2.00 & 1.90 & 4.00 & -1.61 & 4.80 & 1560 & 1200 \\
\hline
\end{tabular}

\title{
Conclusion
}

Biotin is frequently used as a beauty supplement or as treatment for metabolic diseases in high doses. Exogenous biotin may interfere in immunoassays, causing falsely decreased (sandwich immunoassays: Tg, TSH) or falsely increased (competitive immunoassay: TRAb, FT3) results. Method comparison showed that new versions generate similar results as the previous assays (not shown). The updated Elecsys ${ }^{\circledR}$ assays showed excellent analytical performance with a superior resistance to biotin. The newly updated assays allow accurate and reliable measurements, which could avoid misdiagnosis and improve follow-up in patients with thyroid diseases, even when biotin is taken.

\section{C20}

\section{ASPARAGINASE-INDUCED PSEUDOHYPONATREMIA, A CASE-DRIVEN WORKING STRATEGY IN PEDIATRIC HEMATO-ONCOLOGIC PATIENTS}

\author{
$\underline{\text { A. Evenepoel }}^{1}$, P. Herroelen ${ }^{1}$, K. Lanckmans ${ }^{1}$, J. van der Werff Ten Bosch ${ }^{2}$, M. Martin ${ }^{1}$, I. Weets ${ }^{1}$, A. van Dalem ${ }^{1}$ \\ ${ }^{1}$ Vrije Universiteit Brussel (VUB), Universitair Ziekenhuis Brussel (UZ Brussel), Departement of Clinical Biology, Laar- \\ beeklaan 101, 1090 Brussel, Belgium, ${ }^{2}$ Vrije Universiteit Brussel (VUB), Universitair Ziekenhuis Brussel (UZ Brussel), \\ Departement of Pediatric Hemato-oncology, Laarbeeklaan 101, 1090 Brussels, Belgium
}

\section{Objective}

Validating a patient friendly working strategy for reliable sodium determination in high lipemic samples

\section{Materials and methods}

Sodium and total protein (TP) were analyzed on the Cobas 8000 (Roche Diagnostics, Switzerland) in venous plasma. Sodium in arterial or venous whole blood was determined on the ABL90 Flex (Radiometer, Denmark). We evaluated 4 strategies in a pediatric patient with asparaginase-induced pseudohyponatremia, verified the impact of TP on sodium $(n=2274)$ and tested the interchangeability $(n=40)$ of methods (direct vs indirect ISE) and sample types (arterial, venous, mixed up routine venous blood).

\section{Results}

Sodium measured with the indirect ISE, before and after ultracentrifugation, differed significantly from the direct ISE and calculated sodium. With $1500 \mathrm{mg} / \mathrm{dL}$ as triglyceride threshold, significant difference between direct and indirect ISE was found. TP influenced Na when TP $>83 \mathrm{mg} / \mathrm{mL}$, with $22 \%$ of biases exceeding the criterion (EKE $\mathrm{d} \%=3.4 \%$ ), which is 7-times higher than the group of TP $65-83 \mathrm{mg} / \mathrm{L}$. The interchangeability study did not reveal any significant differences between the three groups of sample types and analysers. Passing-Bablok curves and Bland-Altman test showed comparability. 


\title{
Conclusion
}

Indirect ISE suffered from high triglycerides, without lipemic indexes exceeding insert cutoffs. Direct ISE was insensitive to hypertriglyceridemia. Direct ISE results on whole blood, after mixing up, and direct ISE results on blood gas tubes were statistically comparable. We propose the consecutive use of only one Li-heparin venous sample for both analyzers. Samples can follow the regular workflow and a reflex-analysis will be performed on the ABL Flex on the same tube, preventing re-sampling in these patients. Resulting in less patient burden and stress which is beneficial for patient care. In future cases of hypertriglyceridemia, a triglyceride threshold of $1500 \mathrm{mg} / \mathrm{dL}$ will be used for reliable sodium determination, until confirmation of a more specific L-index threshold for sodium.

\section{C21}

\section{HEMOLYSIS INDEX OPTIMISATION FOR REPORTING AND INTERPRETING LABORATORY RESULTS}

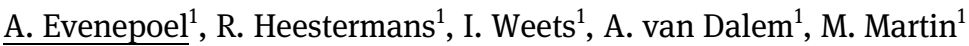 \\ ${ }^{1}$ Vrije Universiteit Brussel (VUB), Universitair Ziekenhuis Brussel (UZ Brussel), Departement of Clinical Biology, Laar- \\ beeklaan 101, 1090 Brussel, Belgium
}

\section{Objective}

We optimized the H-index interpretation for Cobas 8000 systems (Roche Diagnostics, Switzerland) by comparing acceptability criteria (EFLM, Ricos, GLICK). Creating a practical overview with parameter specific degrees of hemolysis interference.

\section{Material and Methods}

Healthy subjects $(n=20)$ and cardio-pathologic patient samples $(n=10)$ were included and tested on the Cobas 8000 system (ISE, c702, e801). H-index was determined with the SI2 kit (Roche Diagnostics, Switzerland). Hemolysis thresholds of 24 hemolysis sensitive assays were evaluated by the GLICK protocol. The bias for each individual parameter and hemolysis level is compared to the EFLM, Ricos and GLICK (10\%) threshold.

\section{Results}

Almost all tested parameters were in agreement with the trends found in the inserts provided by Roche Diagnostics. For all parameters, the EFLM database was the primary source for acceptance criteria. If absent in the EFLM database, we used the other databases. Ricos criteria were used for five parameters and GLICK for one. This resulted in an interpretation scale, taking into account the degree of hemolysis and specific impact on each parameter. The thresholds for ALP, AST, creatinine, P and LDH were similar to those of the insert. Thresholds for i.e. albumin and bicarbonate were lower than those of the insert, using the EFLM criterion. On the contrary, insert thresholds for assays such as amylase or K were stricter than found, if EFLM criteria were used. Two populations of hs-TnT were evaluated. In healthy subjects, hardly any interference was seen, whereas pathological samples revealed higher significant biases.

\section{Conclusion}

Hemolysis is a major cause of sample interference. We developed a novel in-depth interpretation scale of the H-index to support both the clinician and laboratory about the hemolysis impact with a better grasp at when to resample. In addition, more precise analytical flags and alarms (mild/moderate/severe increase/decrease) can be implemented. Leading to less patient burden and to achieve a more accurate diagnosis. 


\title{
C22
}

\section{THE IMPORTANCE OF PEAK TAILING FLAGS IN HBA1C ANALYSIS FOR THE DETECTION OF UNEXPECTED RARE HEMOGLOBIN VARIANTS IN DIABETIC CAUCASIAN PATIENTS}

\author{
$\underline{\text { A. Evenepoel }}^{1}$, F. Cotton ${ }^{2}$, I. De Waele ${ }^{3}$, B. Gulbis ${ }^{2}$, I. Weets ${ }^{1}$ \\ ${ }^{1}$ Vrije Universiteit Brussel (VUB), Universitair Ziekenhuis Brussel (UZ Brussel), Departement of Clinical Biology, Laar- \\ beeklaan 101, 1090 Brussel, Belgium, ${ }^{2}$ Université Libre de Bruxelles (ULB), Laboratoire Hospitalier Universitaire de \\ Bruxelles (LHUB-ULB), Departement of Clinical Biology, Lenniksebaan 808, 1070 Brussels, Belgium, ${ }^{3}$ Sebia, Jan Olie- \\ slagerslaan 41, 1800 Vilvoorde, Belgium
}

\section{Objective}

Identifying the cause of abnormal HbA1c peaks and tailing with ion-exchange chromatography (HPLC) in a diabetic Caucasian patient.

\section{Material and Methods}

HbA1c was determined on the Bio-rad D100 (Bio-rad, Hercules, USA) and Affinion (Abbott, USA). Fructosamine was measured on the Cobas 8000 (Roche diagnostics, Switzerland). Hemoglobin variant analysis was performed on the Bio-rad Variant II (Bio-rad, Hercules, USA) and Capylaris III (Sebia, Belgium). Chromatographs were compared with Bio-Rad Hemoglobin Library.

\section{Results}

D100 analysis revealed an HbA1c result of 7.3\%, indicating uncontrolled diabetes and need for therapy adjustment. Closer inspection of the D100 chromatogram showed an abnormal HbA1c peak with peak tailing flags. Both Affinion HbA1c results (5.8\%) and fructosamine levels ( $236 \mu \mathrm{mol} / \mathrm{L})$ were normal, in contrast to the elevated D100 HbA1c. Variant II analysis seemed normal at first glance, except for an earlier $\mathrm{HbA}_{0}$ retention time (2.26 min) and blunt $\mathrm{P} 2$ and $\mathrm{P} 3$ peaks. Capillary electrophoresis (CE) confirmed the presence of a hemoglobin variant. Phenotypically we concluded that the patient is heterozygous for $\mathrm{Hb}$ Athens-Georgia. Genotyping has been requested to identify the variant, but the result is not yet available.

\section{Conclusion}

Hemoglobinopathies are uncommon in northern European Caucasian populations. Recent case reports however, describe the presence of hemoglobin variants in this population. These undiagnosed variants could interfere with HbA1c analysis. Peeters et al saw that HbA1c analysis with CE does not suffer from the presence of $\mathrm{Hb}$ Athens-Georgia. We observed the opposite with HPLC determination, where falsely elevated results were found. Presence of peak tailing flags could be an indication of hemoglobin variants and chromatograms should be closely inspected. Hemoglobin variants detected with HbA1c methods should be mentioned in order to offer the possibility of its identification. Nevertheless, analytical issues should always be kept in mind. HbA1c borate affinity chromatography (Affinion), which is not influenced by these rare variants, and fructosamine are good alternatives for the follow-up of these diabetic patients.

\section{C23}

\section{CONCORDANCE OF APOLIPOPROTEIN B CONCENTRATION WITH THE FRIEDEWALD, MARTIN-HOPKINS, AND SAMPSON EQUATIONS FOR CALCULATING LDL CHOLESTEROL}

\author{
$\underline{\text { P. L. Briers }}{ }^{1}$, M. R. Langlois ${ }^{2}$ \\ ${ }^{1}$ Department of Laboratory Medicine, AZ St-Jan Hospital, Brugge and Catholic University Leuven, Belgium., Brugge, \\ Belgium, ${ }^{2}$ Department of Laboratory Medicine, AZ St-Jan Hospital, Brugge, Belgium. Department of Diagnostic Sciences, \\ Faculty of Medicine and Health Sciences, Ghent University, Ghent, Belgium., Brugge, Belgium
}




\section{Objective}

Two new equations, the Martin-Hopkins and the Sampson equation, were recently developed to overcome shortcomings of the Friedewald equation for calculating LDL-cholesterol that have become more relevant with the introduction of new lipid-lowering therapies and lower LDL-cholesterol therapeutic goals. We aimed to compare the concordance of the two equations with apolipoprotein $\mathrm{B}(\mathrm{apoB})$, a surrogate marker of the number of LDL particles.

\section{Materials and methods}

In a study of serum lipid data of 1179 patients who consulted the AZ St.-Jan Hospital Bruges, the correlation and concordance of the Friedewald, Martin-Hopkins and Sampson equations with apoB concentration, measured by immunonephelometry, were determined and compared.

\section{Results}

The Martin-Hopkins equation showed significantly higher correlation than the Friedewald equation with apoB in the entire dataset and in the subgroup of patients with low LDL-cholesterol $<70 \mathrm{mg} / \mathrm{dL}$. Both Martin-Hopkins and Sampson equations improved the concordance of LDL-cholesterol with regard to treatment group classification based on population-equivalent thresholds of apoB, with the best concordance (75.6\%) obtained using Martin-Hopkins equation (vs. $70.2 \%$ with Sampson and 60.5\% with Friedewald) in hypertriglyceridemic patients (175-400 mg/dL).

\section{Conclusion}

LDL-cholesterol calculated by the Martin-Hopkins (and, to a lesser extent, Sampson) equation is more closely associated with the number of LDL particles than Friedewald-calculated LDL-cholesterol. This, in combination with current evidence surrounding the lesser accuracy of the Friedewald equation, is an argument to switch from Friedewald to a modified, improved equation.

\section{C24}

\section{ANALYTICAL PERFORMANCE OF NOVEL SERUM FREE LIGHT CHAIN ASSAYS}

$\underline{\text { M. Cornette }}^{1}$, M. Vercammen ${ }^{2}$, L. Nevejan ${ }^{3}$, P. J. Briers ${ }^{2}$, D. Deeren ${ }^{4}$, J. van Droogenbroeck ${ }^{5}$, K. Fostier ${ }^{6}$, D. De Smet ${ }^{1}$, L. van Hoovels $^{3}$

${ }^{1}$ Department of Laboratory Medicine, AZ Delta Hospital, Roeselare, Roeselare, Belgium, ${ }^{2}$ Department of Laboratory Medicine, Sint-Jan Hospital, Brugge/Oostende, Brugge, Oostende, Belgium, ${ }^{3}$ Department of Laboratory Medicine, OLV Hospital, Aalst, Aalst, Belgium, ${ }^{4}$ Department of Hematology, AZ Delta Hospital, Roeselare, Roeselare, Belgium, ${ }^{5}$ Department of Hematology, Sint-Jan Hospital, Brugge/Oostende, Brugge, Oostende, Belgium, ${ }^{6}$ Department of Hematology, OLV Hospital, Aalst, Aalst, Belgium

\section{Objectives}

Serum free light chain (sFLC) measurements are part of diagnostic workup of monoclonal gammopathies, but suffer from inherent analytical limitations. We evaluated analytical performance of two new sFLC assays (Diazyme Roche and Sebia ELISA) in comparison with Freelite (The Binding Site, TBS) methods.

\section{Material and Methods}

Assessment of linearity (CLSI EP6-A1) and imprecision (CLSI EP5-A3) was done for 5 polyclonal sFLC assay/platform combinations: Freelite/Optilite method (TBS), Freelite/Cobas c501 (TBS/Roche), Diazyme/Cobas c501 (Roche), Diazyme/ Cobas c503 (Roche) and Sebia FLC $\kappa$ and $\lambda$ ELISA (on AP22 ELITE, Das Italy). Performance characteristics were compared with different quality goals derived from biological variation, manufacturer's claims, and peer group EQA. Method comparison (CLSI-EP9-A3) was done for 120 patient samples, considering Freelite/Optilite as reference method. 


\section{Results}

Total imprecision coefficients $\left(\mathrm{CV}_{\mathrm{t}}\right)$ were comparable, except for Sebia ELISA showing much higher $\mathrm{CV}_{\mathrm{t}}$ 's. Stringent criteria based on biological variation were not fulfilled for most assays. Sebia $\lambda$ ELISA violated all proposed quality goals. Linearity was acceptable for most assays, with correlation coefficients $\left(\mathrm{R}^{2}\right)$ for monoclonal and polyclonal sFLC dilutions ranging from 0.823 to 0.999 for $\kappa$ and from 0.890 to 0.999 for $\lambda$. Lowest linearity $\left(\mathrm{R}^{2}\right)$ results were obtained for $\kappa$ Diazyme/ Cobas c501 and $\lambda$ Sebia ELISA. Method comparison showed excellent correlation $\left(\mathrm{r}_{\mathrm{s}}\right)$ with Freelite/Optilite method for all assays. However, large proportional negative bias was shown for both Sebia $\kappa$ and $\lambda$ ELISA. Also, significant positive bias in the low range $(<10 \mathrm{mg} / \mathrm{L})$ was shown for Freelite/Cobas c501 method, confirming known overrecovery of $\lambda$ sFLC.

\section{Conclusions}

Analytical performance was acceptable. However, (large) differences in nominal sFLC concentrations between assays and platforms hamper interchangeability of sFLC results for diagnostic workup and disease monitoring.

\section{C25}

\section{MIR-143/MIR-145 RELATIVE EXPRESSION PROFILE IS ASSOCIATED WITH ADIPOMYOKINES DYSREGULATION IN THE PATHOLOGICAL ADIPOSITY AND DYSLIPIDEMIC SETTING IN AGING}

R. E. Navarro-Hernandez ${ }^{1}$ J. A. Robles-De Anda ${ }^{2}$, F. I. Corona-Meraz ${ }^{3}$, P. M. Madrigal-Ruiz ${ }^{1}$, J. Castro-Albarran ${ }^{4}$, J. A. Noboa-Velastegui ${ }^{5}$, A. L. Fletes-Rayas ${ }^{6}$

${ }^{1}$ Departamento de Biología Molecular y Genómica. UDG-CA-701, Grupo de Investigación Inmunometabolismo en Enfermedades Emergentes, Centro Universitario de Ciencias de la Salud, Universidad de Guadalajara, GUADALAJARA, Mexico, ${ }^{2}$ Doctorado en Ciencias en Biología Molecular en Medicina. Secretaria Académica. UDG-CA-701, Grupo de Investigación Inmunometabolismo en Enfermedades Emergentes. Centro Universitario de Ciencias de la Salud, Universidad de Guadalajara, GUADALAJARA, Mexico, ${ }^{3}$ Departamento de Ciencias Biomédicas, División de Ciencias de la Salud, Centro Universitario de Tonalá, Universidad de Guadalajara, GUADALAJARA, Mexico, ${ }^{4}$ Departamento de Ciencias de la Salud y Ecología Humana, División de Desarrollo Regional, Centro Universitario de la Costa Sur, AUTLAN, Mexico, ${ }^{5}$ Doctorado en Ciencias Biomédicas. Secretaria Académica. UDG-CA-701, Grupo de Investigación Inmunometabolismo en Enfermedades Emergentes. Centro Universitario de Ciencias de la Salud, Universidad de Guadalajara, GUADALAJARA, Mexico, ${ }^{6}$ Instituto de Investigación en Enfermería y Salud Traslacional. Departamento de Enfermería Aplicada. Centro Universitario de Ciencias de la Salud, Universidad de Guadalajara, GUADALAJARA, Mexico

\section{Objective}

Irisin and adiponectin are adipomyokines that possess crucial roles in metabolism. Also, the presence of microRNAs was involved in metabolic control. Remarkably, circulating miR-143 and miR-145 have shown controversial results regarding women-metabolism in the aging process. Our study aimed to evaluate the association of adipomyokines levels and metabolic profile with the relative expression of circulating miR-143 and miR-145 in the pathological adiposity in the elderly women group.

\section{Material and methods}

We included 60 women, classified as young and senior (aged 20-39 and 40-59 years) by \% of body fat storage. We evaluated body fat storage using bioelectrical impedance. Biochemical markers by routine methods. Insulin, irisin, and adiponectin-oligomers serum levels by the ELISA method. Relative expression measures of $m i R-143$ and $m i R-145$ circulating levels (normalized with endogenous miR-320a) with the TaqMan Advanced miRNA Assays system ${ }^{\circledR}$ and $2^{-\Delta \mathrm{C}}$ method, were assessed in association with clinical outputs. 


\section{Results}

We observed an increase in lipid profile, whereas total and adiponectin oligomers and irisin-adiponectin related indexes were lower in young and seniors' overweight (fat mass $>35 \%$ ) women, parallel to lower miR-145 relative expression levels [0.6-fold change]. As well, the following rho correlations were observed $(P<0.05)$ : miR-143 relative expression levels correlate with miR-145 relative expression [0.431], MMW+LMW-Adiponectin [0.318], insulin [- 0.402] and HOMA-B [0.354]. miR-145 correlate with LDLc profile [0.284 to 0.366] and, \%sdLDL [- 428].

\section{Conclusion}

miR-145 relative expression and adipomyokines lower levels in overweight and, the association with miR-143 relative expression levels suggesting a response to chronic metabolic dysregulation originating in the pathological corporal redistribution of fat mass and dyslipidemic phenotype that is shown in aging.

\section{C26}

\section{FREE LIGHT CHAIN ANTIGEN EXCESS DETECTION IN THREE POLYCLONAL ASSAYS ON FOUR ANALYTICAL PLATFORMS}

$\underline{\text { P. L. Briers }}{ }^{1}$, L. Nevejan ${ }^{2}$, M. Cornette ${ }^{3}$, L. van Hoovels ${ }^{2}$, D. De Smet ${ }^{3}$, D. Deeren ${ }^{4}$, J. van Droogenbroeck ${ }^{5}$, K. Fostier ${ }^{6}$, M. Vercammen ${ }^{7}$

${ }^{1}$ Department of Laboratory Medicine, Sint-Jan Hospital, Brugge, Belgium, ${ }^{2}$ Department of Laboratory Medicine, OLV Hospital, Aalst, Belgium, ${ }^{3}$ Department of Laboratory Medicine, AZ Delta Hospital, Roeselare, Belgium, ${ }^{4}$ Department of Hematology, AZ Delta Hospital, Roeselare, Belgium, ${ }^{5}$ Department of Hematology, Sint-Jan Hospital, Brugge, Belgium, ${ }^{6}$ Department of Hematology, OLV Hospital, Aalst, Belgium, ${ }^{7}$ Research group REIM Vrije Universiteit Brussel (VUB). Department of Laboratory Medicine, Sint-Jan Hospital, Brugge, Belgium

\section{Objective}

To evaluate antigen excess (AGE) detection of three polyclonal serum free light chain (sFLC) assays on four analytical platforms.

\section{Materials and methods}

sFLC concentration was measured using Diazyme sFLC (Roche) on Cobas c501 and c503 (Roche), Sebia sFLC ELISA (Sebia) on AP22 ELITE (DAS) and Freelite (The Binding Site) on Cobas c501, vs Freelite on Optilite (The Binding Site) as a reference. Twenty-eight kappa and 28 lambda samples with suspected AGE were selected based on kinetic flagging with Freelite ("high activity") on Optilite or on Cobas ("kin"). AGE on Optilite was defined as a four-fold higher result of the forced higher dilution vs the lower automatic dilution without flagging ${ }^{1}$. For all other methods, the automatic results were compared with the extrapolated ones, obtained using the assay-specific equation from the method comparison. Again, AGE was considered in case of a four-fold difference. For Diazyme reagents, AGE was calculated in both ways.

\section{Results}

Freelite on Optilite or on Cobas didn't present AGE for kappa nor lamba sFLC. Regarding kappa, Diazyme missed 3/28 samples, two on c501, two on c503 and one on both systems; Sebia didn't miss AGE. For lambda, 3/28 samples presented AGE with Diazyme, both on c501 and c503. Sebia failed to detect AGE in seven samples, two of them also missed by Diazyme. Calculation of AGE by extrapolation generated similar results as by dividing the result of the higher with the automatic dilution (Diazyme), but differed in two samples: one kappa sample was only detected with extrapolation and one lambda sample only with forced dilution.

\section{Conclusion}

We propose a new approach for the calculation of AGE by method comparison. AGE detection with Diazyme and Sebia reagents is suboptimal compared to Freelite on Optilite or on c501. This can lead to postponed or misdiagnosed patients. 


\title{
Reference
}

(1) Vercammen et al 2011 CCA 412:1798

\section{C27}

\section{INTERFERENCE TESTING ON THREE POINT-OF-CARE BLOOD GAS ANALYZERS}

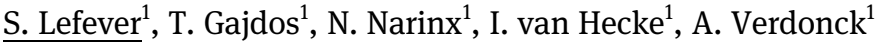 \\ ${ }^{1}$ UZ Leuven, Leuven, Belgium
}

\section{Introduction}

The purpose of this study was to evaluate substances that could influence co-oximetry (CoOX) and electrolyte results on point-of-care blood gas analyzers (BGA). Interference by hemolysis (HEM), lipemia (LIP), benzalkonium chloride (BzCl) and methylene blue (MB) was investigated.

\section{Materials \& methods}

Three BGA were evaluated: (1) GEM® Premier ${ }^{\mathrm{TM}} 5000$ (GP5000, Werfen, Barcelona, Spain), (2) RAPIDPoint ${ }^{\circledR} 500 \mathrm{e}$ (RP500e, Siemens Healthineers, Erlangen, Germany), and (3) ABL90 Flex Plus (Radiometer Medical ApS, Brønshøj, Denmark). Heparinized whole-blood samples from healthy volunteers were collected by venous puncture, after which they were spiked with the interfering substance of interest. HEM interference was studied by spiking a freeze-thawed whole-blood sample in fresh whole-blood samples to reach different HEM degrees. LIP interference was investigated by spiking SmofLipid® (Fresenius Kabi, Bad Homburg, Germany) in three concentrations (0.5, 1.0 and 2.0\%). BzCl interference was tested in three different concentrations (5, 10 and $20 \mathrm{mg} / \mathrm{L})$ and $\mathrm{MB}$ in four $(5,10,40$ and $80 \mathrm{mg} / \mathrm{L})$. Samples were offered in triplicate to each BGA for every concentration of the interfering substance, together with a blank whole-blood sample.

\section{Results}

All BGA experienced equal positive interference of HEM on potassium determination (up to $+54 \%$ ). BzCl positively interfered with the sodium measurement on ABL90 (up to +11\%) and RP500e (no result), but not on the GP5000. Small interferences due to LIP were observed on CoOX results on all BGA, while only hematocrite on GP5000 was positively influenced by LIP (up to $+17 \%$ ). MB influenced CoOX results equally on all three BGA and sodium results on ABL90 (up to $+13 \%$ ) and RP500e (up to $+9 \%$ ).

\section{Conclusion}

All BGA experienced interference by the tested substances to a greater or lesser extent. Each laboratory should know for their BGA which interfering substances are important and to which extent, and should inform their clinicians about this.

\section{C28}

\section{ANALYTICAL PERFORMANCE OF THREE POINT-OF-CARE BLOOD GAS ANALYZERS}

\author{
$\underline{\text { N. Narinx }}{ }^{1}$, T. Gajdos ${ }^{1}$, S. Lefever ${ }^{1}$, I. van Hecke ${ }^{1}$, A. Verdonck ${ }^{1}$ \\ ${ }^{1}$ Univserity Hospitals Leuven (UZ Leuven), Leuven, Belgium

\section{Objective}

This study was designed to evaluate the analytical performance of three point-of-care blood gas analyzers (BGA) for determination of $\mathrm{pH}$, partial carbon dioxide pressure $\left(\mathrm{pCO}_{2}\right)$, partial oxygen pressure $\left(\mathrm{pO}_{2}\right)$, total hemoglobin $(\mathrm{tHb})$, hematocrit (Hct), sodium $\left(\mathrm{Na}^{+}\right)$, potassium $\left(\mathrm{K}^{+}\right)$, chloride $\left(\mathrm{Cl}^{-}\right)$, calcium $\left(\mathrm{Ca}^{2+}\right)$, lactate (Lac) and glucose $(\mathrm{Glu})$. 


\title{
Materials and methods
}

Three BGA were evaluated: (1) GEM® Premier ${ }^{\mathrm{TM}} 5000$ (GP5000, Werfen, Barcelona, Spain), (2) RAPIDPoint® 500e (RP500e, Siemens Healthineers, Erlangen, Germany), and (3) ABL90 Flex Plus (Radiometer Medical ApS, Brønshøj, Denmark). Analytical performance of each system was assessed using three levels [low (L), intermediate (I), high (H)] of their respective quality control $(\mathrm{QC})$ materials. Total imprecision $\left(\% \mathrm{CV}_{\mathrm{t}}\right)$ and estimated total error (TE) were calculated according to the CLSI-EP15-A3 guideline. Results were verified against preset specifications, in following order if available: (1) d-values by Sciensano (Belgian Health Authorities, Elsene, Belgium) and (2) Ricos Desirable Biological Variation Database specifications. For $\mathrm{pO}_{2}$ no criteria were available from both sources.

\section{Results}

Results for $\% \mathrm{CV}_{\mathrm{t}}$ were acceptable, except for some parameters where a minor deviation existed compared to the preset criteria: GP5000 [tHb (L) and Glu (L)] and RP500e $\left[\mathrm{Na}^{+}(\mathrm{H})\right]$. More deviations from specification criteria were observed for TE: GP5000 [tHb (L-I), $\mathrm{Cl}^{-}(\mathrm{L}), \mathrm{Ca}^{2+}$ (L)], RP500e $\left[\mathrm{Na}^{+}\right.$(L-I-H), Cl- (H), Ca ${ }^{2+}$ (L), Lac (L)], and ABL90 $\left[\mathrm{Na}^{+}(\mathrm{L}-\mathrm{I}), \mathrm{Ca}^{2+}(\mathrm{L}-\mathrm{I}-\mathrm{H})\right]$.

\section{Conclusion}

For both $\% \mathrm{CV}_{\mathrm{t}}$ and $\mathrm{TE}$, not all preset criteria were met, but more deviations were observed for TE compared to $\% \mathrm{CV}_{\mathrm{t}}$. Two parameters, $\mathrm{Na}^{+}$and $\mathrm{Ca}^{2+}$, require more attention as they deviated on one or more QC levels, on two BGA (RP500e and ABL90) and on all three BGA respectively. These findings are in line with the last rounds (2019-2021) of external QC evaluation by Sciensano. Further investigation with patient samples could be of interest.

\section{C29}

\section{EVALUATION OF ETHYLENE GLYCOL INTERFERENCE WITH LACTATE MEASUREMENT ON THREE POINT-OF-CARE BLOOD GAS ANALYZERS}

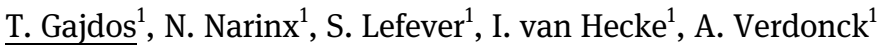 \\ ${ }^{1}$ University Hospitals Leuven (UZ Leuven), Leuven, Belgium

\section{Objective}

Ethylene glycol (EG) and its metabolites [glycolic acid (GA, main metabolite), glyoxylic acid (GXA), oxalic acid (OA), formic acid (FA)] are known to interfere with lactate (Lac) analysis on some point-of-care blood gas analyzers (BGA), but not on laboratory automates. ${ }^{1}$ This discrepancy, in literature described as the 'lactate gap', could be helpful to quickly diagnose EG poisoning. In this study, the level of interference of EG and its metabolites with lactate determination was evaluated on three BGA.

\section{Material and Methods}

Heparinized whole blood samples from healthy individuals were spiked with GA (2.5, 5, 10, 20 and 40 mmol/L), GXA (20 mmol/L), OX (20 mmol/L), FA (20 mmol/L) and EG (0.014 mmol/L). All samples were analyzed first in triplicate on the three BGA [ABL90 Flex Plus (Radiometer Medical ApS, Brønshøj, Denmark), GEM®Premier ${ }^{\mathrm{TM}} 5000$ (GP5000, Werfen, Barcelona, Spain), and RAPIDPoint ${ }^{\circledR}$ 500e (RP500e, Siemens Healthineers, Erlangen, Germany)]. Afterwards they were analyzed on the Cobas ${ }^{\circledR} 8000$ c702 (Roche Diagnostics, Mannheim Germany).

\section{Results}

All analyzers showed falsely elevated Lac results in the presence of GA and GXA (table 1). The ABL90 was influenced more strongly by GA (up to $63.7 \mathrm{mmol} / \mathrm{L}$ ) in comparison with the other two analyzers (figure 1). Lac results on the Cobas ${ }^{\circledR}$ 
experienced positive interference by GA and GXA as well, however to a lesser extent than on the BGA, with the exception of GXA interference on the RP500e [(Cobas ${ }^{\circledR}(10.9 \mathrm{mmol} / \mathrm{L})$ vs. RP500e $\left.(5.7 \mathrm{mmol} / \mathrm{L})\right]$. EG, OX and FA did not show significant interference on Lac measurement.

\section{Conclusion}

All three BGA experienced a positive interference on Lac measurement by GA and GXA, but not by EG, OX and FA. Interestingly, GA and GXA influenced Lac determination on the Cobas ${ }^{\circledR} 8000$ as well, however to a lesser extent than on the BGA. Therefore, the "lactate gap" between the BGA and the laboratory automate will still exist and should warrant clinicians to consider EG poisoning.

\section{References}

1. Poesen K, Degandt S, Boes J, Vanstapel F, Desmet K, Vermeersch P. Interference of ethylene glycol with lactate measurement: a comparison study on new generation cassette-based blood gas analyzers. Clin Chim Acta. 2012 Dec 24;414:18-9. doi: 10.1016/j.cca.2012.07.013. Epub 2012 Jul 20. PMID: 22841681.

Table 1: Influence of ethylene glycol and its metabolites on lactate determination on a laboratory automate (Cobas@8000 c702) and three blood gas analyzers. Results marked in bold are considered aberrant. Abbreviations: ABL90 = ABL90 Flex Plus, GP5000 = GEM ${ }^{\circ}$ Premier $^{\mathrm{TM}} 5000$ and RP500e $=$ RAPIDPoint ${ }^{\circledR} 500 \mathrm{e}$.

\begin{tabular}{|c|c|c|c|c|}
\hline & Cobas $₫ 8000$ & ABL90 & GP5000 & RP500e \\
\hline Blank & 1.16 & 1.16 & 1.23 & 1.86 \\
\hline Ethylene glycol (0.014 mmol/L) & 1.16 & 1.13 & 1.23 & 1.48 \\
\hline Glycolic acid (20 mmol/L) & 8.90 & 43.67 & $>17$ & 18.98 \\
\hline Glyoxylic acid (20 mmol/L) & $\overline{10.91}$ & 20.33 & 12.97 & $\underline{5.70}$ \\
\hline Oxalic acid (20 mmol/L) & 0.97 & 0.83 & 1.23 & $\overline{1.43}$ \\
\hline Formic acid $(20 \mathrm{mmol} / \mathrm{L})$ & 1.07 & 1.00 & 1.37 & 1.28 \\
\hline
\end{tabular}

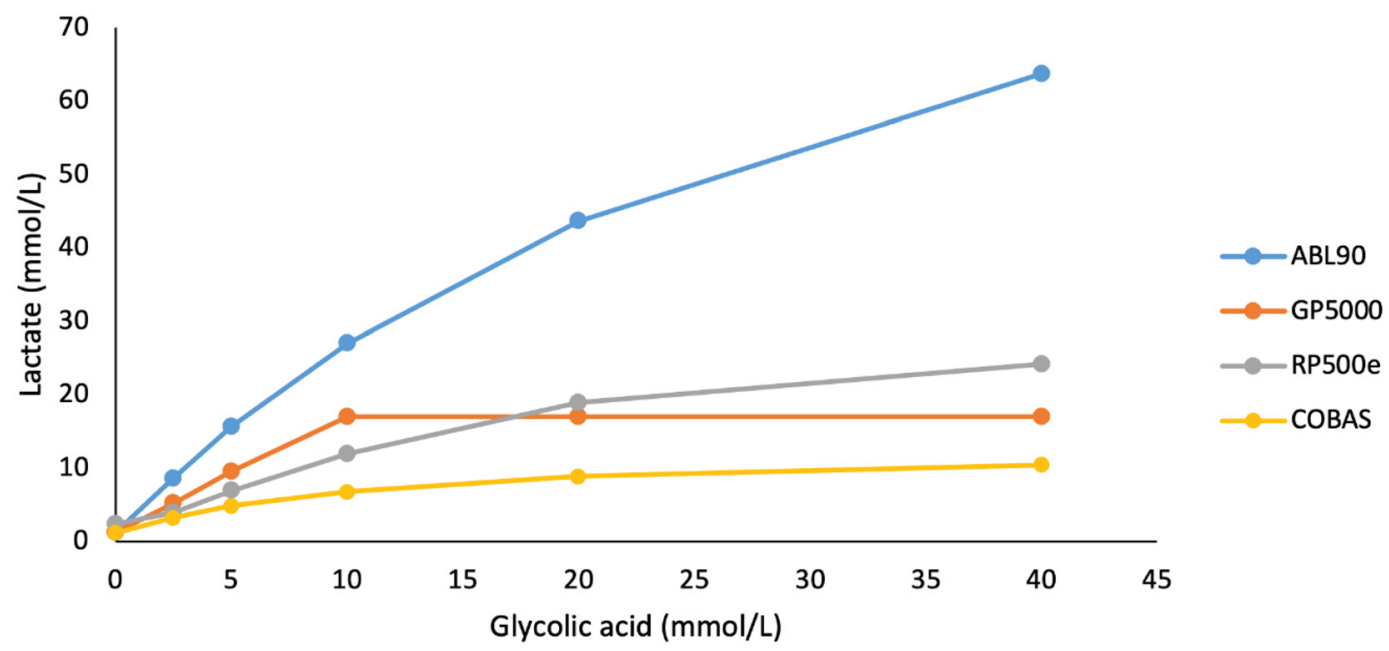

Figure 1: Influence on lactate determination by glycolic acid in increasing concentrations on one routine laboratory automate (Cobas $@ 8000$ ) and three point-of-care blood gas analyzers. Results of the GP5000 deflect starting from a glycolic acid concentration of $10 \mathrm{mmol} / \mathrm{L}$, as the blood gas analyzer reported results as $>17.0 \mathrm{mmol} / \mathrm{L}$ lactate. Abbreviations: $\mathrm{ABL} 90=\mathrm{ABL} 90$ Flex Plus, GP5000 $=\mathrm{GEM} \circledast \mathrm{Premier}^{\mathrm{TM}} 5000$ and $\mathrm{RP} 500 \mathrm{e}=$ RAPIDPoint ${ }^{\circledR} 500$ e. 


\title{
C30
}

\section{VALIDATION OF ANTI-MULLERIAN HORMONE (AMH) ASSAY ON LUMIPULSE G1200 FROM FUJIREBIO}

\author{
A. Ladang ${ }^{1}$, P. Lukas ${ }^{1}$, F. Watar ${ }^{1}$, F. Wolff', E. Cavalier ${ }^{1}$ \\ ${ }^{1}$ Clinical chemistry / CHU de Liège, Liège, Belgium, ${ }^{2}$ Clinical chemistry / LHUB-ULB, Bruxelles, Belgium \\ Validation of assay on automated plateform is an important task in laboratory medicine. Here, we are reporting validation \\ of the LUMIPULSE G AMH assay on the Lumipulse G1200 automated plateform from Fujirebio.
}

To evaluate precision, 4 pools of sera and 2 internal quality controls were tested 5 times a day for 5 days. We found a CV of $10 \%$ for pools at 0.5 and $1.5 \mathrm{ng} / \mathrm{mL}$ and a CV of $1.3,1.1$ and 1.4 for samples at 3.2, 5.1, $12.0 \mathrm{ng} / \mathrm{mL}$, respectively. The pool at a concentration of $0.03 \mathrm{ng} / \mathrm{mL}$ did not reach a CV below $20 \%$. Thus, the limit of quantification was extrapolated from these data at $0.15 \mathrm{ng} / \mathrm{mL}$. Therefore, we could not confirm the LOQ of $0.03 \mathrm{ng} / \mathrm{mL}$ provided by the manufacturer.

To establish the method comparison, we compared our results from 42 samples to these obtained on Cobas e602 (Roche device from LHUB-ULB). We found a proportional bias between both methods with a slope of 0.817 ( $95 \%$ confidence interval: 0.754 ; 0.879 ) and but no systematic bias with an intercept of 0.0711 (95\% confidence interval: -0.1654 ; 0.3077$)$.

To check the reference ranges provided by the manufacturer, we measured 20 sera from women aged between 31 and 35 years old, 20 sera from women aged between 36 and 40 years old. All of these 40 samples felt into the reference ranges provided by the manufacturer.

Taken together, our results show that Lumipulse G AMH assay is a valid method to quantify anti-Mullerian hormone. Yet, there is a significant bias with Roche method and specific reference ranges need to be applied.

\section{C31}

\section{PEG PRECIPITATION AS A POTENTIAL TOOL FOR ELIMINATION OF PARAPROTEIN INTERFERENCE ON THYROID FUNCTION TESTS}

\author{
$\underline{\text { J. Vercruyssen }}{ }^{1}$, M. Berth ${ }^{1}$ \\ ${ }^{1}$ Department of Laboratory Medicine, AZ Alma, Eeklo, Belgium

\section{Objective}

Recently, abnormal thyroid function tests (Cobas, Roche) were found in a patient who had no signs or symptoms of thyroid dysfunction: TSH levels were normal whereas free thyroxine (FT4) and free triiodothyronine (FT3) levels were decreased. Similar results were found on Architect (Abbott) and Atellica (Siemens). The patient was known with a stable serum IgG paraprotein of approximately $30 \mathrm{~g} / \mathrm{L}$. Various methods (blocking tubes, absorption with solid phase) failed to show any interference. However, polyethylene glycol (PEG) precipitation of the serum sample resulted in the normalization of FT4 and FT3, with a recovery of $248 \%$ and $138 \%$ respectively.

This case led us to evaluate the PEG precipitation method as a potential approach to overcome paraprotein interference on thyroid function tests.

\section{Material and methods}

A total of 68 serum samples were evaluated: 17 samples from patients with a paraprotein and 51 samples from random control samples. FT4 and FT3 were analyzed on Cobas 8000 before and after PEG precipitation, and analyte recovery was calculated. 


\title{
Results
}

Reproducibility of the PEG precipitation on FT4 and FT3 values was evaluated on two serum samples with two different FT4 levels (10.3 pmol/l and $30.5 \mathrm{pmol} / \mathrm{l})$ and FT3 levels ( $1.79 \mathrm{pmol} / \mathrm{l}$ and $3.83 \mathrm{pmol} / \mathrm{l})$. Mean recovery was 96 and $99 \%$ for FT4, and $86 \%$ and $101 \%$ for FT3, with CV's of the recovery ranging from $1 \%$ to $3 \%$.

For all 68 samples mean FT4 recovery was $99 \% \pm 6 \%$ and mean FT3 recovery was $81 \% \pm 9 \%$. There was no significant difference in recovery between the 17 paraprotein samples and the 51 control samples. Based on mean $\pm 3 \mathrm{SD}$, recoveries between $80 \%$ and 119\% for FT4 and between 53\% and 109\% for FT3 were observed for the total population.

\section{Conclusion}

PEG precipitation may be a suitable and easy tool to evaluate possible immunoglobulin mediated analytical interference in free thyroid hormone assays.

\section{C32}

\section{HEMOX-ANALYZER: ASSESSEMENT OF THE ANALYTICAL PERFORMANCE}

\author{
$\underline{\text { S. Benyaich }}{ }^{1}$, A.-S. Adam ${ }^{1}$, G. Rondelet ${ }^{1}$, F. Cotton $^{1}$, B. Gulbis ${ }^{1}$
}

${ }^{1}$ LHUB-ULB, Bruxelles, Belgium

\section{Background}

Polycythemia can be primitive (Vaquez disease) or secondary. High oxygen affinity hemoglobins (HOAH) are uncommon, often misdiagnosed etiology of secondary erythrocytosis. The diagnosis is based on a decreased p50, phenotypic methods and genetic studies.

The aim of this study was to assess the analytical performance of the HEMOX-ANALYZER (TCS, Southampton, USA) system, for the detection of hemoglobins with altered affinity for as a complementary technique to standard phenotypic methods or prior to molecular genetic analysis.

\section{Materials and methods}

The HEMOX-ANALYZER is an automatic system for determining the oxyhemoglobin dissociation curve (ODC) and P50 values. The oxygen partial pressure is measured by a Clark electrode while the oxyhemoglobin fraction is evaluated by a dual-wavelength spectrophotometer.

The precision of the method was evaluated by analyzing two levels of quality controls (QC 463 EQUIL®).

Repeatability was determined by performing 5 replicates $(n=10)$. Intermediate fidelity by running controls at different temperatures $\left(4^{\circ} \mathrm{C}\right.$ and $\left.-80^{\circ} \mathrm{C}\right)$ over 6 days $(\mathrm{n}=24)$.

Stability was assessed in a healthy volunteer, an anemic patient $(\mathrm{Hb}=6 \mathrm{~g} / \mathrm{dL})$, and a newborn at $4^{\circ} \mathrm{C}$ and $25^{\circ} \mathrm{C}$. Measurements of p50 were performed at 1, 2, 4, 7, 24, and $48 \mathrm{~h}$ after collection.

Reference values were established on whole blood samples from healthy patients received in our laboratory (LHUB-ULB, $\mathrm{n}=33)$.

\section{Results}

The system has showed a good precision (repeatability CV $\leq 1.04 \%$, intermediate fidelity $\mathrm{CV} \leq 1.90 \%$ ). 
In healthy volunteer, the p50 (mean value: $24.1 \mathrm{mmHg}$ ) is stable up to $7 \mathrm{~h}$ after sampling at $25^{\circ} \mathrm{C}\left(\mathrm{CV}=2.25 \%\right.$ ) and at $4^{\circ} \mathrm{C}$ $(\mathrm{CV}=0.63 \%)$. In the anemic patient $(\mathrm{p} 50=27.9 \mathrm{mmHg})$ and in the newborn ( $\mathrm{p} 50=13.5 \mathrm{mmHg}), \mathrm{p} 50$ was also stable $7 \mathrm{~h}$ after sampling at $4^{\circ} \mathrm{C}(\mathrm{CV}<1.4 \%)$ and at $25^{\circ} \mathrm{C}(\mathrm{CV}<1.39 \%)$.

The measurement range was 23.09 to $28.05 \mathrm{mmHg}$ (mean: $25.35 \mathrm{mmHg}$ ) in healthy adults (20-68 years).

\section{Conclusion}

There is currently a growing interest in the constitutional causes of polycythemia and in particular for high oxygen affinity.

The HEMOX-Analyzer is a simple, fast (1h) and reliable instrument to record the oxyhemoglobin dissociation curve which allows the detection of hemoglobin variants with modified affinity for. The analysis can be performed up to 7 hours after sampling and stored at $4^{\circ} \mathrm{C}$.

\section{C33}

\section{CLINICAL DECISION GUIDELINES FOR MONOCLONAL GAMMOPATHIES REQUIRE AN ASSAY SPECIFIC APPROACH}

$\underline{\text { L. Nevejan }^{1}}$, D. De Smet ${ }^{2}$, M. Vercammen ${ }^{3}$, M. Cornette ${ }^{2}$, P. J. Briers ${ }^{4}$, D. Deeren ${ }^{5}$, J. van Droogenbroeck ${ }^{6}$, K. Fostier ${ }^{7}$, L. van Hoovels $^{8}$

${ }^{1}$ Department of Laboratory Medicine, OLV Hospital, Aalst, Belgium \& Department of Laboratory Medicine, University Hospitals Leuven, Leuven, Belgium, ${ }^{2}$ Department of Laboratory Medicine, AZ Delta Hospital, Roeselare, Belgium, ${ }^{3}$ Department of Laboratory Medicine, Sint-Jan Hospital, Brugge/Oostende \& Research Group REIM Vrije Univesiteit Brussel (VUB), Brussel, Belgium, ${ }^{4}$ Department of Laboratory Medicine, Sint-Jan Hospital, Brugge/Oostende \& Department of Laboratory Medicine, University Hospitals Leuven, Leuven, Belgium, ${ }^{5}$ Department of Hematology, AZ Delta Hospital, Roeselare, Belgium, ${ }^{6}$ Department of Hematology, Sint-Jan Hospital, Brugge/Oostende, Belgium, ${ }^{7}$ Department of Hematology, OLV Hospital, Aalst, Belgium, ${ }^{8}$ Department of Laboratory Medicine, OLV Hospital, Aalst, Belgium \& Department of Microbiology, Immunology \& Transplantation, KU Leuven, Leuven, Belgium

\section{Introduction}

Serum free light chain (sFLC) assays add value to diagnosis, prognosis, and follow-up of patients with plasma cell disorders. Inherent analytical limitations necessitate an extensive verification of newly certified sFLC assays.

\section{Materials and Methods}

Five polyclonal sFLC assay applications were evaluated: Diazyme (Roche) on Cobas c501 and on c503 (Roche), Freelite (TBS) on Optilite (TBS) and on Cobas c501 and Sebia FLC ELISA (Sebia) on AP22 ELITE (DAS). For every assay application, reference value verification was assessed on healthy individuals $(\mathrm{n}=30)$ and patients with chronic kidney disease $(n=60)$. Diagnostic performance was compared on a retrospective selection of 120 serum samples and on follow-up samples of 5 patients with $\kappa$ and $\lambda$ monoclonal gammopathy. Regarding follow-up, the evolution of the involved FLC (iFLC), uninvolved FLC (uFLC), difference iFLC-uFLC (dFLC) and ratio (iFLC/uFLC) for every assay application was interpreted in relation to the clinical evolution.

\section{Results}

sFLC reference values revealed to be application specific and were inversely correlated with glomerular filtration rate.

In concordance to Freelite Optilite, Cohen's $\kappa$ values for Freelite c501 and Diazyme c501/c503 were very good (0.95-0.98) for $\kappa$ sFLC but only moderate for $\lambda$ sFLC (0.78-0.80). For Sebia sFLC ELISA only moderate to good Cohen's kappa for $\kappa \mathrm{sFLC}(0.76), \lambda \mathrm{sFLC}(0.83)$ and $\kappa / \lambda$ ratio (0.84) were obtained. 
In 6/10 follow-up patients, the same kinetics of $\kappa$ and $\lambda$ sFLC were observed between the various SFLC assay applications. For 2 patients, Diazyme FLC on Cobas c503 and Sebia FLC ELISA failed to detect a $\kappa$ resp. $\lambda$ sFLC antigen excess in the diagnostic sample. For the 2 other patients, disease criteria for progressive disease (increase of $\mathrm{dFLC} \geq 100 \mathrm{mg} / \mathrm{L}$ ) were not reliable for non-Freelite assays.

\section{Conclusion}

Differences in reference values and diagnostic performance hamper interchangeability of sFLC results. Assay specific sFLC clinical decision guidelines are warranted.

\section{C34}

\section{DETECTION OF MACROENZYMES: ESTABLISHING REFERENCE INTERVALS FOR EIGHT ENZYMES AFTER PEG PRECIPITATION}

\section{Dedeene $^{1}$, P. Vermeersch ${ }^{1}$, G. Frans ${ }^{1}$}

${ }^{1}$ Clinical Department of Laboratory Medicine, University Hospitals Leuven, Leuven, Belgium

\section{Objectives}

The presence of macroenzymes in blood can significantly hamper clinical diagnoses. To implement a screening for macroenzymes, we established reference intervals and imprecision measures for eight enzymes after PEG-precipitation.

\section{Material and methods}

PEG precipitation was adapted from a published protocol ${ }^{1}$. Per enzyme, at least 40 pseudonymized left-over samples from adult patients were diluted 1:1 with 25\% PEG 6000 and 1:1 with $0.9 \% \mathrm{NaCl}$. Mixtures were incubated for $10 \mathrm{~min}$ at $37^{\circ} \mathrm{C}$ and centrifuged. Supernatant enzyme activity was measured on Cobas c702 and the \% PEG-precipitable activity (\% PPA) was calculated: $\%$ PAA $=100 \times\left[\frac{\left(\text { Activity }_{\mathrm{NaCC}}-\mathrm{Activity}_{\mathrm{PEG}}\right)}{\left.\text { (Activityt }_{\mathrm{NaCl}}\right)}\right]$. After outlier exclusion (Dixon's test), an upper reference limit was calculated for each enzyme $\left(97.5^{\text {th }}\right.$ percentile). Imprecision was determined using a plasmapool with 5 measurements/day for 5 days (CLSI EP-15).

\section{Results}

Results are presented in Table 1. Our intervals were in line with a previous study ${ }^{2}$. Using these intervals, we already identified one patient with macroamylase (77.8\% PPA) and one with macrolipase (98.5\% PPA).

Table 1:

\begin{tabular}{|c|c|c|c|c|c|c|}
\hline \multirow[t]{2}{*}{ Enzyme } & \multicolumn{4}{|c|}{ Upper reference limit (\% PPA) } & \multicolumn{2}{|c|}{ Imprecision PEG precipitation* } \\
\hline & $\begin{array}{r}\text { Samples } \\
(- \\
\text { outliers) }\end{array}$ & $\begin{array}{r}\text { Initial concentration (U/L, median } \\
\text { and range) }\end{array}$ & $\begin{array}{r}\text { UZ } \\
\text { Leuven }\end{array}$ & $\begin{array}{l}\text { Davidson et al } \\
2\end{array}$ & $\begin{array}{r}\text { Inital concentration plasma } \\
\text { pool (U/L) }\end{array}$ & $\begin{array}{r}\text { Within lab } \\
\text { imprecision (\%) }\end{array}$ \\
\hline Amylase & 41 & $106(18-1818)$ & 26 & 60 & 114 & 3.1 \\
\hline ALP & 55 & $93(28-1550)$ & 34 & 36 & 442 & 2.9 \\
\hline ALT & 56 & $27.4(9.9-2506.0)$ & 67 & 76 & 203.8 & 4.9 \\
\hline AST & 56 & $43.8(12.6-10314.0)$ & 51 & 53 & 254.1 & 2.4 \\
\hline $\mathrm{CK}$ & 39 & $227(32-9563)$ & 25 & 37 & 322 & 3.3 \\
\hline GGT & 54 & $61.7(11.1-2796.9)$ & 67 & 51 & 321.6 & 2.5 \\
\hline $\mathrm{LDH}$ & 42 & $263.5(121-4295)$ & 69 & 70 & 454 & 2.5 \\
\hline Lipase & 40 & $86.4(7.5-1917.5)$ & 58 & Not evaluated & 82.1 & 9.0 \\
\hline
\end{tabular}

Evaluated on absolute enzyme concentrations after PEG-precipitation 


\title{
Conclusions
}

We established in-house reference intervals for PEG precipitation of eight enzymes on Cobas c702. This PEG-protocol will be implemented in our core laboratory.

\section{References}

1. 1.Levitt MD, Ellis C. Gastroenterology. 1982;83(2):378-382.

2. Davidson DF, Watson DJM. Ann Clin Biochem. 2003;40(5):514-520.

\section{C35}

\section{COMPARISON OF THREE DIFFERENT METHODS FOR THE QUANTIFICATION OF SERUM AND PLASMA N-TERMINAL PROPEPTIDE OF TYPE III PROCOLLAGEN (PIIINP)}

\author{
$\underline{\text { S. Cugnata }}^{1}$, S. Kovacs ${ }^{2}$, A.-C. Bekaert ${ }^{2}$, F. Watar ${ }^{2}$, J. Demeuse ${ }^{1}$, E. Cavalier ${ }^{3}$ \\ ${ }^{1}$ Department of Clinical Chemistry, University of Liege, Liège, Belgium, ${ }^{2}$ Department of Clinical Chemistry, CHU de Liège, \\ Liège, Belgium, ${ }^{3}$ Department of Clinical Chemistry, University of Liege, CHU de Liège, Liège, Belgium

\section{Background}

The amino terminal propeptide of type III procollagen (PIIINP) is a promising biomarker for sarcopenia and an indicator of liver fibrosis and cirrhosis. The first available method for its quantification was a radioimmunoassay (RIA) produced by Orion but non-radioactive methods are now available. No comparison of these methods has so far been performed.

\section{Objective}

We compared the competitive Orion (Aydan Oy) RIA UniQ PIIINP assay with 2 non-competitive methods, Siemens ADVIA Centaur and Cisbio ELISA to evaluate the harmonization of the assays. We also evaluated the commutability of the assays on EDTA plasma and serum.

\section{Method}

Serum and EDTA plasma samples from sarcopenic patients were collected $(n=25)$, between May and June 2021. Both EDTA plasma and serum PIIINP concentrations were measured with the 3 methods. Bland-Altman plots, Passing Bablok regressions and correlation analyses were used to compare the results $(\mu \mathrm{g} / \mathrm{L})$.

\section{Result}

There was a poor agreement between RIA and other methods: Centaur $=3,33$ [95\%CI: 2,33; 4,41] $\times$ RIA - 6,44 [95\%CI: $-13,55$ to $-4,47]$ in serum $(r=0,20)$ and Centaur $=3,89[3,36 ; 5,05] \times$ RIA $-7,31[-11,80 ;-4,85]$ in plasma $(r=0,20)$. ELISA $=4,36[2,63 ; 10,70]$ $\times$ RIA - 12,36 [-43,46; -4,40 ] in serum $(r=0,15)$ and ELISA $=4,30[2,92 ; 6,68] \times \operatorname{RIA}-8,18[-16,50 ;-3,48]$ in plasma $(r=0,17) . A$ much better concordance was observed between Centaur and ELISA: Centaur $=0.82[0,48 ; 1,40] \times$ ELISA $+1.76[-3,15 ; 4,74]$ in serum $(r=0,62)$ and Centaur $=1,00[0,59 ; 1,44] \times$ ELISA $+0.23[-3,21 ; 2,59]$ in plasma $(r=0,69)$. Finally, the regression equations between serum and plasma were Centaur (plasma) $=0,88[0,80$ to 0,96$] \times$ Centaur(serum) $-0,63[-1,59 ;-0,006](\mathrm{r}=0,88)$ and ELISA $($ plasma $)=0,83[0,65 ; 1,01] \times$ ELISA $($ serum $)+0,15[-2,09 ; 2,04](\mathrm{r}=0,88)$.

\section{Conclusion}

Orion (Aidan Oy) UniQ PIIINP RIA assay presented significantly discrepant results compared to Siemens ADVIA Centaur and Cisbio ELISA. The reason could be linked to a lack of specificity of the RIA which probably cross-reacts with proPIIINP. ADVIA Centaur and ELISA methods are correlated and suitable for the PIIINP detection. However, serum and plasma present significantly different results with ADVIA Centaur and serum should be preferred. 


\title{
C36
}

\section{DEVELOPMENT OF A MASS SPECTROMETRY METHOD FOR THE THERAPEUTIC DRUG MONITORING OF NINE ANTITUBERCULOSIS DRUGS}

\author{
D. Fage $^{1}$, R. Brilleman ${ }^{2}$, G. Deprez ${ }^{1}$, F. Cotton ${ }^{1}$ \\ ${ }^{1}$ Department of Clinical Chemistry, LHUB-ULB, Université Libre de Bruxelles, Brussel, Belgium, ${ }^{2}$ Student in Pharma- \\ ceutical Sciences ULB, Brussel, Belgium
}

\section{Objective}

Development of a measurement method to perform the therapeutic drug monitoring (TDM) of the main anti-tuberculosis drugs (anti-TB) used in the treatment of multi-S and multi-R tuberculosis.

\section{Material and Methods}

The 9 drugs included in the panel were the 4 first-line drugs (ethambutol [EMB], isoniazid [INH], pyrazinamide [PZA] and rifampicin [RMP]), and 5 second-line drugs (levofloxacin [LFX], linezolid (LZD], moxifloxacin [MFX], rifabutin [RFB]) and bedaquiline [BDQ]). An isotopic standard was used for each compound. Acetyl-INH [Ac-INH], the main metabolite of INH, was also quantified to determine the acetylator phenotype of the patient.

Briefly, to $200 \mu \mathrm{L}$ of plasma sample were added $50 \mu \mathrm{L}$ of internal standards mix and $800 \mu \mathrm{L}$ of acetonitrile. Eight hundred $\mu \mathrm{L}$ of supernatant were recovered, evaporated under nitrogen at $40^{\circ} \mathrm{C}$ and reconstituted with $100 \mu \mathrm{L}$ of mobile phase. Ten $\mu \mathrm{L}$ were injected into a LC-MS/MS system from Agilent Technologies. The chromatographic separation was performed within $10 \mathrm{~min}$ and was followed with a post-run of $5 \mathrm{~min}$. Calibrators and controls were home-made preparations and were extracted with the same protocol.

The measurement range was adapted for each drug to monitor the plasma concentration at $2 \mathrm{~h}$ (peak) and $6 \mathrm{~h}$ to evaluate the enteric absorption. The accuracy profiles approach was used to validate the method. The acceptance limits were fixed at $\pm 30 \%$ and the alpha risk was set at $5 \%$.

\section{Results}

The validated ranges were the following: EMB/INH/Ac-INH/LFX: 0.1-20 mg/L; RMP/LZD: 1-50 mg/L; PZA: 1-100 mg/L; MFX: $0.01-20 \mathrm{mg} / \mathrm{L}$; RFB: $0.25-5 \mathrm{mg} / \mathrm{L}$ and BDQ: $0.05-10 \mathrm{mg} / \mathrm{L}$. The intermediate precision (CV) and the trueness were below $10 \%$ and $11 \%$, respectively.

\section{Conclusion}

The developed method allowed the quantification of the main anti-TB used in clinical practice with adapted ranges of measurement. To our knowledge, this is the first method including bedaquiline in anti-TB panel and the first one using isotopic standards for each compound.

\section{C37}

\section{MASSIVE BACLOFEN SELF-POISONING IN A 16 YEARS-OLD TEENAGER: A CASE REPORT}

\author{
$\underline{\text { A. Nizet }}^{1}$, R. Denooz ${ }^{1}$, C. Charlier ${ }^{1}$ \\ ${ }^{1}$ Laboratory of Clinical, Forensic and Environmental Toxicology, Center for Interdisciplinary Research on Medicines \\ (CIRM), University Hospital of Liege, Liege, Belgium

\section{Objectives}

Baclofen, a selective agonist of the GABA(B) receptor, is originally used as treatment for central spasticity and, occasionally to treat alcohol addiction [1]. However, severe baclofen poisoning can result in severe respiratory depression, 
autonomic disturbances, seizures, and coma, requiring intensive supportive care to avoid death [2]. Here we reported a 16-year-old girl who was found unresponsive by her father at home. At admission in emergency department, clinical examination showed respiratory acidosis ( $\mathrm{pH} 7.17$ ) and hypotension (101/53 mmHg). Her Glasgow Coma Score was 3, pupils were fixed dilated with no response to light, and she had no spontaneous movements. For optimal care, the patient was sedated, intubated, and ventilated. Following these symptoms, baclofen poisoning was considered.

The aim of the project was to research and quantify baclofen in patient blood and establish elimination kinetics of the drug.

\title{
Material and methods
}

Successive determinations of baclofen in patient blood were made at different times since admission at emergency unit. For assaying baclofen, samples were centrifugated, deuterated baclofen internal standard was added and precipitated by acidified acetonitrile. After nitrogen evaporation of supernatant, samples were diluted by acidified mix of water/methanol (90/10: v/v) solution and analyzed by LC-MS/MS (UHPLC Acquity®, Waters).

\section{Results}

Baclofen blood concentration was defined as therapeutic or "normal" between 0.08 to $0.6 \mathrm{mg} / \mathrm{L}$, toxic from 1.1 to $3.5 \mathrm{mg} / \mathrm{L}$ and comatose-fatal from 6 to $9.6 \mathrm{mg} / \mathrm{L}$ [3]. Baclofen concentration in patient blood at admission was $12.507 \mathrm{mg} / \mathrm{L}$, higher than the literature comatose-fatale concentration. In addition, blood toxicological screening demonstrated toxic concentration of trazodone $(4.5 \mathrm{mg} / \mathrm{L}$ ) simultaneously. Ninety-eight hours after admission, baclofen blood concentration was in therapeutic range $(0.437 \mathrm{mg} / \mathrm{L})$.

\section{Conclusion}

Baclofen intoxication can lead to the death of the patient without an intensive care including both respiratory and hemodynamic support. Laboratory results are essential to detect and quantitate possible drug intoxications and allow clinicians to provide appropriate care. In this case, intensive supportive care was vital for the patient. Finally, therapeutic blood concentration of baclofen was obtained after 98 hours. This corresponding to the moment of improvement of the patient condition.

\section{References}

[1] F. Pelissier, L. de Haro, F. Cardona, C. Picot, E. Puskarczyk, J.M. Sapori, C. Tournoud, N. Franchitto, Self-poisoning with baclofen in alcohol-dependent patients: national reports to French Poison Control Centers, 2008-2013, Clin. Toxicol. 55 (2017) 275-284. https://doi.org/10.1080/15563650.2017.1284330.

[2] R. Sullivan, M.J. Hodgman, L. Kao, L.M. Tormoehlen, Baclofen overdose mimicking brain death, Clin. Toxicol. 50 (2012) 141-144. https://doi.org/10.3109/15563650.2011.654209.

[3] F.P. Meyer, Indicative therapeutic and toxic drug concentrations in plasma: a tabulation., Int. J. Clin. Pharmacol. Ther. 32 (1994) 71-81. http://www.ncbi.nlm.nih.gov/pubmed/8004362.

\section{C38}

\section{STABILITY STUDY OF PSYCHOTROPIC DRUGS IN SALIVA}

\author{
$\underline{\text { A. Nizet }}^{1}$, N. Dubois ${ }^{1}$, E. Hilligsmann ${ }^{1}$, C. Charlier ${ }^{1}$
}

${ }^{1}$ Laboratory of Clinical, Forensic and Environmental Toxicology, Center for Interdisciplinary Research on Medicines (CIRM), University Hospital of Liege, Belgium., Liege, Belgium

\section{Objectives}

According to the Belgian Royal Decree (B.R.D.), saliva can be used for the quantification of psychotropic drugs in the context of driving under the influence (DUI) of drugs (B.R.D. 30 November $2015-1^{\text {st }}$ Chapter, $1^{\text {st }}$ section: article 5 and article 7). After collection by the Police, samples must be stored in a fridge between 2 and $8{ }^{\circ} \mathrm{C}$ until analysis by an 
approved laboratory. After determination, excess samples have to be stored in a freezer at $-18^{\circ} \mathrm{C}$ or below for six months in case of counter-expertise. Then, they will be destroyed.

The aim of the project was to study the stability of $\Delta$-9-tétrahydrocannabinol (THC), 6-monoacetylmorphine (6-MAM), morphine, amphetamine, MDMA, cocaine and benzoylecgonine in saliva after storage in fridge $\left(2-8^{\circ} \mathrm{C}\right)$ and in freezer $\left(-20^{\circ} \mathrm{C}\right)$ during six months, as required by the Belgian Royal Decree.

\title{
Material and methods
}

Saliva samples spiked with THC, 6-MAM, morphine, amphetamine, MDMA, cocaine and benzoylecgonine were stored at $+4^{\circ} \mathrm{C}$ and $-20^{\circ} \mathrm{C}$ after addition of storage buffer during six months. For THC analysis, samples were centrifugated, deuterated THC internal standard was added and extracted by a mixture of hexane/ethyl acetate (9/1: v/v). Supernatants were dried and diluted by methanol/water mix $(80 / 20: \mathrm{v} / \mathrm{v})$. The samples containing the other drugs underwent a solid phase extraction on OASIS ${ }^{\circledR}$ MCX (Waters) cartridges after acidification and addition of deuterated internal standards. Finally, extracted samples were injected onto a UHPLC-MS/MS (UHPLC Acquity ${ }^{\circledR}$, Waters) according to two different analytical methods. Samples were analyzed in quintuplate each week during 4 weeks and each month until the end of the study. Analytes were considered as stable when the bias between the mean concentration of the quintuplates and the mean value obtained at day 0 was lower than $15 \%$.

\section{Results}

At refrigeration conditions, THC (recovery: 88\%) and cocaine (recovery: 88\%) are stable for up to 21 days whereas 6-MAM (recovery: 91\%), morphine (recovery: 113\%), amphetamine (recovery: 104\%) and benzoylecgonine (recovery: 114\%) are stable for up to 28 days. MDMA showed the poorest stability with a recovery of $122 \%$ after only 14 days. At $-20^{\circ} \mathrm{C}$, THC (recovery: 95\%), morphine (recovery: 99\%), cocaine (recovery: 95\%) and benzoylecgonine (recovery: 114\%) are stable for up to 28 days whereas 6-MAM (recovery: 107\%) is stable up to 21 days. Amphetamine (recovery: 70\% after 14 days) and MDMA (recovery: 135\% after 14 days) showed the highest instability.

\section{Conclusion}

This stability study demonstrated that the storage temperature of samples and time before analysis could have an impact on the results that will be returned to the magistrate. Nevertheless, degradation of 6-MAM in morphine and cocaine in benzoylecgonine allows to keep a right interpretation for these molecules whereas it is not the case for THC, amphetamine and MDMA.

\section{C39}

\section{COMBINED FLOWCYTOMETRIC PHENOTYPING AND INTERFERON RELEASE ASSAY OF SARS-COV-2 REACTIVE T CELLS IN WHOLE BLOOD}

\author{
$\underline{\text { B. Calcoen }}^{1}$, K. Callebaut ${ }^{2}$, A. Vandenbulcke ${ }^{1}$, N. Callewaert ${ }^{2}$, X. Bossuyt ${ }^{3}$, K. Vanhoorelbeke ${ }^{1}$, N. Geukens ${ }^{4}$, S. De Meyer ${ }^{1}$, \\ W. Maes ${ }^{4}$ \\ ${ }^{1}$ Laboratory for Thrombosis Research, Kortrijk, Belgium, ${ }^{2} \mathrm{AZ}$ Groeninge Hospital, Kortrijk, Belgium, ${ }^{3}$ Department of \\ Laboratory Medicine, University Hospitals of UZ Leuven, Leuven, Belgium, ${ }^{4}$ PharmAbs, The KU Leuven Antibody Center, \\ KU Leuven, Leuven, Belgium
}

\section{Objectives}

Our aim was to include flowcytometric assessment of SARS-CoV-2 specific T cell activation to a commercially available cytokine release assay on whole blood samples. 


\section{Material and Methods}

The EUROIMMUN SARS-CoV-2 interferon gamma release assay (IGRA) was performed according to the manufacturer's instructions on whole blood samples from donors vaccinated against SARS-CoV-2 $(n=6)$. In addition, the remaining pellet after centrifugation was resuspended in physiological buffer. Reconstituted samples were stained with a panel of fluorescently labeled monoclonal antibodies including anti-CD3, anti-CD4, anti-CD8 and anti-CD69. Following lysis of red blood cells, flowcytometric sample acquisition was performed.

\section{Results}

Specific interferon gamma release (mean $=1368 \pm 937.8 \mathrm{mIU} / \mathrm{mL}$ ) was found in whole blood from vaccinated donors upon stimulation with SARS-CoV-2 antigens. Furthermore, using our protocol, it was possible to reliably differentiate both CD4+ and CD8+ T cells in the reconstituted whole blood samples following IGRA. In addition, expression of the early T cell activation marker CD69 was significantly upregulated in both CD4+ and CD8+ T cells from vaccinated donors upon stimulation with SARS-CoV-2 antigens (Figure 1).

\section{Conclusion}

This study showed that flowcytometric profiling of T cell activation can be successfully implemented as additional readout to a commercially available SARS-CoV-2 specific interferon gamma release assay.

\section{Acknowledgements}

We would like to thank EUROIMMUN Medizinische Labordiagnostika AG and BIOGNOST CV for providing materials used in this study.

A
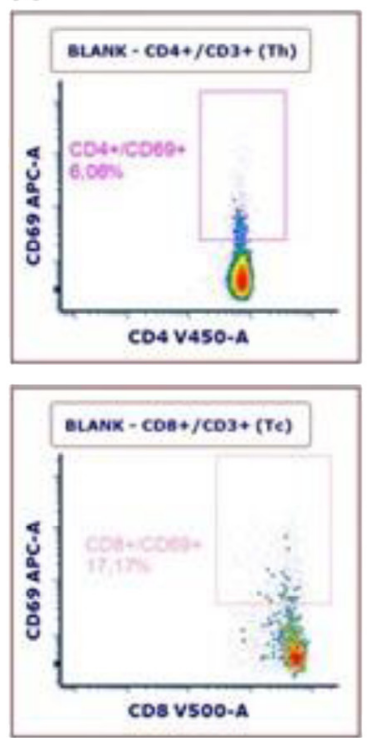
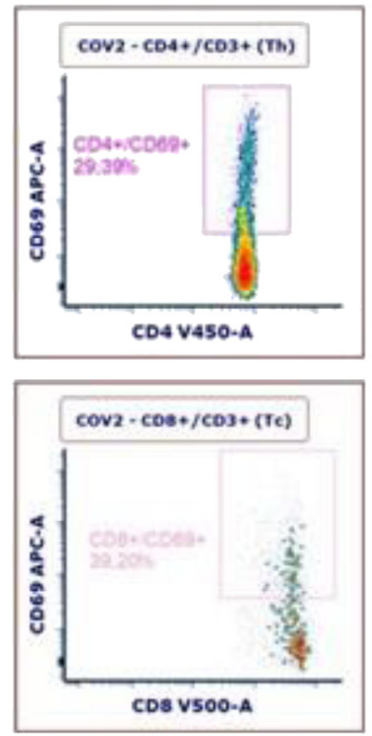

B

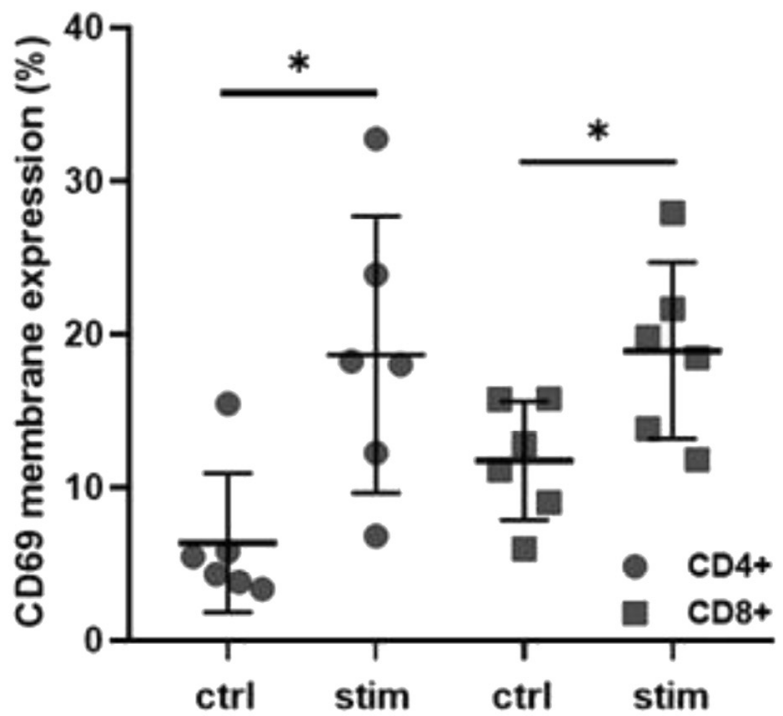

Figure 1: Flowcytometric assessment of SARS-CoV-2 specific T cell activation in whole blood from donors vaccinated against SARS-CoV-2. A) Representative dot plots showing CD69 membrane expression within CD4+ T cells (upper plots) within SARS-CoV-2 antigen stimulated cells (right) compared to the unstimulated control (left). The lower plots show CD69 membrane expression within CD8+ T cells within SARS-CoV-2 antigen stimulated cells (right) compared to the unstimulated control (left). B) Percentage of CD69 membrane expression in CD4+ and CD8+ $T$ cells upon stimulation with SARS-CoV-2 antigens (stim) versus unstimulated controls (ctrl), * $\mathrm{p}<0.05, \mathrm{n}=6$. 


\title{
C40
}

\section{EVALUATION OF HBA1C MEASUREMENT ON HLC-723G8 IN PATIENTS WITH SUSPECTED HEMOGLOBIN RICCARTON VARIANT}

\author{
A. Ockerman ${ }^{1}, \underline{\text { S. Lefever }}{ }^{1}$, M. Tajdar ${ }^{1}$, C. van Laer $^{1}$ \\ ${ }^{1}$ UZ Leuven, 3000, Belgium

\section{Objective}

Hemoglobin (Hb) Riccarton has been described to affect HbA1c measurements on HPLC analyzers. ${ }^{1}$ Assays based on boronate affinity are considered relatively resistant to interference by most hemoglobin variants. We evaluate if HPLC performed with G8 (HLC-723G8, Tosoh Bioscience, Tokyo, Japan) is accurate enough for monitoring HbA1c levels in patients with (suspected) Hb Riccarton.

\section{Material and Methods}

We performed a retrospective evaluation of HbA1c measurements for patients with suspected Hb Riccarton $(n=30)$ and a control group of patients with heterozygote presence of $\mathrm{HbS}, \mathrm{HbC}$ or $\mathrm{HbD}(\mathrm{n}=37)$ knowing not to interfere with HbA1c measurement. HbA1c analysis was performed with G8 and the point-of-care Afinion device (Afinion ${ }^{\mathrm{TM}}$ AS100, Abbott, Illinois, USA). Suspected Hb Riccarton was defined as a visible shoulder to the right of the sA1C fraction on the G8-chromatogram in combination with a broader hemoglobin A fraction on capillary zone electrophoresis (Minicap, Sebia, Paris, France). All G8-chromatograms were inspected for full integration of the shoulder fraction.

\section{Results}

The mean absolute difference of HbA1c assays $(\mathrm{mmol} / \mathrm{mol})$ and correlation coefficient between both methods of Hb1Ac assays ( $\mathrm{mmol} / \mathrm{mol}$ ) are shown in table 1 for different patient groups. These were comparable for the $\mathrm{Hb}$ Riccarton group with full integration of the shoulder fraction and the control group. However, poor correlation was found in samples with only partial integration of the shoulder fraction.

\section{Conclusion}

In patients with suspected Hb Riccarton HLC-723G8 can be used for accurate HbA1c monitoring after visual chromatogram inspection for full integration of the sA1C shoulder. In cases where full integration fails the boronate affinity assay with Afinion has shown to be a useful alternative.

\section{Reference}

1. van den Ouweland JM et al, Clin Chem Lab Med. 2008, PMID: 18601605.

Table 1: Bland-Altman analysis of comparison between $\mathrm{G8}$ and Afinion HbA1c measurement for $\mathrm{Hb}$ Riccarton and control group (Hb AS/AC/AD)

\begin{tabular}{lcccr}
\hline Hemoglobin variant & $\mathbf{n}$ & Mean difference $(\mathbf{m m o l} / \mathbf{m o l})$ & $\mathbf{9 5 \%} \mathbf{C l}$ (mmol/mol) & correlation coefficient \\
\hline Hb Riccarton (all) & 30 & 0.3 & $-6,9$ to 7,6 & 0.967 \\
Full integration & 20 & 2.2 & -2.9 to 7.3 & 0.988 \\
Partial integration & 10 & -3.4 & -8.6 to 1.8 & 0.948 \\
Hb AS/AC/AD & 37 & 1.6 & -5.8 to 9.0 & 0.979 \\
\hline
\end{tabular}




\title{
C41
}

\section{COMPARISON OF ROMA SCORE, HE4 AND CA125 MARKERS IN PRE- AND POSTMENOPAUSAL WOMEN: PRELIMINARY RESULTS OF A 6-YEAR RETROSPECTIVE STUDY IN A TERTIARY REFERRAL HOSPITAL}

\author{
B. Lardinois ${ }^{1}$, A. Randazzo ${ }^{1}$, R. Debois ${ }^{1}$
}

${ }^{1}$ Laboratory Department, CHU UCL Namur Site de Sainte-Elisabeth, Université catholique de Louvain, Namur, Belgium

\section{Background}

Epithelial ovarian cancer is often diagnosed at a late stage thus inducing a poor prognosis. Surgical procedures are often needed to assess the benign or malign nature of the tumor. The diagnostic performance of Ca125 and He4 usual biomarkers or algorithms such as Risk of Ovarian Malignancy Algorithm (ROMA) have been widely assessed in pelvic masses but only few studies retrospectively compared their results to histopathological findings.

\section{Objectives}

To retrospectively determine the best diagnostic accuracy between ROMA score, HE4 and Ca125 using histopathological results in the initial evaluation of adnexal or pelvic mass.

\section{Material and Methods}

We retrospectively collected the ROMA score, HE4, Ca125 and histopathological results of 137 patients who underwent surgery. Histopathological type of benign or malign ovarian tumours were classified according to the World Health Organization classification system. A distinction between premenopausal and postmenopausal women was performed in order to compare the accuracy between the two different groups.

\section{Results}

Receiver operating characteristic curves are shown in Figure 1. In premenopausal women, we obtained an AUC of 0.81 (95\% Confidence Interval (CI) 0.69-0.94; $\mathrm{p}=0.003$ ), 0.81 (95\%CI 0.69-0.93; $\mathrm{p}=0.003$ ) and 0.79 (95\%CI 0.630.96; $\mathrm{p}=0.005)$ for ROMA score, HE4 and Ca125, respectively. The AUC were higher for postmenopausal women : 0.95 (95\%CI 0.92-0.99; $\mathrm{p}<0.0001), 0.93$ (95\%CI 0.89-0.97; $\mathrm{p}<0.0001)$ and 0.90 (95\%CI 0.84-0.96; $\mathrm{p}<0.0001)$, respectively.

Premenopausal

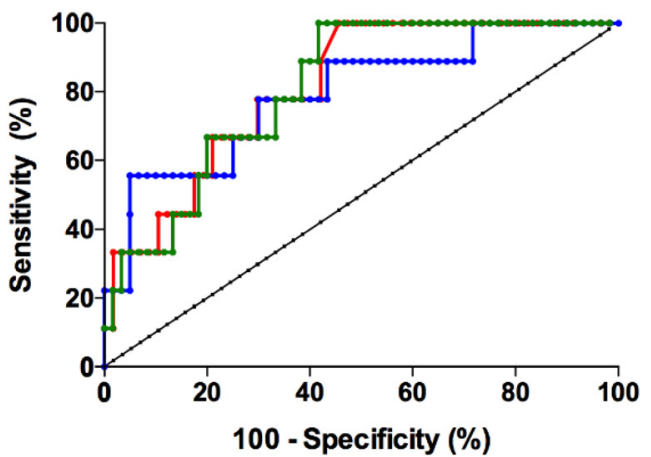

Postmenopausal

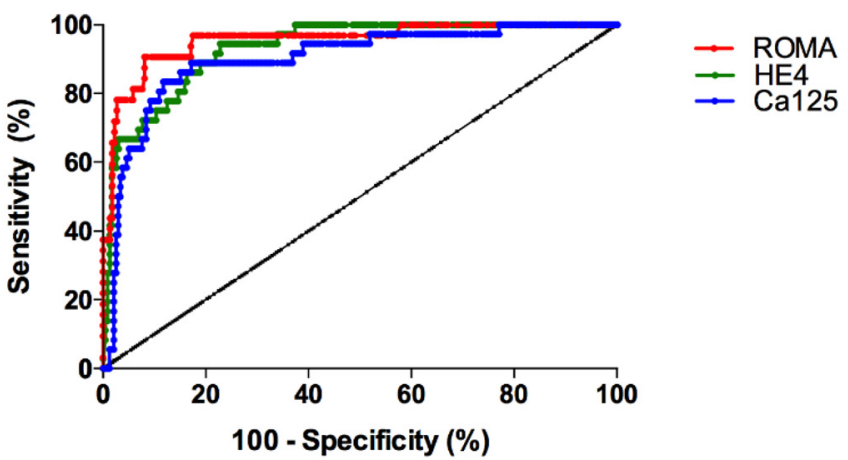

Figure 1: Receiver operating characteristic curves of benign vs. malignant ovarian tumours in premenopausal (left graph) and postmenopausal (right graph) women. 


\title{
Conclusions
}

ROMA score is the best criterion for differentiating benign and malignant ovarian masses in postmenopausal women with a greater diagnostic accuracy and a higher sensitivity compared to usual biomarkers. The diagnostic performance of ROMA score, He4 and Ca125 is comparable in premenopausal women.

\section{M1}

\section{COMPARISON OF THE LIAISON SARS-COV-2 TRIMERICS IGG ASSAY BY DIASORIN AND THE COBAS ELECSYS SARS-COV-2 S IGG ASSAY BY ROCHE}

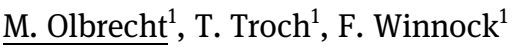 \\ ${ }^{1}$ Algemeen Stedelijk Ziekenhuis (ASZ), Aalst, Belgium

\section{Objective}

While molecular testing is still the gold standard for the diagnosis of Coronavirus disease-19 (COVID-19), caused by severe acute respiratory syndrome coronavirus (SARS-CoV-2), serological testing can be used to detect the patient's adaptive immune response to recent and past infections. To use serological tests properly, it is important to understand their performance characteristics and limitations.

\section{Material and Methods}

We evaluated 2 serological assays. The Liaison SARS-CoV-2 TrimericS IgG assay by DiaSorin is an indirect chemiluminescence immunoassay (CLIA) for the quantitative determination of anti-trimeric spike protein specific IgG antibodies. The Cobas CLIA Elecsys SARS-CoV-2 S assay by Roche measures antibodies (including IgG) to SARS-CoV-2 spike protein receptor binding domain quantitatively.

55 human serum samples are used to assess the performance of the tests. The diagnosis of all patients was confirmed by RT-PCR or positive vaccination status ( $\geq 14$ days after vaccination).

\section{Results}

We found a total coefficient of variation of 5,32\% and 1,77\%, a total error of $11,18 \%$ and $15,56 \%$ for respectively the DiaSorin assay and the Roche assay, after measuring the positive control for 8 days with 4 repeated measurements a day.

The specificity for both IgG assays was 100\%. The Roche assay resulted in a higher sensitivity (95,65\%) in comparison to the DiaSorin assay (82,61\%). We found 6 discordant pairs, 1 after infection and 5 early after vaccination. All were positive for the Roche assay and negative for the DiaSorin assay. The p-value equals 0,0412 with a McNemar test. By conventional criteria $(\mathrm{p}<0,05)$, this difference is considered to be statistically significant.

\section{Conclusion}

Both automated serological assays for detecting SARS-CoV-2 antibodies in COVID-19 are comparable for imprecision and total error. They are highly specific but vary in sensitivity. McNemar test showed a significant difference $(\mathrm{p}<0,05)$ between the 2 assays. Further studies with larger sample sizes are needed to confirm these findings. 
M2

\title{
VALIDATION OF COLIBRÍ FOR AUTOMATED PREPARATION OF MALDI-TOF TARGETS FOR YEAST IDENTIFICATION
}

\author{
$\underline{\text { R. Heestermans }}{ }^{1}$, P. Herroelen ${ }^{1}$, K. Emmerechts ${ }^{1}$, K. Vandoorslaer ${ }^{1}$, I. Wybo ${ }^{1}$, D. Piérard ${ }^{1}$, A. Muyldermans ${ }^{1}$ \\ ${ }^{1}$ Universitair Ziekenhuis Brussel, Vrije Universiteit Brussel (VUB), Brussel, Belgium
}

\section{Objective}

Recently, Copan (Italy) introduced the Colibrî ${ }^{\mathrm{TM}}$ for automated colony picking and preparation of MALDI-TOF target plates. Our study aimed to validate this system for yeasts as this has not been performed yet and is a missing link to implement it for routine use.

\section{Material and Methods}

Fifty Candida strains were selected to evaluate accuracy of Colibri ${ }^{\mathrm{TM}}$ : C. albicans $(\mathrm{n}=7)$, . glabrata $(\mathrm{n}=9)$, C. parapsilosis $(\mathrm{n}=7)$, C. tropicalis $(\mathrm{n}=8)$, C. krusei $(\mathrm{n}=2)$, C. guilliermondii $(\mathrm{n}=6)$, C. dubliniensis $(\mathrm{n}=9)$ and C. auris $(\mathrm{n}=2)$. For each strain, a Sheep Blood Agar plate supplemented with X and V factors (HEM) and a Sabouraud Agar plate (SAB) were inoculated and incubated by WASPlab® (Copan). After $18 \mathrm{~h}$ and $36 \mathrm{~h}$ of incubation, isolates were spotted in parallel by Colibri ${ }^{\mathrm{TM}}$ and manually from each culture plate onto a MALDI target plate with addition of formic acid. Acceptance criterium for identification (ID) by MALDI Biotyper ${ }^{\circledR}$ (Bruker, Germany) was set on 1.8 .

\section{Results}

Sufficient growth after $18 \mathrm{~h}$ incubation was observed for 36/50 HEM and 44/50 SAB plates. Overall, using Colibri ${ }^{\mathrm{TM}}$ for colony picking, $86 \%$ of strains cultured on HEM plates were identified with an acceptable ID score compared to $81 \%$ manually. SAB plates showed inferior results for both Colibri ${ }^{\mathrm{TM}}(66 \%)$ and manually $(61 \%)$. When evaluating the accuracy of Colibri $^{\mathrm{TM}}$, there was an overall agreement of $86 \%$ for identification of strains on HEM plates between Colibri $^{\mathrm{TM}}$ and the manual method after $18 \mathrm{~h}$ and $84 \%$ after $36 \mathrm{~h}$. For SAB plates, an agreement of $77 \%$ after $18 \mathrm{~h}$ and $89 \%$ after $36 \mathrm{~h}$ was observed. With exception of $C$. dubliniensis and $C$. tropicalis, all included Candida species showed a $100 \%$ accuracy for Colibrî ${ }^{\mathrm{TM}}$ on HEM plates.

\section{Conclusion}

We observed a good agreement between Colibrí ${ }^{\mathrm{TM}}$ and the manual reference method. These results demonstrate that Colibri $^{\mathrm{TM}}$ is a reliable system for MALDI-TOF target preparation for yeast identification, allowing an increase in standardization and lower hands-on time.

\section{M3}

\section{VALIDATION OF RAPID ANTIMICROBIAL SUSCEPTIBILITY TESTING USING WASPLAB®}

\author{
$\underline{\text { P. Herroelen }^{1}, \text { R. Heestermans }}{ }^{1}$, K. Emmerechts ${ }^{1}$, K. Vandoorslaer ${ }^{1}$, I. Wybo ${ }^{1}$, D. Piérard ${ }^{1}$, A. Muyldermans ${ }^{1}$
}

${ }^{1}$ UZ Brussel, Jette, Belgium

\section{Introduction}

With the increase in antimicrobial resistance, fast reporting of antimicrobial susceptibility testing (AST) results is becoming increasingly important. EUCAST developed a method for rapid AST (RAST) directly from the broth of positive blood cultures (BC) with reading of inhibition zones at 4, 6 and 8 hours. 


\section{Methods}

We evaluated the RAST method based on EUCAST disc diffusion methodology with inoculation of BC broth using Copan WASPLab ${ }^{\circledR}$ (inclusive Colibrí ${ }^{\mathrm{TM}}$ and Radian $^{\circledR}$ ). 49 non-duplicate strains were tested: $E$. coli $\mathrm{n}=17$, $K$. pneumoniae $\mathrm{n}=7, P$. aeruginos $\mathrm{n}=4$, . baumannii $\mathrm{n}=2$, S. aureus $\mathrm{n}=10$, E. faecalis $\mathrm{n}=6$ and $E$. faecium $\mathrm{n}=3$. Results were compared to direct AST (DAST; performed manually) and standardized AST (using Copan Colibrí ${ }^{\mathrm{TM}}$ ). Categorical agreement (CA) was calculated, based on standardized AST as reference method. $\chi^{2}$ test was used for comparing proportions of categorical variables.

\section{Results}

Good CA was obtained at all time points for all groups, except $P$. aeruginosa (6h, 87.5\%; 8h, 84.2\%). No significant difference in CA was observed between RAST and DAST. RAST cut-offs for extended-spectrum $\beta$-lactamase (ESBL)producing Enterobacterales enabled the detection of all included ESBL isolates $(\mathrm{n}=5)$ at all time points, except for 1 $E$. coli at 4h. RAST cut-offs for carbapenemase-producing Enterobacterales (CPE) enabled the detection of only one carbapenemase at $6 \mathrm{~h}$. However, all carbapenemases $(n=3)$ were correctly detected at $8 \mathrm{~h}$. Two methicillin resistant S. aureus (MRSA) were included; both were correctly categorized as cefoxitin resistant at $6 \mathrm{~h}$ and $8 \mathrm{~h}$. At $4 \mathrm{~h}$, there was insufficient growth for inhibition zone interpretation.

\section{Conclusions}

EUCAST RAST provides a fast and reliable alternative for standardized AST of positive BC. The fully automated WAS$\mathrm{PLab}^{\circledR}$ provides a significant advantage as pictures are made automatically implicating that we are not strictly bound to the time points for inhibition zone interpretation.

\section{M4}

\section{TWO SEPARATE CLUSTERS OF SARS-COV-2 DELTA VARIANT INFECTIONS IN A SINGLE GROUP OF 41 STUDENTS TRAVELLING FROM INDIA TO BELGIUM: AN ILLUSTRATION OF THE NEED FOR RIGOROUS TESTING AND QUARANTINE}

$\underline{\text { J. van Elslande }}{ }^{1}$, F. Kerckhofs ${ }^{1}$, L. Cuypers ${ }^{1}$, A. Vankeerberghen ${ }^{2}$, E. Wollants ${ }^{3}$, B. Potter ${ }^{4}$, L. Cattoir ${ }^{2}$, A. Holderbeke ${ }^{2}$, S. Behillil ${ }^{5}$, S. Gorissen ${ }^{1}$, M. Bloemen ${ }^{3}$, J. Arnout ${ }^{6}$, M. van Ranst ${ }^{1}$, J. van Weyenbergh ${ }^{7}$, P. Maes $^{3}$, G. Baele $^{4}$, P. Vermeersch $^{1}$, E. André

${ }^{1}$ University Hospitals Leuven, Clinical Department of Laboratory Medicine, Leuven, Belgium, ${ }^{2}$ Laboratory of Clinical Microbiology, OLV Hospital Aalst, Aalst, Belgium, ${ }^{3} \mathrm{KU}$ Leuven, Rega Institute, Department of Microbiology, Immunology and Transplantation, Laboratory of Clinical and Epidemiological Virology, Leuven, Belgium, ${ }^{4} \mathrm{KU}$ Leuven, Rega Institute, Department of Microbiology, Immunology and Transplantation, Laboratory of Clinical and Evolutionary Virology, Leuven, Belgium, ${ }^{5}$ Molecular Genetics of RNA viruses, CNRS UMR 3569, Université de Paris, Institut Pasteur, Paris, France, ${ }^{6} \mathrm{KU}$ Leuven, Biomedical Sciences group management, Leuven, Belgium, Leuven, Belgium, ${ }^{7} \mathrm{KU}$ Leuven, Rega Institute, Department of Microbiology, Immunology and Transplantation, Laboratory of Clinical Bacteriology and Mycology, Leuven Belgium, Leuven, Belgium

\section{Objectives}

The emergence of variants of concern with higher transmissibility and partial immune escape poses an additional challenge for travel-related control measures to mitigate the spread of SARS-CoV-2 variants via international travel. Only limited real-life data are available regarding variant-specific measures, with most of previous evidence derived from modelling studies. This study aimed to provide real-life data on the effectiveness of travel control measures in Belgium against importation of the SARS-CoV-2 B.1.617.2 (Delta) variant. 


\section{Materials and Methods}

We extensively characterized two clusters of SARS-CoV-2 Delta variant infections in a group of 41 Indian nursing students who travelled from New Delhi, India, to Belgium via Paris, France. The students underwent repeated RT-PCR testing, subgenomic RNA PCR, viral whole genome sequencing with phylogenetic analysis and antibody testing.

\section{Results}

All students tested negative before departure and had a second negative antigen test upon arrival in Paris. Upon arrival in Belgium, the students were quarantined in 8 different houses for 10 days, with a planned RT-PCR test on day 7 . At the time of the first PCR test, everybody was still asymptomatic. Four houses remained COVID-free, while all residents of the other four houses developed an infection during quarantine including 4 residents who were fully vaccinated and the 2 residents who were partially vaccinated. Viral whole genome sequencing revealed two distinct strains, affecting respectively one and three houses. Vaccination status did not seem to prevent infection nor decrease the viral load. No severe symptoms were reported. Extensive contact tracing and 3 months of nationwide genomic surveillance confirmed that these outbreaks were successfully contained and did not contribute to onward community transmission in Belgium.

\section{Conclusions}

These clusters confirm the importance of pre-travel testing, quarantine of $\geq 7$ days upon arrival and quarantine exit testing regardless of vaccination status or symptoms to mitigate the risk of import of SARS-CoV-2 variants. This is especially relevant for travelers coming from countries experiencing a surge of infections with a new variant of concern.

\section{M5}

\section{ESTIMATED AVERAGE TIME TO SERONEGATIVITY FOR SARS-COV-2 ANTI-SPIKE MORE THAN DOUBLE THAT OF ANTI-NUCLEOCAPSID ANTIBODIES IN COVID-19 PATIENTS}

J. van Elslande ${ }^{1}$, M. Oyaert ${ }^{2}$, N. Lorent ${ }^{3}$, Y. Vande Weygaerde ${ }^{4}$, L. Godderis ${ }^{5}$, M. van Ranst ${ }^{1}$, E. André ${ }^{1}$, E. Padalko ${ }^{2}$, K. Lagrou ${ }^{1}$, S. Vandendriessche ${ }^{2}$, P. Vermeersch ${ }^{1}$

${ }^{1}$ University Hospitals Leuven, Clinical Department of Laboratory Medicine, Leuven, Belgium, ${ }^{2}$ Clinical Department of Laboratory Medicine, Ghent University Hospital, Gent, Belgium, ${ }^{3}$ Clinical Department of Pneumology, University Hospitals Leuven, Leuven, Belgium, ${ }^{4}$ Department of Respiratory Medicine, Ghent University Hospital, Ghent, Belgium, ${ }^{5}$ Environment and Health, Department of Public Health and Primary Care, KU Leuven, Leuven, BosniaHerzegovina

\section{Objective}

Half-life and time to seronegativity is significantly longer for anti-spike (S) than anti-nucleocapsid (N) antibodies in healthcare workers [1], but there are hardly data in hospitalized COVID-19 patients. In this study we wanted to investigate the long-term antibody response following SARS-CoV-2 infection in non-severe and severe COVID-19 patients.

\section{Material and Methods}

We retrospectively compared the long-term evolution of IgG anti-N and anti-S in 231 RT-PCR-confirmed SARS-CoV-2 infected patients from 2 university hospitals up to 365 days post positive RT-PCR. Antibodies were measured using Abbott Architect immunoassays. The study population consisted of 116 non-severe and 115 severe $(\mathrm{PaO} 2<93 \%$ and/or mechanical ventilation) patients.

\section{Results}

Peak antibody level/cut-off ratio 0-59 days after positive RT-PCR was more than 70 for anti-S compared to less than 6 for anti-N ( $\mathrm{p}<0.01)$. Anti-S and anti-N were significantly higher in severe compared to non-severe patients up to 180239 days and 300-365 days, respectively ( $\mathrm{p}<0.05$ ). The computed mean half-life for anti- $\mathrm{N}$ was 115.9 days [99.2-139.2] 
in non-severe compared to 116.3 days [101.8-135.8] in severe patients ( $\mathrm{p}=\mathrm{ns})$, with an estimated $50 \%$ of patients becoming seronegative after 273.1 and 327.3 days, respectively. For anti-S, the computed mean half-life was 141.6 days [105.7-214.6] in non-severe and 113.7 days [89.0-157.4] in severe patients ( $\mathrm{p}=\mathrm{ns}$ ), with an estimated 50\% seronegativity after 809.6 and 785.9 days, respectively.

\title{
Conclusion
}

IgG anti-S and anti-N antibody levels decay exponentially up to 365 days after a peak 0-59 days after positive RT-PCR. Estimated time to $50 \%$ seronegativity was more than 2 years for anti-S compared to less than 1 year for anti-N in nonsevere and severe COVID-19 patients due to the higher peak antibodylevel/cut-off ratio for anti-S compared to anti-N.

\section{Reference}

[1] Van Elslande J, Gruwier L, Godderis L, Vermeersch P. Estimated half-life of SARS-CoV-2 anti-spike antibodies more than double the half-life of anti-nucleocapsid antibodies in healthcare workers. Clin Infect Dis. 2021

\section{M6}

\section{IGG ANTI-SPIKE ANTIBODY LEVELS IN HEALTHCARE WORKERS WITH AND WITHOUT PRIOR COVID-19 UP TO 3 MONTHS AFTER BNT162B2 VACCINATION}

\author{
$\underline{\text { J. van Elslande }^{1}}$, M. Weemaes ${ }^{1}$, L. Godderis ${ }^{2}$, G. van Pottelbergh ${ }^{3}$, X. Bossuyt ${ }^{1}$, P. Vermeersch ${ }^{1}$ \\ ${ }^{1}$ University Hospitals Leuven, Clinical Department of Laboratory Medicine, Leuven, Belgium, ${ }^{2}$ Environment and Health, \\ Department of Public Health and Primary Care, KU Leuven, Leuven, Bosnia-Herzegovina, ${ }^{3}$ Academic Centre of General \\ Practice/Department of Public Health and Primary Care, Leuven, Belgium
}

\section{Objectives}

To study the IgG anti-spike (S) response up to 3 months after vaccination with 2 doses of BNT162b2 mRNA vaccine in healthcare workers (HCW) and compare the response to natural infection.

\section{Materials and methods}

HCW with ( $\mathrm{n}=24)$ and without ( $\mathrm{n}=126)$ a prior SARS-CoV-2 infection were sampled at baseline, 3 weeks after the first dose (20-22 days), 3 weeks after the second dose (40-45 days), and 3 months (89-105 days) after the first dose. Results were compared with HCW who had a natural SARS-CoV-2 infection and hospitalized COVID-19 patients. IgG anti-S levels were measured using Abbott Architect.

\section{Results}

All vaccinated HCW tested positive for anti-S antibodies at 6 weeks. Anti-S levels were significantly higher in HCW with prior COVID-19 compared to COVID-19 naive HCW up to 3 months after vaccination ( $\mathrm{p}<0.001)$. Anti-S titers decreased significantly faster between 6 weeks and 3 months in vaccinated COVID-19 naive HCW compared to HCW with prior COVID-19 (-66.2\% vs. $-46.7 \%, \mathrm{p}<0.001)$. At 3 months, the proportion of participants with high anti-S levels ( $\geq 4160 \mathrm{AU} / \mathrm{mL})$, suggestive of a high neutralizing antibody titer, was significantly higher in vaccinated HCW with compared to HCW without prior COVID-19 (91\% vs. 32\%, p<0.001). For comparison, only 3 of the 112 HCW (4\%) and 23 of 42 hospitalized COVID-19 patients (55\%) had a high titer 3 months after a natural infection ( $\mathrm{p}<0.001$ vs. vaccinated HCW for both).

\section{Conclusions}

Antibody response after vaccination was stronger in HCW with prior COVID-19 compared to COVID-19 naive HCW up to 3 months after the first dose. 
M7

\title{
EXPLORING THE ENDOMETRIAL MICROBIOME FOR THE FIRST TIME EVER WITH CULTUROMICS AND WASPLAB
}

\author{
$\underline{\text { R. Vanstokstraeten }}{ }^{1}$, S. Mackens ${ }^{2}$, T. Demuyser ${ }^{1}$, E. Callewaert ${ }^{1}$, K. Emmerechts ${ }^{1}$, F. Crombé ${ }^{1}$, I. Wybo ${ }^{1}$, K. Vandoorslaer ${ }^{1}$, C. \\ Blockeel $^{2}$, D. Piérard ${ }^{1}$
}

${ }^{1}$ Department of Microbiology and Infection Control, Vrije Universiteit Brussel (VUB), Universitair Ziekenhuis Brussel (UZ Brussel), Brussels, Belgium, Brussels, Belgium, ${ }^{2}$ Centre for Reproductive Medicine, Vrije Universiteit Brussel (VUB), Universitair Ziekenhuis Brussel (UZ Brussel), Brussels, Belgium, Brussels, Belgium

\section{Objective}

A possible link between microbiota and fertility has been the subject of debate since a couple of years. To date, all endometrial microbiome studies are based on metagenomics: a molecular technique that has its shortcomings in exploring low-biomass microbiomes. We have analyzed the endometrial microbiome for the first time ever using culturomics: a high-throughput culturing approach that uses multiple culture conditions and matrix-assisted laser desorption/ionization-time of flight (MALDI-TOF). We used WASPLab in order to increase traceability and reproducibility and to decrease workload.

\section{Material and methods}

Ten subfertile women undergoing diagnostic hysteroscopy as part of their routine work-up at the Centre for Reproductive Medicine were included in this study. Two different blood culture bottles with sterile rumen fluid, sterile defibrinated sheep blood and a homemade supplement mix were used for pre-incubation of the biopsies. After pre-incubation, three different anaerobe and four aerobe agar plates were inoculated. Inoculation on the agar plates of the pre-incubated samples in blood culture bottles were performed for one month on different incubation days: 1, 5, 10 and 30. A WASPLabsystem was used to process the samples.

\section{Results}

A total of 2933 colonies were identified using the MALDI Biotyper® system enabling us to identify 82 different bacterial and two different fungal species from 41 different genera, 29 different families and seven different phyla. Twenty-five of these 82 bacterial species (30\%) were Gram-negative and 33 (40\%) obligate anaerobes. The vast majority of species were for the first time ever cultured from the endometrium. Five species were for the first time ever described in the uterine microbiome: Ralstonia insidiosa, Bacillus licheniformis, Bacillus infantis, Dermacoccus nishinomiyaensis and Actinomyces radingae. Twenty-four colonies were unidentifiable and were stored in our freezer for later identification through $16 \mathrm{~S}$ rRNA gene sequencing.

\section{Conclusions}

This study highlights the potential of culturomics as a complementary technique besides metagenomics in exploring low biomass microbiomes, such as the endometrial microbiome. We also showed the utility of WASPLab in order to guarantee perfect traceability and reproducibility and to reduce the high workload of culturomics. 


\title{
M8
}

\section{PERFORMANCE OF THE ACCELERATE PHENO ${ }^{\text {TM }}$ BC KIT FOR THE MICROBIOLOGICAL DIAGNOSTIC OF BLOODSTREAM INFECTIONS CAUSED BY GRAM-NEGATIVE BACTERIA}

\author{
C. Debuysschere ${ }^{1}$, N. Sanou ${ }^{1}$, M. Potvin ${ }^{1}$, D. Martiny ${ }^{1}$ \\ ${ }^{1}$ LHUB-ULB, Bruxelles, Belgium
}

\section{Introduction}

In case of sepsis, providing fast and accurate microbiological diagnostic is crucial for the clinical management of the patient.

The Accelerate Pheno ${ }^{\mathrm{TM}}$ system is an innovative device that delivers identification (ID) and antimicrobial susceptibility testing (AST) results from a positive blood culture broth within 7 hours using a single-combined cartridge. Identifications and minimum inhibitory concentrations (MICs) are determined by FISH and morphocinetic cellular analysis, respectively.

The aim of our study is to compare, for Gram-negative isolates, the performance of the Accelerate Pheno ${ }^{\mathrm{TM}} \mathrm{BC}$ kit with our standard lab procedures in terms of identification, SIR determination and time-to-result.

\section{Material \& method}

A selection of positive blood cultures were analyzed in parallel by both the Accelerate Pheno ${ }^{\mathrm{TM}} \mathrm{BC}$ kit and our standard lab procedures. The ID by MALDI-TOF MS was considered as the gold standard and, in case of MIC discrepancies, microdilution panels and/or antimicrobial gradient strips were performed. AST performance were interpreted using Cumitech recommendations.

\section{Results}

A total of 33 samples were tested, five of which were excluded due to technical errors. Given that one strain was misidentified, the Accelerate Pheno ${ }^{\mathrm{TM}} \mathrm{BC}$ kit showed an accuracy rate of $96 \%$. As announced by the manufacturer, all IDs were provided in 1.30 hour. Regarding AST results- all provided within 7 hours- the overall categorical agreement was $97 \%$. A high rate of VME was observed (14\%), mainly related to tobramycin, ceftolozane-tazobactam, ceftazidime, aztreonam, cefuroxime and ceftriaxone. It can be explained by a low rate of resistant strains in the studied panel. Piperacillinetazobactam also presented a high rate of $\mathrm{mE}(13 \%)$.

\section{Conclusion}

The Accelerate Pheno ${ }^{\mathrm{TM}} \mathrm{BC}$ kit appears as a promising tool for the fast delivering of AST results. Results should however be interpreted with caution, particularly for ceftazidime and piperacilline-tazobactam. Further studies including a larger number of resistant strains are awaited.

\section{M9}

\section{PITFALLS OF SARS-COV-2 ANTIGEN TESTING AT EMERGENCY DEPARTMENT}

\author{
E. Cottone $^{1}$, F. van Hoecke ${ }^{1}$, G. A. Martens ${ }^{1}$, E. De Laere ${ }^{1}$, R. De Smedt ${ }^{1}$, S. Vervaeke ${ }^{1}$, M. van Hee ${ }^{1}$, D. De Smet ${ }^{1}$
}

\author{
${ }^{1}$ AZ Delta Hospital, Roeselare, Belgium
}

Current method for diagnosis of SARS-CoV-2 infection is RT-PCR test on nasopharyngeal or oropharyngeal swab. Rapid diagnosis is essential for containing viral spread and triage of symptomatic patients presenting to hospital ER departments. As a faster alternative to RT-PCR, rapid antigen tests became available using LFA. Our study showed 
inferior performance of the Roche SARS-Cov-2 Rapid Antigen test (SD Biosensor) for detection of SARS-CoV-2 which limits its use as a diagnostic gatekeeper at ER-departments. Firstly, because of a lack of specificity, which is potentially life threatening due to admission to a COVID-19 cohort-department and the associated high risk of nosocomialacquired SARS-CoV-2 infection. Secondly, with a sensitivity of $45,5 \%$ it is not possible to confidently rule out SARS-CoV-2 infection, resulting in the compelling need for reflex PCR. Comparison of viral load in RT-PCR positive samples with corresponding antigen results, showed a significant difference between antigen positive and antigen negative samples. COVID-19 infection will not be detected in patients admitting to the hospital in an early or late phase, typically associated with low viral loads. Sensitivity increases significantly when testing within 5-7 symptomatic days, probably due to higher viral load, but patients often admit to an ER-department after a longer symptomatic period, making a cutoff of 5-7 symptomatic days for use of antigen tests impractical in ER-triage. However, differentiation between contagious and non-contagious individuals may be possible, as diagnostic performance is better to detect high viral load $(>=5 \log 10 \mathrm{copies} / \mathrm{mL}$ ) linked with contagiousness. This opens up possibilities for events or gatherings.

\title{
M10
}

\section{COVID-19 ASSOCIATED PULMONARY ASPERGILLOSIS: INCIDENCE AND DIAGNOSIS IN THE INTENSIVE CARE UNIT POPULATION OF A TERTIARY CARE HOSPITAL}

\author{
$\underline{\text { M. Vanrenterghem }}^{1}$, M. Reynders ${ }^{2}$ \\ ${ }^{1}$ University of Leuven, Leuven, Belgium, ${ }^{2}$ Algemeen Ziekenhuis Sint-Jan Brugge-Oostende, Brugge, Belgium
}

\section{Objective}

COVID-19 associated pulmonary aspergillosis (CAPA) has been documented as an emerging complication in intensive care unit (ICU) patients. The prevalence and mortality ratio was investigated in hospitalized COVID-19+ ICU patients.

\section{Materials and methods}

Between February 2020 and April 2021, we conducted a retrospective analysis in critically ill COVID-19 patients, who had a bronchoalveolar lavage fluid (BAL) with GM-EIA and PCR Aspergillus fumigatus measurements.

\section{Results}

In total, 40 COVID-19 patients, who were mechanical ventilated, were included. Of these patients 17,5\% (7/40) tested positive for GM (ODI >1.0) on BAL. All patients had concordant results with the Aspergillus fumigatus PCR and cycle thresholds (Ct) varied between 26.91 and 35.0, suggestive for significant positive results. All patients were treated with voriconazole. A positive GM-EIA in endotracheal aspirates was a preceding trigger for performing a bronchoscopy with BAL. Only two patients had pre-existing bronchial disease (astma and COPD GOLD IV). Others had no identified preexisting risk factor for IPA. The 90-day mortality ratio was 43\% (4/7).

\section{Conclusion}

We found a relative high prevalence of CAPA in this investigated study group with a high mortality ratio. Local awareness should be raised for CAPA in critically ill COVID-19 patients. We recommend screening mechanically ventilated patients with galactomannan on high respiratory samples (using a cut-off of at least 1.0 to 2.0), to facilitate early detection. 


\title{
M11
}

\section{IMPACT OF « ALFRED 60AST » ON THE CLINICAL MANAGEMENT OF PATIENTS SUFFERING FROM ENTEROBACTERIAL SEPSIS}

\author{
$\underline{\text { M. Potvin }^{1}}$, N. Sanou ${ }^{1}$, M. Muys ${ }^{1}$, F. De Bièvre ${ }^{2}$, G. Verschelden ${ }^{2}$, E. Larranaga Lapique ${ }^{2}$, M. Hites ${ }^{2}$, D. Martiny ${ }^{1}$ \\ ${ }^{1}$ Microbiology Department, LHUB-ULB, Brussels, Belgium, ${ }^{2}$ Department of Infectious Disease, Erasme Hospital, Brussels, \\ Belgium

\section{Introduction}

Sepsis is an important cause of morbidity and mortality. An accelerated microbiology diagnosis is crucial in order to reduce the time to initiate targeted antibiotic therapy. The Alfred $60^{\mathrm{AST}}$ system is able to provide phenotypic Antimicrobial Susceptibility Testing (AST) results within three to five hours. This study has two objectives. First, to assess the impact of this new technology on the therapeutic management of patients with bloodstream infections. Second, to determine the cost-effectiveness of its use and its impact on the workflow of a clinical microbiology laboratory.

\section{Material \& Methods}

During a ten weeks period, all new episode of bloodstream infection caused by Enterobacteriaceae (based on Gram staining), and occurring at night or in the morning, were analyzed by the Alfred $60^{\mathrm{AST}}$ system in parallel with our routine methods. The time-to-result (TTR) and the impact of the rapid AST on the clinician's therapeutic strategy were studied. In order to assess the financial and practical aspects of the method, an analysis of the hidden costs and a survey involving the technical staff were conducted.

\section{Results}

Forty-two blood samples were included. A significant reduction of the TTR ( $13 \mathrm{~h} 25 \mathrm{vs} 32 \mathrm{~h} 25$; $\mathrm{p}<0,001)$ was demonstrated and $18,9 \%$ of the rapid AST results led to a modification of the therapeutic strategy. The financial analysis highlighted an increase in costs of almost $40 \%$ compared to the theoretical costs reported by the manufacturer, mainly due to technical issues and short expiry date of the reactants. Finally, results of the technical staff survey were favorable in terms of ease of use of the system.

\section{Conclusion}

In addition to its ease of use, the Alfred60 ${ }^{\mathrm{AST}}$ system is able to provide an AST in a record time. This study shows a real interest in this system for the therapeutic management of patients affected by enterobacterial sepsis. However, its implementation requires an increase of the sample volumes and a 24/7 organization of the laboratory in order to be profitable.

\section{M12}

\section{MICROBIOLOGICAL AND CLINICAL FEATURES OF HELICOBACTER CANIS REPORTS}

\author{
$\underline{\text { B. Lardinois }}{ }^{1}$, L. Belkhir ${ }^{2}$, A. Verroken ${ }^{1}$
}

${ }^{1}$ Department of Microbiology, Cliniques universitaires Saint Luc, Université Catholique de Louvain, Brussels, Belgium., Bruxelles, Belgium, ${ }^{2}$ Department of Internal Medicine and Infectious Diseases, Cliniques universitaires Saint Luc, Université Catholique de Louvain, Brussels, Belgium, Bruxelles, Belgium

\section{Background}

Helicobacter canis, an enterohepatic Helicobacter has proven its role in human diseases and has been rediscussed in recent years as its zoonotic potential is increasingly described. Routine microbiological detection of this pathogen is a difficult task as its culture may fail due to fastidious growth. It is therefore supposed that many clinical laboratories under-recognize $H$. canis infections. 


\section{Objectives}

Determine current microbiological trends of $H$. canis, its susceptibility profile and its frame of infectious diseases for a better understanding of the pathogen by clinicians and microbiologists.

\section{Material and Methods}

We reviewed all clinical and microbiological litterature currently available from previous relevant $H$. canis human clinical cases, mainly bacteremias, and added the data of a clinical case observed at the Cliniques universitaires Saint-Luc.

\section{Results}

Clinical features of $H$. canis reports are summarized in Table 1 . The observed microbiological trends from 10 cases of bacteremias were a median of 4 days (min-Max: 2-6) until positive aerobic blood culture bottle detection, subcultures showing a thin layer of small colonies on enriched non-selective media under microaerophilic atmosphere at $35-42^{\circ} \mathrm{C}$ after 3 to 4 days of growing then an identification requiring 16S rRNA sequencing given the difficulties observed with MALDI-TOF MS. Low MICs were observed for penicillins, amoxicillin/clavulanic acid, carbapenems and metronidazole in opposite to high MICs for ciprofloxacin.

Table 1: Clinical features of Helicobacter canis reports

\begin{tabular}{|c|c|c|}
\hline Variables ${ }^{\mathrm{a}}$ & $\begin{array}{l}\text { H. canis reports } \\
\mathrm{N}=12\end{array}$ & $\%$ or min-max \\
\hline \multicolumn{3}{|l|}{ Demographics } \\
\hline Adults & 9 & 75 \\
\hline Female & 6 & 50 \\
\hline Age (years) & 46.5 & $0.2-78.0$ \\
\hline Underlying conditions in adults $(\mathrm{N}=9)$ & 8 & 89 \\
\hline Immunosuppressors & 5 & 56 \\
\hline ESRD & 3 & 33 \\
\hline Splenectomized & 2 & 22 \\
\hline Qthers & 5 & 56 \\
\hline \multicolumn{3}{|l|}{ Clinical findings } \\
\hline Fever & 7 & 58 \\
\hline Skin abnormalities & 4 & 33 \\
\hline Digestive symptoms & 4 & 33 \\
\hline \multicolumn{3}{|l|}{ Laboratory findings } \\
\hline Bacteraemia & 10 & 83 \\
\hline WBC at hospitalization $\left(\times 10^{9} / \mathrm{L}\right)$ & 10.350 & $7.900-15.000$ \\
\hline CRP at hospitalization $(\mathrm{mg} / \mathrm{dL})$ & 7.7 & $0-9.4$ \\
\hline Close contact with pets & $10^{c}$ & 83 \\
\hline Dog & 8 & 67 \\
\hline Cat & 4 & 33 \\
\hline \multicolumn{3}{|l|}{ Management and outcome } \\
\hline Cephalosporin based antibiotherapy & 7 & 58 \\
\hline In-hospital time (days) & 5 & $3-15$ \\
\hline Fatal outcome & $1^{\mathrm{d}}$ & 8 \\
\hline
\end{tabular}

${ }^{a}$ Variables expressed as percentage of cases (\%) or median (minimum-maximum observed rank)

${ }^{b}$ Chronic pancreatitis, lymphoma, sarcoidosis, Crohn's disease or theumatoid arthritis

'Two cases did not mention the presence or absence of a pet

${ }^{d}$ One case reported $H$. canis bacteremia in a two-month infant with cardiorespiratory arrest

CRP, C-reactive protein; ESRD, End-stage renal disease; WBC, White blood cells 


\title{
Conclusions
}

We identified a frequent association of $H$. canis with bacteremia in immunocompromised patients with recurrent fever in contact with pets, especially dogs. Considering the fastidious growing capacities, definitive identification from blood cultures may not be expected before 7 days. Intravenous ceftriaxone, oral doxycycline or metronidazole have been suggested as efficient therapeutic choices.

\section{H1}

\section{SCREENING FOR MICROCYTIC ANAEMIAS: A NEW ALGORITHM USING SYSMEX XN-9000 SPECIFIC ERYTHROCYTE AND RETICULOCYTE PARAMETERS}

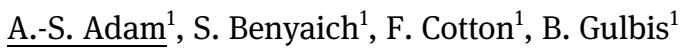 \\ ${ }^{1}$ LHUB-ULB, Clinical Chemistry, Brussels, Belgium

\section{Background}

Anaemia is a global public health problem that affects almost one third of the world population. Thalassaemia and iron deficiency anaemia are the most common causes of microcytic anaemia, with similar clinical and laboratory features, making their diagnostic often complicated. During the last decade, cell counter-based formulae and algorithms have been proposed in the differential diagnosis of microcytic anaemia. Unfortunately, only a few studies consider certain not so rare clinical situations where microcytic anaemia may occur.

\section{Objectives}

To develop an algorithm as first tier screening tool in the differential diagnosis of microcytic anaemias.

\section{Methods}

We retrospectively analyzed results of complete red blood cells (RBCs) and reticulocyte counts obtained on the Sysmex XN-9000 of 280 adults, divided into 5 groups: iron deficiency anaemia (IDA, $n=56$ ), $\beta$-thalassaemia minor (BTM, $n=68$ ), microcytic heterozygous haemoglobinopathies (HTZHB, $n=24$ ), hereditary spherocytosis (HS, n=14) and patients with no suspicion of IDA or hereditary RBCs disorders (OTHERS, $\mathrm{n}=118$ ). A Classification and Regression Tree (CART) analysis was performed to obtain an algorithm in order to predict these groups.

\section{Results}

Four parameters were selected: the percentage of microcytic RBCs (\%MicroR), the \%MicroR to percentage of hypochromic RBCs ratio (\%MicroR/\%Hypo-He), the reticulocyte haemoglobin content (RET-He) and the reticulocyte count to immature reticulocyte fraction ratio (RET/IRF) (Figure 1). When applying the algorithm, 93/106 of the hereditary RBC disorder patients and 41/56 IDA patients were corectly screened. The correct overall classification rate reached $83.6 \%$. The best sensitivity and specificity were obtain for the screening of BTM, with $92.6 \%$ and $94.8 \%$ respectively.

\section{Conclusion}

We proposed a costless, affordable, easy to use and effective algorithm that can be used routinely for the differential diagnosis of microcytic anaemias. The latter is based on erythrocyte and reticulocyte parameters offered by the most recent generation of the Sysmex analyzers, the XN-9000.

\section{References}

1. Nivaggioni, V., et al., Use of Sysmex XN-10 red blood cell parameters for screening of hereditary red blood cell diseases and iron deficiency anaemia. Int J Lab Hematol, 2020. 42(6): p. 697-704.

2. Mullier, F., et al., Additional erythrocytic and reticulocytic parameters helpful for diagnosis of hereditary spherocytosis: results of a multicentre study. Ann Hematol, 2011. 90(7): p. 759-68. 


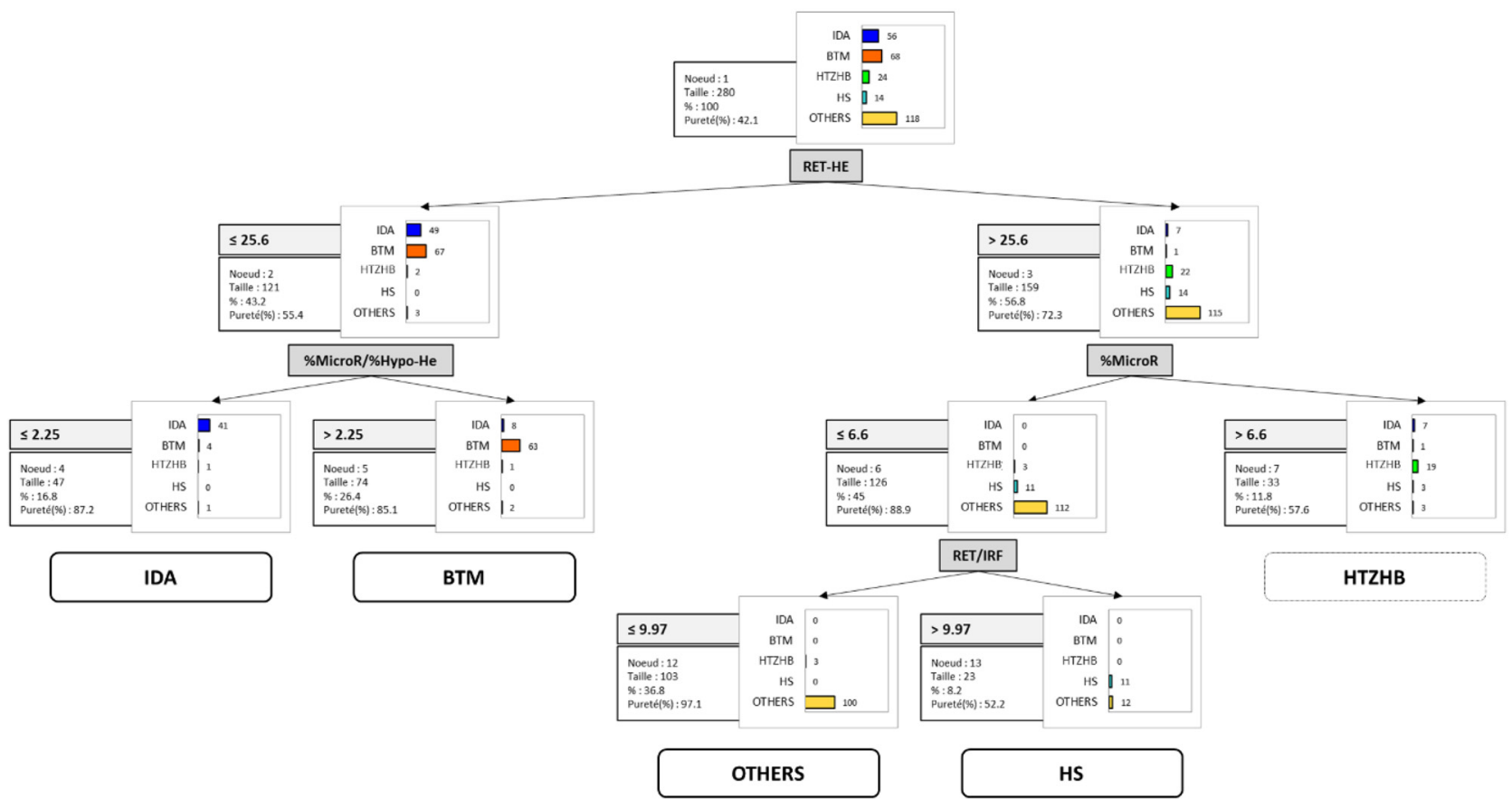

Figure 1: Algorithm for the screening of microcytic anaemias obtained by the CART analysis.

3. Bobée, V., et al., Screening of hereditary spherocytosis and pyruvate kinase deficiency by automated blood count using erythrocytic and reticulocytic parameters. Int J Lab Hematol, 2018. 40(6): p. 697-703.

4. Urrechaga, E., L. Borque, and J.F. Escanero, Erythrocyte and reticulocyte parameters in iron deficiency and thalassemia. J Clin Lab Anal, 2011. 25(3): p. 223-8.

\title{
H2
}

\section{EVALUATION OF RET-HE FOR THE DETECTION OF MARTIAL DEFICIENCY IN PREOPERATIVE}

\author{
O. D. M. De Mos ${ }^{1}$, G. M. Mathy ${ }^{1}$, A. G. Gothot ${ }^{2}$
}

${ }^{1}$ Laboratoire de Biologie Clinique, CHR Verviers, Liège, Belgium, ${ }^{2}$ Service d'Hématologie biologie et ImmunoHématologie, CHU de Liège, Liège, Belgium

\section{Objective}

Recent technological advances in flow cytometry have made it possible to measure the hemoglobin content of reticulocytes (RET-He - Sysmex®). RET-He is an inexpensive, rapid and easily automated parameter that highlights the actual iron contribution to erythropoiesis and allows to judge the quality of red blood cells. Numerous studies have demonstrated its value in the diagnosis of iron deficiency and in the monitoring of iron supplementation. This study investigates the value of RET-He in the context of preoperative screening for iron deficiency to facilitate its management.

\section{Material and Methods}

Prospectively, from February 1 to March 31, 2021, samples of 138 patients coming from the anesthesia consultation of the hospital of the CHR of Verviers were collected and analyzed. Iron status was defined according to an algorithm for the management of preoperative martial deficiency recommended by the "Groupe francophone de Réhabilitation Améliorée après Chirurgie”. The diagnostic performance and the optimal threshold of the RET-He could be determined. 


\title{
Results
}

The RET-He correlated significantly with the usual parameters of martial deficiency such as iron, ferritin and transferrin saturation coefficient. The sensitivity of the RET-He is $58.3 \%$ and the specificity is $97.3 \%$ with an overall efficiency of $90.6 \%$. With a threshold set at $32.8 \mathrm{pg}$, the RET-He has a negative predictive value of $97.8 \%$. These results demonstrate that the RET-He is quite efficient in predicting the absence of martial deficiency.

\section{Conclusion}

In addition to its low cost and ease of use, the RET-He appears to be particularly effective in excluding the presence of martial deficiency in the general population. With a threshold of $32.8 \mathrm{pg}$, its negative predictive value is $97.8 \%$. The RET-He could be an important parameter in a "rule out" algorithm for martial deficiency used in anaesthesia consultations. The current priority is to make clinicians aware of the need for systematic screening and management of martial deficiency preoperatively. The use of the RET-He could be proposed in the anesthesia consultation to simplify this screening.

\section{H3}

\section{EVALUATION OF THE SEBIA CAPILLARY ELECTROPHORESIS (CAPILLARYS III) FOR HEMOGLOBINOPATHY TESTING}

\author{
$\underline{\text { S. Cherkaoui }}{ }^{1}$, N. Tasiaux ${ }^{1}$, M. De Laveleye ${ }^{2}$, C. Chevalier ${ }^{1}$
}

${ }^{1}$ Department of Clinical Biology, Sector of Hematology, CHU Charleroi, Lodelinsart, Belgium, Charleroi, Belgium, ${ }^{2}$ Department of Clinical Biology, Sector of Clinical Chemistry, CHU Charleroi, Lodelinsart, Belgium, Charleroi, Belgium

\section{Introduction}

The term hemoglobinopathy describes both quantitative (thalassemias) and qualitative (variants) hemoglobin ( $\mathrm{Hb})$ disorders. High-pressure liquid chromatography (HPLC) and capillary zone electrophoresis (CZE) are the most widely used methods for the diagnosis of hemoglobinopathies. The Capillarys system (Sebia, Lisses, France) uses the principle of CZE in free solution.

\section{Goal}

The aim of this study was to evaluate the performance of the Capillarys III (Sebia, Lisses, France) to compare the performance of the Capilalrys II (Sebia, Lisses, France) routinely used at the CHUCharleroi for the analysis of qualitative and quantitative hemoglobin abnormalities.

\section{Material and methods}

Capillary electrophoresis of $\mathrm{Hb}$ by Capillarys III ${ }^{\circledR}$ is done on total blood (K2EDTA) using a 'cap piercing' system. The electrophoretic profiles are analyzed on the Phoresis ${ }^{\circledR}$ software

- Correlation:

75 adult samples and 30 cord bloods from the routine of the CHUCharleroi were analysed in parallel on Capillarys II and III. A Bland-Altmann and a Passing-Bablok correlation was made with Medcalc version 19 (Ostend, Belgium). Samples with abnormal $\mathrm{Hb}$ fractions were also analyzed by HPLC on the Tosoh G8 (Tosoh Europe)

- Accuracy:

Within-run precision was determined by analyzing 7 samples with $\mathrm{Hb}$ variants (Hb AS, SS, CC, AC, F, Lepore) in 20 replicates. The same samples were analyzed once a day for 10 days for the between-run precision allowing the calculation of means, standard deviations (SD) and coefficients of variation (CV) for each $\mathrm{Hb}$ fraction. 
- Inaccuracy:

Evaluated by comparing our values to the ones obtained on external quality controls (UK NEQAS).

\title{
Results
}

The Capillarys III can recognize both normal and abnormal $\mathrm{Hb}$ peaks and shows a perfect agreement with the TOSOH.

The Passing-Bablok and Bland-Altman analysis show a good correlation between Capillarys II and III, for all hemoglobin fractions.The within and between run precision study results showed acceptable variability (CVs $<1 \%)$.

The measurement uncertainty obtained for $\mathrm{Hb} \mathrm{A} 2, \mathrm{Hb} \mathrm{F}$ and $\mathrm{Hb} \mathrm{S}$ is very satisfactory for each of these three fractions (bias for $\mathrm{Hb} \mathrm{A} 2=9.92 \%, \mathrm{Hb} \mathrm{F}=8.33 \%$ and $\mathrm{Hb} \mathrm{S}=0.51 \%)$.

\section{Conclusion}

The Capillarys III system can separate and measure all important $\mathrm{Hb}$ fractions in adult and cord blood. It gives good analytical performance and shows a good correlation with the Capillarys II and the TOSOH. It is easy to use and allows a rate of analysis quite satisfactory for a routine analysis.

H4

\section{HIGHLIGHTING AND IDENTIFICATION OF RHD VARIANT ALLELES IN PREGNANT WOMEN DURING THE FETAL RHD GENOTYPING}

\author{
P. Dufour ${ }^{1}$, C. Gerard ${ }^{2}$, F. Chantraine ${ }^{1}$, J.-M. Minon ${ }^{1}$ \\ ${ }^{1} \mathrm{CHR}$ Citadelle Liège, Liège, Belgium, ${ }^{2} \mathrm{CHU}$ Liège University of Liège, Liège, Belgium
}

\section{Objectives}

We developed an algorithm allowing solving discordant results in fetal $R H D$ genotyping assay. The aim of the algorithm was to highlight and identify the maternal $R H D$ variant alleles. The present work reports the diagnostic efficiency of this algorithm applied for 14 years.

\section{Material and methods}

Between 2006 and 2020, 15690 fetal RHD genotyping assays were performed. When isolated amplification of 1 or 2 exons and/ or low Ct were observed during fetal $R H D$ genotyping, presence of $R H D$ variant alleles was researched in maternal buffy coat by real-time PCR. And when PCR was positive, the variant was identified by sequence specific primer PCR.

\section{Results}

A RHD variant allele was highlighted in 247 women: 68 women presented weak variants (among which 45 weak type 1,2 or 3), 9 partial weak variants, 24 partial variants, 2 DEL variants, 117 silent variants while 26 RHD variant could not be identified. In addition, a new variant was identified and sequenced (presence of an $883 \mathrm{~A}>\mathrm{G}$ substitution).

\section{Conclusions}

This algorithm, which includes the demonstration of a variant maternal allele, presents several clinical advantages: when the maternal variant is associated with an isolated amplification of exon 1 or 2 , the fetal RHD can still be determined as a function of the other exons. In addition, women with weak $\mathrm{RhD}$ type 1, 2 or 3 can safely be considered RhD positive for either transfusion or immunoprophylaxis. This algorithm, which increases the quality of pregnancy monitoring, also contributes to the sparing of RhD negative blood components. 


\title{
H5
}

\section{LABORATORY DIVERGENCES IN CONCURRENT DIAGNOSIS OF ACUTE MYELOID LEUKEMIA RELAPSE AND COVID-19: A CASE REPORT}

\author{
A. Nizet $^{1}$, J. Foguenne ${ }^{1}$, A. Gothot ${ }^{1}$, F. Tassin ${ }^{1}$, A. Keutgens ${ }^{1}$ \\ ${ }^{1}$ Department of Laboratory Hematology, University Hospital of Liege, University of Liege, Liege, Belgium
}

Objectives

Acute myeloid leukemias (AML) are well-known and are not rarely reported. However, in time of coronavirus disease 2019 (COVID-19) pandemic, diagnosis, follow-up and care of patients with AML can become a huge challenge for clinicians and clinical laboratory specialists, due to a lack of experience and the presence of divergences in both clinical examination and laboratory results.

Here, we reported the admission in the emergency department of a 61-year-old patient in complete remission for 6 years for an AML, with a flu syndrome, productive cough, headaches, anosmia and ageusia.

\section{Material and methods}

At the admission, physical examination, chest computed-tomography (CT) scan, nasopharyngeal swab, blood test and bone marrow (BM) aspirate were performed due to both symptoms of COVID-19 and the patient's AML history. SARS-CoV2 PCR was realized in the nasopharyngeal swab. The cytology of blood cells, in the peripheral blood and in the BM, were observed under a microscope to highlight any abnormalities. Flow cytometry immunophenotypic analysis was used in addition to bone marrow cytology.

\section{Results}

Physical examination and CT-scan revealed no abnormalities suggestive of SARS-CoV-2 infection. Blood tests showed a mild inflammatory syndrome and a pancytopenia. Peripheral blood smear demonstrated the presence of multiple plasma cells (5.5\%) and activated lymphocytes (4.5\%), whereas BM aspirate smear revealed mild lymphocytosis with few activated lymphocytes and some plasma cells (approximatively 5\%), as previously noticed in the peripheral blood sample. Otherwise, a large blast population (approximatively 24\%) was visualized in the BM and confirmed by flow cytometry immunophenotypic analysis (5\% myeloblast: weak CD45, HLA-DR, CD34, CD117, strong CD33, weak CD11c, weak CD15, and CD13). Finally, SARS-CoV-2 PCR detected the presence of the virus in the nasopharyngeal swab.

\section{Conclusion}

Concurrent diagnosis of COVID-19 and AML relapse involved an unconventional care by treating two potentially lifethreatening diseases at the same time. In this case, the patient was treated firstly for SARS-CoV-2 infection, as recommended. Evolution of AML and its blood infiltration was showed four weeks after admission. Finally, patient was treated by allogeneic stem cell transplantation two months after diagnosis of the AML relapse.

\section{H6}

\section{CONTINUOUS PLATELETS TRANSFUSION FOR REFRACTORY PATIENTS}

\author{
A. Devey ${ }^{1}$, J. Schmitt', M. Binsfeld ${ }^{1}$, G. Maggipinto ${ }^{1}$, A. Gothot ${ }^{1}$ \\ ${ }^{1} \mathrm{CHU}$ de Liège, Liège, Belgium

\section{Objectives}

Patient with platelet transfusion refractoriness are quite frequent in hematology centers and keeping a platelet count allowing bleeding prevention or bleeding drying up is challenging under these conditions. One of the therapeutic 
option consist in transfusing platelets concentrates (PC) continuously, meaning transfusing slowly (in 4 to 6 hours) 3 to 4 PC over 24h.

The goal of the present study was, on the one hand, to check the absence of platelets increment, at $1 \mathrm{~h}$ et $24 \mathrm{~h}$, before starting the continuous transfusion (CT) while studying the reasons of refractoriness and, on the other hand, to analyze biological and clinical outcomes of this strategy among daily platelet count or clinical events such as new bleeding, stopped bleeding...etc.

\title{
Material \& Methods
}

For this purpose, we performed a retrospective study among CHU de Liège's inpatients from 01/01/2019 to 31/12/2020. An extraction from our software (Glims ${ }^{\circledR}$ ) and a medical records' (Omnipro ${ }^{\circledR}$ ) review of patients who received at least 3 PC in $24 \mathrm{~h}$ allowed us to select patients with CT. Refractoriness to platelet transfusion and bleeding gradation were assessed, respectively, according to the HAS definition and the WHO bleeding grade. For biological assessment, morning platelet counts were considered.

\section{Results}

At the end, 13 patients received at least 1 episode of CT of PC within the study period; for 9 of them, non-immune causes explained refractoriness. Mean platelet counts during CT were significantly $(\mathrm{p}<0.0001)$ higher than before CT. Furthermore, in $94.4 \%$ of cases, there was, at least no bleeding deterioration and, in $61 \%$ we observed an improvement of the symptoms. We also observed minor transfusion reactions (TR) whose prevalence fits our hospital's TR rate.

Conclusion With limitations due to retrospective study, we can state that continuous platelet transfusion is a safe and effective procedure used to maintain a platelet count able to protect patients from bleeding or to prevent bleeding deterioration.

H7

\section{MONITORING OF UNFRACTIONATED HEPARIN THERAPY IN THE INTENSIVE CARE UNIT USING A POINT-OF-CARE APTT: A COMPARATIVE OBSERVATIONAL STUDY WITH LABORATORY-BASED APTT AND ANTI-XA ACTIVITY MEASUREMENT}

\author{
B. Lardinois ${ }^{1}$, M. Hardy ${ }^{2}$, I. Michaux ${ }^{3}$, G. Horlait ${ }^{3}$, T. Rotens ${ }^{3}$, H. Jacqmin ${ }^{1}$, S. Lessire ${ }^{2}$, P. Bulpa ${ }^{3}$, A. Dive ${ }^{3}$, F. Mullier ${ }^{1}$
}

${ }^{1}$ Université catholique de Louvain, CHU UCL Namur, Namur Thrombosis and Hemostasis Center, Hematology Laboratory, Yvoir, Belgium, ${ }^{2}$ Université catholique de Louvain, CHU UCL Namur, Namur Thrombosis and Hemostasis Center, Anesthesiology Department, Yvoir, Belgium, ${ }^{3}$ Université catholique de Louvain, CHU UCL Namur, Namur Thrombosis and Hemostasis Center, Department of Intensive Care, Yvoir, Belgium

\section{Background}

Continuous intravenous unfractionated heparin (UFH) is administered routinely in the intensive care unit (ICU) for anticoagulation of patients and monitoring is performed by the activated partial thromboplastin time (APTT) or anti-Xa activity. However, these strategies are associated with potentially large time intervals before dose adjustments, which could be detrimental to the patient.

\section{Objectives}

To compare a point-of-care (POC) version of the APTT to (i) laboratory-based APTT and (ii) measurement of anti-Xa activity in terms of correlation, agreement and turnaround time (TAT). 


\section{Material and Methods}

35 ICU patients requiring UFH therapy were prospectively included after giving their written informed consent. Simultaneous measurements of POCT-APTT (CoaguCheck aPTT Test ${ }^{\circledR}$, Roche Diagnostics) on a drop of fresh whole blood, laboratory-based APTT (C.K. Prest ${ }^{\circledR}$, Stago) and Anti-Xa activity (STA ${ }^{\circledR}$ Liquid anti-Xa, Stago) on 109mM buffered citrated plasma were systematically performed before dose adjustment. Time tracking of sampling and analysis was realized.

\section{Results}

The overall correlation between POCT-APTT vs laboratory APTT ( $\mathrm{n}=795$ pairs) and POCT-APTT vs Anti-Xa ( $\mathrm{n}=729$ pairs) are presented in Figure 1. There were only fair agreements between POCT vs laboratory APTT (Cohen's kappa ( $\mathrm{k}) 0.27(95 \%$ CI 0.22-0.32)), and between POCT vs Anti-Xa activity ( $к 0.30$ (IC95\% 0.23-0.37)). The median TATs from sample collection to the lab delivery of results for Lab-APTT and anti-Xa were 50.9 minutes (Interquartile range (IQR), 30.6) and $66.3 \mathrm{mi}$ nutes (IQR, 42.8), respectively, while the POCT delivered results in less than 5 minutes $(\mathrm{p}<0.0001)$.

\section{Conclusion}

Although the POCT-APTT device significantly reduces time to results its use should not be recommended when using either anti-factor Xa activity or activated partial thromboplastin time (aPTT)-based nomograms.
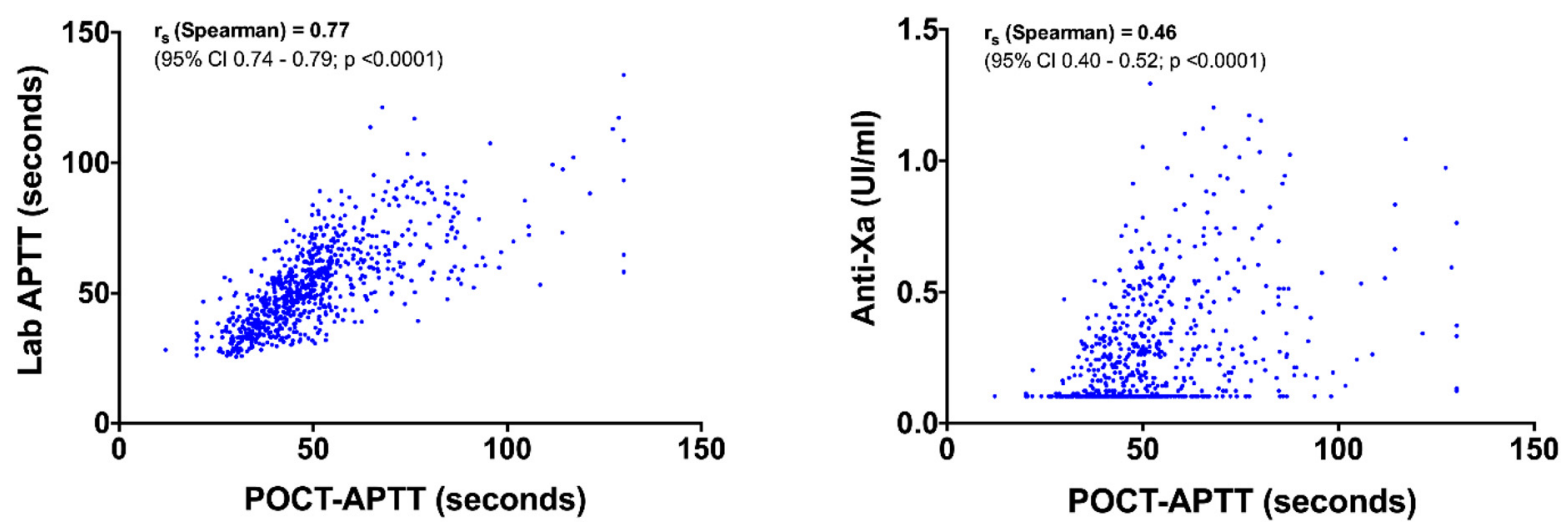

Figure 1: Overall correlation of POCT-APTT vs laboratory APTT (left panel, N=795) and POCT-APTT vs Anti-Xa (right panel, $\mathrm{N}=729$ ) from the 35 patients included in the study. 